\title{
Bilinear Hilbert Transforms and (Sub)Bilinear Maximal Functions along Convex Curves
}

\author{
Junfeng Li and Haixia Yu*
}

\begin{abstract}
In this paper, we determine the $L^{p}(\mathbb{R}) \times L^{q}(\mathbb{R}) \rightarrow L^{r}(\mathbb{R})$ boundedness of the bilinear Hilbert transform $H_{\gamma}(f, g)$ along a convex curve $\gamma$

$$
H_{\gamma}(f, g)(x):=\text { p.v. } \int_{-\infty}^{\infty} f(x-t) g(x-\gamma(t)) \frac{\mathrm{d} t}{t},
$$

where $p, q$, and $r$ satisfy $\frac{1}{p}+\frac{1}{q}=\frac{1}{r}$, and $r>\frac{1}{2}, p>1$, and $q>1$. Moreover, the same $L^{p}(\mathbb{R}) \times L^{q}(\mathbb{R}) \rightarrow L^{r}(\mathbb{R})$ boundedness property holds for the corresponding (sub)bilinear maximal function $M_{\gamma}(f, g)$ along a convex curve $\gamma$
\end{abstract}

$$
M_{\gamma}(f, g)(x):=\sup _{\varepsilon>0} \frac{1}{2 \varepsilon} \int_{-\varepsilon}^{\varepsilon}|f(x-t) g(x-\gamma(t))| \mathrm{d} t .
$$

\section{Contents}

1 Introduction 2

1.1 Main problem and main result $\ldots \ldots \ldots \ldots \ldots \ldots$

1.2 Background and motivation . . . . . . . . . . . . . 4

1.3 Organization and notations .................... 5

2 Preliminaries 7

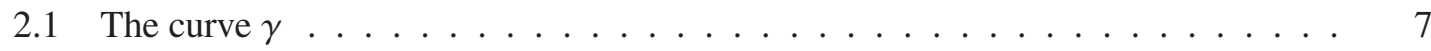

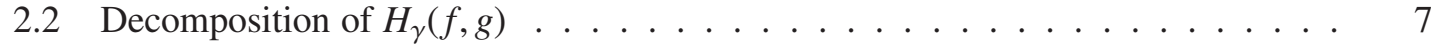

2.3 Decomposition of $M_{\gamma}(f, g) \ldots \ldots \ldots \ldots \ldots$

3 The boundedness of $H_{\gamma}^{1}(f, g)$

4 The boundedness of $H_{\gamma}^{2}(f, g)$

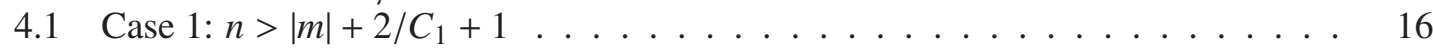

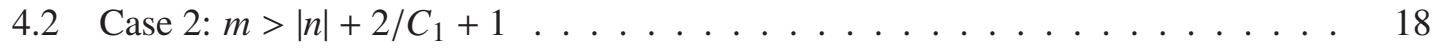

2010 Mathematics Subject Classification. Primary 42B20; Secondary 47B38.

Key words and phrases. Bilinear Hilbert transform, (sub)bilinear maximal function, convex curve, time frequency analysis.

Junfeng Li is supported by NSFC-DFG (\# 11761131002), Haixia Yu is supported by "the Fundamental Research Funds for the Central University" (\# 20lgpy144).

${ }^{*}$ Corresponding author. 
5 The $L^{2}(\mathbb{R}) \times L^{2}(\mathbb{R}) \rightarrow L^{1}(\mathbb{R})$ boundedness of $H_{\gamma}^{3}(f, g)$

5.1 An estimate by using $T T^{*}$ argument . . . . . . . . . . . . 22

5.2 Another estimate by $\sigma$-uniformity and the $T T^{*}$ argument . . . . . . . . . . 26

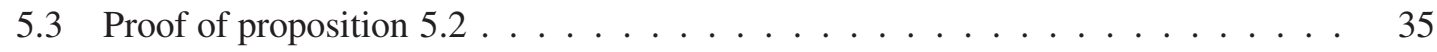

6 Weak- $L^{p}(\mathbb{R}) \times L^{q}(\mathbb{R}) \rightarrow L^{r}(\mathbb{R})$ boundedness of $H_{m}(f, g)$

$6.1 \quad$ Error terms $\left|H_{m}^{1}\right|(f, g),\left|H_{m}^{2}\right|(f, g),\left|H_{m}^{3}\right|(f, g) \ldots \ldots \ldots \ldots \ldots \ldots \ldots$

6.2 Major term $\left|H_{m}^{4}\right|(f, g) \ldots \ldots \ldots \ldots \ldots \ldots \ldots \ldots$

6.2.1 Sizes and BMO estimates . . . . . . . . . . . . . 44

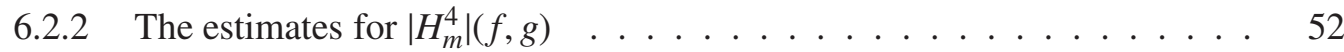

7 The boundedness of $M_{\gamma}(f, g)$

\section{Introduction}

\subsection{Main problem and main result}

The bilinear Hilbert transform $H_{\gamma}(f, g)$ along a curve $\gamma$ is defined as

$$
H_{\gamma}(f, g)(x):=\mathrm{p} . \mathrm{v} \cdot \int_{-\infty}^{\infty} f(x-t) g(x-\gamma(t)) \frac{\mathrm{d} t}{t}
$$

for $f$ and $g$ in the Schwartz class $\mathcal{S}(\mathbb{R})$. The corresponding (sub)bilinear maximal function $M_{\gamma}(f, g)$ is defined as

$$
M_{\gamma}(f, g)(x):=\sup _{\varepsilon>0} \frac{1}{2 \varepsilon} \int_{-\varepsilon}^{\varepsilon}|f(x-t) g(x-\gamma(t))| \mathrm{d} t .
$$

The $L^{p}(\mathbb{R}) \times L^{q}(\mathbb{R}) \rightarrow L^{r}(\mathbb{R})$ boundedness property for these two operators with some general curves $\gamma$ are of great interest to us. We start with a special case $\gamma:=\mathrm{P}$, a polynomial of degree $d$ with no linear term and constant term, where $d \in \mathbb{N}$ and $d>1$. In [37], Li and Xiao set up the $L^{p}(\mathbb{R}) \times L^{q}(\mathbb{R}) \rightarrow L^{r}(\mathbb{R})$ boundedness for $H_{\gamma}(f, g)$ and $M_{\gamma}(f, g)$ where $p, q$, and $r$ satisfy $\frac{1}{p}+\frac{1}{q}=\frac{1}{r}$, and $r>\frac{d-1}{d}, p>1$, and $q>1$. Moreover, they showed that $r>\frac{d-1}{d}$ is sharp up to the end point. By replacing $\gamma$ with a homogeneous curve $t^{d}$ with $d \in \mathbb{N}, d>1$, the range of $r$ was extended by Li and Xiao to $r>\frac{1}{2}$. Furthermore, they believe that with some special conditions on $\gamma$, the full range boundedness of $H_{\gamma}(f, g)$ and $M_{\gamma}(f, g)$ can be obtained. Here and hereafter, we omit the relationship that $p, q$, and $r$ satisfy $\frac{1}{p}+\frac{1}{q}=\frac{1}{r}, p>1$, and $q>1$ and the fact that $d>1$ and $d \in \mathbb{N}$. We call the full range bounded if the range of $r$ is $\left(\frac{1}{2}, \infty\right)$. In this paper, we provide some sufficient conditions of $\gamma$ regarding this concern.

Theorem 1.1. Let $\gamma \in C^{3}(\mathbb{R})$ be either odd or even, with $\gamma(0)=\gamma^{\prime}(0)=0, \lim _{t \rightarrow 0^{+}} \gamma^{\prime}(t) / \gamma^{\prime \prime}(t)=0$, and convex on $(0, \infty)$. Furthermore, if

$$
\text { there exist positive constants } C_{1} \text { and } C_{2} \text { such that } C_{1} \leq\left(\frac{\gamma^{\prime}}{\gamma^{\prime \prime}}\right)^{\prime}(t) \leq C_{2} \text { on }(0, \infty) \text {. }
$$

Then, there exists a positive constant $C$ such that

$$
\left\|H_{\gamma}(f, g)\right\|_{L^{r}(\mathbb{R})} \leq C\|f\|_{L^{p}(\mathbb{R})}\|g\|_{L^{q}(\mathbb{R})}
$$


for any $f \in L^{p}(\mathbb{R})$ and $g \in L^{q}(\mathbb{R})$, where $p, q$, r satisfy $\frac{1}{p}+\frac{1}{q}=\frac{1}{r}$, and $r>\frac{1}{2}, p>1, q>1$.

Theorem 1.2. Under the same conditions of $\gamma$, we have

$$
\left\|M_{\gamma}(f, g)\right\|_{L^{r}(\mathbb{R})} \leq C\|f\|_{L^{p}(\mathbb{R})}\|g\|_{L^{q}(\mathbb{R})} .
$$

Remark 1.3. It is easy to see that $H_{\gamma}(f, g)$ does not map $L^{\infty}(\mathbb{R}) \times L^{\infty}(\mathbb{R})$ into $L^{\infty}(\mathbb{R})$. Moreover, it is trivial that $M_{\gamma}(f, g)$ is bounded from $L^{\infty}(\mathbb{R}) \times L^{\infty}(\mathbb{R})$ into $L^{\infty}(\mathbb{R})$. Therefore, we can restrict the range of $r$ as $\left(\frac{1}{2}, \infty\right)$ in the rest of the paper.

Remark 1.4. The following are some curves satisfying the conditions of Theorem 1.1, we here write only the part for $t \in[0, \infty)$ based on its odd or even property:

(i) for any $t \in[0, \infty), \gamma_{1}(t):=t^{\alpha}$ under $\alpha \in(1, \infty)$;

(ii) for any $t \in[0, \infty), \gamma_{2}(t):=t^{\alpha} \log (1+t)$ under $\alpha \in(1, \infty)$;

(iii) for any $t \in[0, \infty)$ and $K \in \mathbb{N}, \gamma_{3}(t):=\sum_{i=1}^{K} t^{\alpha_{i}}$ under $\alpha_{i} \in(1, \infty)$ for all $i=1,2, \cdots, K$.

Remark 1.5. For a more general curve $\gamma$, Lie [39] introduced a set $\mathcal{N} \mathcal{F}^{C}$ and obtained the $L^{2}(\mathbb{R}) \times$ $L^{2}(\mathbb{R}) \rightarrow L^{1}(\mathbb{R})$ boundedness of $H_{\gamma}(f, g)$ for $\gamma \in \mathcal{N} \mathcal{F}^{C}$. Later, it was extended to the $L^{p}(\mathbb{R}) \times$ $L^{q}(\mathbb{R}) \rightarrow L^{r}(\mathbb{R})$ boundedness with $r \geq 1$ in [40]. Furthermore, Gaitan and Lie [20] obtained the same boundedness for $M_{\gamma}(f, g)$. It is worth noting that these results are sharp in the sense that we cannot take $\frac{1}{2}<r<1$, since the polynomial P stated in [37] belongs to the set $\mathcal{N F}^{C}$. More recently, Guo and Xiao [24] obtained the $L^{2}(\mathbb{R}) \times L^{2}(\mathbb{R}) \rightarrow L^{1}(\mathbb{R})$ boundedness of $H_{\gamma}(f, g)$ and $M_{\gamma}(f, g)$, where $\gamma \in \mathbf{F}(-1,1)$; the definition of the set $\mathbf{F}(-1,1)$ can be found on P. 970 in [24].

The argument of this paper is based on the works of Guo and Xiao [24], Li [34], Li and Xiao [37] and Lie [39, 40], but we also make several contributions:

$\star$ Our conditions may be easier to check than $\mathcal{N} \mathcal{F}^{C}$ in Lie [39, 40] and $\mathbf{F}(-1,1)$ in Guo and Xiao [24]. Moreover, we require less regularity on $\gamma$ than that in [39, 40, 24]. On the other hand, we only require that the curve $\gamma$ belongs to $C^{3}$, but $\mathcal{N \mathcal { F } ^ { C }} \subset C^{4}$ and $\mathbf{F}(-1,1) \subset C^{5}$.

$\star$ We obtain the full range boundedness for $H_{\gamma}(f, g)$ and $M_{\gamma}(f, g)$. Therefore, our results extend the results of Li [34] and Li and Xiao [37, Theorem 3], which concern the homogeneous curve $\gamma(t):=t^{d}$, to more general classes of curves.

$\star$ The main difference between this paper and the abovementioned works is a partition of unity. We split our multiplier by the following partition of unity; i.e.,

$$
\sum_{m, n, k \in \mathbb{Z}} \phi\left(\frac{\xi}{2^{m+j}}\right) \phi\left(\frac{\eta}{2^{k}}\right) \phi\left(\frac{\gamma^{\prime}\left(2^{-j}\right)}{2^{n+j-k}}\right)=1,
$$

see (2.25), instead of

$$
\sum_{m, n \in \mathbb{Z}} \phi\left(\frac{\xi}{2^{m+j}}\right) \phi\left(\frac{\eta \gamma^{\prime}\left(2^{-j}\right)}{2^{n+j}}\right)=1
$$


where $\phi$ is a standard bump function supported on $\left\{t \in \mathbb{R}: \frac{1}{2} \leq|t| \leq 2\right\}$ such that $0 \leq \phi(t) \leq 1$ and $\Sigma_{l \in \mathbb{Z}} \phi\left(2^{-l} t\right)=1$ for all $t \neq 0$. The aim of this partition of unity (2.25) is to avoid using uniform paraproduct estimates at the low-frequency part. In [34], Li used a uniform paraproduct estimate, i.e., [34, Theorem 4.1], to bound the low-frequency part. In this paper, we present an easy way to dispose of the low-frequency part by using this partition of unity and the Littlewood-Paley theory together with the uniform estimates (3.4) and (3.5).

\subsection{Background and motivation}

There are rich backgrounds from which to study the boundedness property of $H_{\gamma}(f, g)$ and $M_{\gamma}(f, g)$.

$\diamond$ If we take $\gamma(t):=t$, the boundedness of these two operators is trivial. This follows from the boundedness of the classical Hilbert transform, the Hardy-Littlewood maximal function and the Hölder inequality.

$\diamond$ If $\gamma(t):=-t$, these operators turn out to be the standard bilinear Hilbert transform and the corresponding (sub)bilinear maximal function whose boundedness is not easy to obtain. Lacey and Thiele [30, 31] obtained the boundedness with $r>\frac{2}{3}$ for the standard bilinear Hilbert transform. For the same boundedness of the corresponding maximal function, we refer to Lacey [29]. In the same paper, a counterexample showed that if $\frac{1}{2}<r<\frac{2}{3}$ the boundedness fails for these operators.

$\diamond$ If we take $\gamma(t):=t^{d}$ or $\gamma:=\mathrm{P}$ or a more general curve $\gamma$, the boundedness of these two operators has been stated at the beginning of this paper and in Remark 1.5, where $P$ is a polynomial of degree $d$ with no linear term and constant term.

$\diamond$ There are some other types of bilinear Hilbert transforms. Let

$$
H_{\alpha, \beta}(f, g)(x):=\mathrm{p} . \mathrm{v} \cdot \int_{-\infty}^{\infty} f(x-\alpha t) g(x-\beta t) \frac{\mathrm{d} t}{t} .
$$

Grafakos and $\mathrm{Li}$ [23] set up the uniform boundedness for $r>1$. Later, Li [35] extended the index to $r>\frac{2}{3}$. Recently, Dong [12] considered the bilinear Hilbert transform $H_{P, Q}(f, g)$ along two polynomials

$$
H_{\mathrm{P}, \mathrm{Q}}(f, g)(x):=\mathrm{p} \cdot \mathrm{v} \cdot \int_{-\infty}^{\infty} f(x-\mathrm{P}(t)) g(x-\mathrm{Q}(t)) \frac{\mathrm{d} t}{t}
$$

and the corresponding maximal operator $M_{P, Q}(f, g)$

$$
M_{\mathrm{P}, \mathrm{Q}}(f, g)(x):=\sup _{\varepsilon>0} \frac{1}{2 \varepsilon} \int_{-\varepsilon}^{\varepsilon}|f(x-\mathrm{P}(t)) g(x-\mathrm{Q}(t))| \mathrm{d} t .
$$

Here, $\mathrm{P}$ and $\mathrm{Q}$ are polynomials with no constant term. Dong proved that these operators are bounded for $r>\frac{d}{d+1}$, where $d$ is the correlation degree of these two polynomials P and Q. For the definition of the correlation degree, we refer the reader to P. 2 in [12]. There are many other related works; see, for example, [11, 13, 14, 15, 18, 32]. 
The study of the boundedness of $H_{\gamma}(f, g)$ and $M_{\gamma}(f, g)$ originated from Calderón [4] in order to study the Cauchy transform along Lipschitz curves, but there have also been many other motivations:

$\triangleright$ One of the motivations arises from ergodic theory. For instance, for $n \in \mathbb{N}$, the $L^{r}(\mathbb{R})$-norm convergence property of the non-conventional bilinear averages

$$
\frac{1}{N} \sum_{n=1}^{N} f\left(T^{n}\right) g\left(T^{n^{2}}\right)
$$

as $N$ tends to $\infty$. Here, $T$ is an invertible and measure-preserving transformation of a finite measure space. For more details, we refer to [10, 19, 27].

$\triangleright$ Another motivation is offered by number theory. There are many various nonlinear extensions of Roth's theorem for some sets with positive density; see, for example, [2, 3, 16].

$\triangleright$ There have also been many developments during the last few years regarding the Hilbert transform $H_{\gamma} f$ along the curve $\gamma$ defined as

$$
H_{\gamma} f\left(x_{1}, x_{2}\right):=\text { p.v. } \int_{-\infty}^{\infty} f\left(x_{1}-t, x_{2}-\gamma(t)\right) \frac{\mathrm{d} t}{t},
$$

and the corresponding maximal function $M_{\gamma} f$ along the curve $\gamma$ defined as

$$
M_{\gamma} f(x):=\sup _{\varepsilon>0} \frac{1}{2 \varepsilon} \int_{-\varepsilon}^{\varepsilon}\left|f\left(x_{1}-t, x_{2}-\gamma(t)\right)\right| \mathrm{d} t .
$$

These operators were initiated by Fabes and Rivière [17] and Jones [28] in order to understand the behavior of the constant-coefficient parabolic differential operators. Later, $H_{\gamma} f$ and $M_{\gamma} f$ were extended to cover more general classes of curves [5, 6, 7, 9, 41, 43]. $H_{\gamma}(f, g)$ is closely associated with $H_{\gamma} f$ since they have the same multiplier. Indeed, we can rewrite $H_{\gamma}(f, g)(x)$ as

$$
\int_{-\infty}^{\infty} \int_{-\infty}^{\infty} \hat{f}(\xi) \hat{g}(\eta) e^{i \xi x} e^{i \eta x}\left(\text { p. v. } \int_{-\infty}^{\infty} e^{-i \xi t} e^{-i \eta \gamma(t)} \frac{\mathrm{d} t}{t}\right) \mathrm{d} \xi \mathrm{d} \eta
$$

and $H_{\gamma} f\left(x_{1}, x_{2}\right)$ can be rewritten as

$$
\int_{-\infty}^{\infty} \int_{-\infty}^{\infty} \hat{f}(\xi, \eta) e^{i \xi x_{1}} e^{i \eta x_{2}}\left(\text { p. v. } \int_{-\infty}^{\infty} e^{-i \xi t} e^{-i \eta \gamma(t)} \frac{\mathrm{d} t}{t}\right) \mathrm{d} \xi \mathrm{d} \eta
$$

Therefore, we can find many similarities between the approaches of $H_{\gamma}(f, g)$ and $H_{\gamma} f$.

\subsection{Organization and notations}

We now present the structure of the rest of this paper. 
- In Section2 we give some preliminaries for our proof. Subsection 2.1provides two inequalities about the curve $\gamma$, which will be used repeatedly in our proof. Subsection 2.2 is devoted to splitting $H_{\gamma}(f, g)$ into the following three parts: the low-frequency part $H_{\gamma}^{1}(f, g)$; the highfrequency part away from the diagonal part $H_{\gamma}^{2}(f, g)$ and the high-frequency part near the diagonal part $H_{\gamma}^{3}(f, g)$; see (2.32) and (2.33). At the same time, we split $\frac{1}{t}=\sum_{j \in \mathbb{Z}} 2^{j} \rho\left(2^{j} t\right)$ and set up a uniform boundedness for each $H_{\gamma, j}(f, g)$, where the corresponding kernel is $2^{j} \rho\left(2^{j} t\right)$. A similar decomposition of $M_{\gamma}(f, g)$ can be found in Subsection 2.3, the lowfrequency part $M_{\gamma}^{1}(f, g)$, the high-frequency part far from the diagonal part $M_{\gamma}^{2}(f, g)$ and the high-frequency part close to the diagonal part $M_{\gamma}^{3}(f, g)$; see (2.42).

- In Section 3, we obtain the full range boundedness for $H_{\gamma}^{1}(f, g)$ by using Taylor series expansion.

- In Section 4, we establish the full range boundedness for $H_{\gamma}^{2}(f, g)$. To this aim, we consider two cases according to the function that has the higher frequency.

- In Section 5 , we prove the $L^{2}(\mathbb{R}) \times L^{2}(\mathbb{R}) \rightarrow L^{1}(\mathbb{R})$ boundedness of $H_{\gamma}^{3}(f, g)$. To this aim, we obtain a $2^{-\varepsilon_{0} m}$ decay for $H_{m}(f, g)$ (see $[6.2)$ ) defined on the frequency piece along the diagonal for some positive constants $\varepsilon_{0}$. Here, we used the $T T^{*}$ argument, Hörmander's theorem [26, Theorem 1.1], the stationary phase method and $\sigma$-uniformity.

- In Section 6, we obtain the full range weak- $L^{p}(\mathbb{R}) \times L^{q}(\mathbb{R}) \rightarrow L^{r}(\mathbb{R})$ boundedness for $H_{m}(f, g)$ with a bound $m$. By interpolation with the $L^{2}(\mathbb{R}) \times L^{2}(\mathbb{R}) \rightarrow L^{1}(\mathbb{R})$ estimate that has a decay bound of $2^{-\varepsilon_{0} m}$, we can finish our proof. To obtain the weak boundedness, we need to split $H_{m}(f, g)$ into the following three error terms: $\left|H_{m}^{1}\right|(f, g),\left|H_{m}^{2}\right|(f, g)$ and $\left|H_{m}^{3}\right|(f, g)$, see (6.8), and the major term $\left|H_{m}^{4}\right|(f, g)$ in 6.9). Subsection 6.1 describes the estimation for these three error terms, and Subsection 6.2 is devoted to establishing the major term $\left|H_{m}^{4}\right|(f, g)$ by using the method of time frequency analysis, which is the most difficult part.

- In Section 7, we set up the full range boundedness of $M_{\gamma}(f, g)$.

Throughout this paper, we denote by $C$ a positive constant that is independent of the main parameters involved, whose exact value is allowed to change from line to line. The positive constants with subscripts, $C_{1}$ and $C_{2}$, are fixed constants. The symbol $a \lesssim b$ or $b \gtrsim a$ means that there exists a positive constant $C$ such that $a \leq C b . a \approx b$ means $a \lesssim b$ and $b \lesssim a$. We use $\mathcal{S}(\mathbb{R})$ to denote the Schwartz class on $\mathbb{R}$. Let $\mathbb{Z}_{-}:=\mathbb{Z} \backslash \mathbb{N}$ with $\mathbb{N}:=\{0,1,2, \cdots\}$. $\hat{f}$ denotes the Fourier transform of $f$, and $\breve{f}$ is the inverse Fourier transform of $f$. For any $0<p<\infty$, we denote $p^{\prime}$ as its conjugate index if $\frac{1}{p}+\frac{1}{p^{\prime}}=1$. It is obvious that $p^{\prime}<0$ if $0<p<1$. For any set $E$, we use $\chi_{E}$ to denote its characteristic function. $\sharp E$ denotes the cardinality of it. $E^{\complement}$ indicates its complementary set.

Acknowledgments. The second author would like to thank his postdoctoral advisor Prof. Lixin Yan for the many valuable comments and helpful discussions. 


\section{Preliminaries}

\subsection{The curve $\gamma$}

We first explain some simple properties of the curve $\gamma$ in Theorem 1.1 which will be used in this paper. Since $\gamma \in C^{3}(\mathbb{R}), \gamma(0)=\gamma^{\prime}(0)=0$ and $\gamma$ is convex on $(0, \infty)$, we have $\gamma^{\prime \prime}(t) \geq 0$ on $(0, \infty)$. By (1.1), we obtain $\gamma^{\prime \prime}(t) \neq 0$ on $(0, \infty)$ and thus $\gamma^{\prime \prime}(t)>0$ on $(0, \infty)$. Therefore, $\gamma^{\prime}$ is strictly increasing and $\gamma^{\prime}(t)>0$ on $(0, \infty)$. On the other hand, let us set $G_{1}(t):=2 C_{2} t-\gamma^{\prime}(t) / \gamma^{\prime \prime}(t)$ and $G_{2}(t):=\gamma^{\prime}(t) / \gamma^{\prime \prime}(t)-C_{1} t / 2$, by 1.1], we then have $G_{1}^{\prime}(t) \geq C_{2}$ and $G_{2}^{\prime}(t) \geq C_{1} / 2$ on $(0, \infty)$. This, combined with $\lim _{t \rightarrow 0^{+}} \gamma^{\prime}(t) / \gamma^{\prime \prime}(t)=0$, leads to $G_{1}(t) \geq 0$ and $G_{2}(t) \geq 0$ on $(0, \infty)$ and therefore

$$
\frac{1}{2 C_{2}} \leq \frac{t \gamma^{\prime \prime}(t)}{\gamma^{\prime}(t)} \leq \frac{2}{C_{1}}, \quad \text { for any } t \in(0, \infty)
$$

Since $\gamma(0)=\gamma^{\prime}(0)=0$, by the Cauchy mean value theorem, for any $t \in(0, \infty)$, there exists $\tau_{1} \in(0, t)$ such that $\frac{t \gamma^{\prime}(t)}{\gamma(t)}=\frac{t \gamma^{\prime}(t)-0 \gamma^{\prime}(0)}{\gamma(t)-\gamma(0)}=\frac{\gamma^{\prime}\left(\tau_{1}\right)+\tau_{2} \gamma^{\prime \prime}\left(\tau_{1}\right)}{\gamma^{\prime}\left(\tau_{2}\right)}$. Thus, by 2.1),

$$
1+\frac{1}{2 C_{2}} \leq \frac{t \gamma^{\prime}(t)}{\gamma(t)} \leq 1+\frac{2}{C_{1}}, \quad \text { for any } t \in(0, \infty),
$$

which further implies that $\gamma(t) / t$ is strictly increasing on $(0, \infty)$.

Let $G_{3}(t):=\log _{2} \gamma^{\prime}(t)$ for any $t \in(0, \infty)$, by (2.1), we then have $1 / 2 C_{2} t \leq G_{3}^{\prime}(t) \leq 2 / C_{1} t$ for any $t \in(0, \infty)$. By the Lagrange mean value theorem, there exists a constant $\theta \in[1,2]$ such that $G_{3}(2 t)-G_{3}(t)=G_{3}^{\prime}(\theta t) t \in\left[1 / 4 C_{2}, 2 / C_{1}\right]$, which further leads to

$$
2^{\frac{1}{4 C_{2}}} \leq \frac{\gamma^{\prime}(2 t)}{\gamma^{\prime}(t)} \leq 2^{\frac{2}{C_{1}}}, \quad \text { for any } t \in(0, \infty)
$$

By the Cauchy mean value theorem and $\gamma(0)=0$, for any $t \in(0, \infty)$, there exists $\tau_{2} \in(0, t)$ such that $\frac{\gamma(2 t)}{\gamma(t)}=\frac{\gamma(2 t)-\gamma(0)}{\gamma(t)-\gamma(0)}=\frac{2 \gamma^{\prime}\left(2 \tau_{2}\right)}{\gamma^{\prime}\left(\tau_{2}\right)}$. This, combined with (2.3), implies

$$
2^{1+\frac{1}{4 C_{2}}} \leq \frac{\gamma(2 t)}{\gamma(t)} \leq 2^{1+\frac{2}{C_{1}}}, \quad \text { for any } t \in(0, \infty) .
$$

\subsection{Decomposition of $H_{\gamma}(f, g)$}

For $H_{\gamma}(f, g)$, we rewrite it as

$$
H_{\gamma}(f, g)(x):=\int_{-\infty}^{\infty} \int_{-\infty}^{\infty} \hat{f}(\xi) \hat{g}(\eta) e^{i \xi x} e^{i \eta x} m(\xi, \eta) \mathrm{d} \xi \mathrm{d} \eta
$$

where

$$
m(\xi, \eta):=\text { p. v. } \int_{-\infty}^{\infty} e^{-i \xi t} e^{-i \eta \gamma(t)} \frac{\mathrm{d} t}{t} .
$$

Let $\rho$ be an odd smooth function supported on $\left\{t \in \mathbb{R}: \frac{1}{2} \leq|t| \leq 2\right\}$ such that $\frac{1}{t}=\sum_{j \in \mathbb{Z}} 2^{j} \rho\left(2^{j} t\right)$. Then,

$$
m(\xi, \eta)=\sum_{j \in \mathbb{Z}} m_{j}(\xi, \eta)
$$


where

$$
m_{j}(\xi, \eta):=\int_{-\infty}^{\infty} e^{-i 2^{-j} \xi t} e^{-i \eta \gamma\left(2^{-j} t\right)} \rho(t) \mathrm{d} t
$$

Therefore, we split $H_{\gamma}(f, g)$ as

$$
H_{\gamma}(f, g)(x)=\sum_{j \in \mathbb{Z}} \int_{-\infty}^{\infty} \int_{-\infty}^{\infty} \hat{f}(\xi) \hat{g}(\eta) e^{i \xi x} e^{i \eta x} m_{j}(\xi, \eta) \mathrm{d} \xi \mathrm{d} \eta=: \sum_{j \in \mathbb{Z}} H_{\gamma, j}(f, g)(x) .
$$

From Proposition 2.2 below, we need only to consider that $|j|$ large enough. Before giving Proposition 2.2, we first state the following lemma which can be found in [25, Lemma 4.7].

Lemma 2.1. Let $I \subset \mathbb{R}$ be an interval, $k \in \mathbb{N}, f \in C^{k}(I)$, and suppose that for some $\sigma>0$, $\left|f^{(k)}(x)\right| \geq \sigma$ for all $x \in I$. Then there exists a positive constant $C$ depending only on $k$ such that

$$
|\{x \in I:|f(x)| \leq \rho\}| \leq C\left(\frac{\rho}{\sigma}\right)^{\frac{1}{k}}
$$

for all $\rho>0$.

Proposition 2.2. Let $\gamma$ and $p, q, r$ be the same as in Theorem 1.1 Then there exists a positive constant $C$ independent of $j$ such that

$$
\left\|H_{\gamma, j}(f, g)\right\|_{L^{r}(\mathbb{R})} \leq C\|f\|_{L^{p}(\mathbb{R})}\|g\|_{L^{q}(\mathbb{R})}
$$

for all $f \in L^{p}(\mathbb{R})$ and $g \in L^{q}(\mathbb{R})$.

Proof. For $r \geq 1$, by the Minkowski inequality and Hölder inequality, we have

$$
\left\|H_{\gamma, j}(f, g)\right\|_{L^{r}(\mathbb{R})} \leq \int_{-\infty}^{\infty}\left\||f(\cdot-t)|^{r}\right\|_{L^{\frac{p}{r}(\mathbb{R})}}^{\frac{1}{r}}\left\|\left.g(\cdot-\gamma(t))\right|^{r}\right\|_{L^{\frac{q}{r}(\mathbb{R})}}^{\frac{1}{r}} 2^{j}\left|\rho\left(2^{j} t\right)\right| \mathrm{d} t \lesssim\|f\|_{L^{p}(\mathbb{R})}\|g\|_{L^{q}(\mathbb{R})} .
$$

For $\frac{1}{2}<r<1$, we rewrite $H_{\gamma, j}(f, g)$ as

$$
H_{\gamma, j}(f, g)(x)=\int_{-\infty}^{\infty} f\left(x-2^{-j} t\right) g\left(x-\gamma\left(2^{-j} t\right)\right) \rho(t) \mathrm{d} t .
$$

Let

$$
\left\{\begin{array}{l}
A_{1}:=\left\{2^{-j} t: t \in \operatorname{supp} \rho\right\} \\
A_{\gamma}:=\left\{\gamma\left(2^{-j} t\right): t \in \operatorname{supp} \rho\right\} \\
E_{\lambda}:=\left\{t \in \operatorname{supp} \rho: 2^{\lambda}<2^{-j}\left|\gamma^{\prime}\left(2^{-j} t\right)\right| \leq 2 \cdot 2^{\lambda}\right\}
\end{array}\right.
$$

where $\lambda \in \mathbb{Z}$. Furthermore, let $A_{1}(\lambda):=\left\{2^{-j} t: t \in E_{\lambda}\right\}$ and $A_{\gamma}(\lambda):=\left\{\gamma\left(2^{-j} t\right): t \in E_{\lambda}\right\}$; we have

$$
H_{\gamma, j}(f, g)(x)=\sum_{\lambda \in \mathbb{Z}} \int_{E_{\lambda}} f\left(x-2^{-j} t\right) g\left(x-\gamma\left(2^{-j} t\right)\right) \rho(t) \mathrm{d} t=: \sum_{\lambda \in \mathbb{Z}} H_{\gamma, j}^{\lambda}(f, g)(x) .
$$

To estimate $H_{\gamma, j}(f, g)$, we consider the following three cases:

$$
2^{\lambda} \geq 2 \cdot 2^{-j}, \quad 2^{\lambda} \leq \frac{1}{4} 2^{-j} \quad \text { and } \quad \frac{1}{2} 2^{-j} \leq 2^{\lambda} \leq 2^{-j} .
$$


Case I: $2^{\lambda} \geq 2 \cdot 2^{-j}$

We observe that

$$
\left|A_{1}(\lambda)\right|=2^{-j}\left|E_{\lambda}\right| \leq 2^{\lambda}\left|E_{\lambda}\right| \quad \text { and } \quad\left|A_{\gamma}(\lambda)\right| \leq 2 \cdot 2^{\lambda}\left|E_{\lambda}\right| \text {. }
$$

Without loss of generality, we may restrict $x \in I_{\lambda}$ of length $2 \cdot 2^{\lambda}\left|E_{\lambda}\right|$. By the Cauchy-Schwarz inequality, we have

$$
\left\|H_{\gamma, j}^{\lambda}(f, g)\right\|_{L^{\frac{1}{2}(\mathbb{R})}}^{\frac{1}{2}} \leq\left|I_{\lambda}\right|^{\frac{1}{2}}\left(\int_{I_{\lambda}} \int_{E_{\lambda}}\left|f\left(x-2^{-j} t\right) g\left(x-\gamma\left(2^{-j} t\right)\right)\right| \mathrm{d} t \mathrm{~d} x\right)^{\frac{1}{2}} .
$$

We change the variables $u:=x-2^{-j} t$ and $v:=x-\gamma\left(2^{-j} t\right)$, and

$$
\left|\frac{\partial(u, v)}{\partial(x, t)}\right|=\left|2^{-j}-2^{-j} \gamma^{\prime}\left(2^{-j} t\right)\right| \geq 2^{\lambda}-\frac{2^{\lambda}}{2}=\frac{2^{\lambda}}{2}
$$

for all $t \in E_{\lambda}$. Note that $\left|I_{\lambda}\right|=2 \cdot 2^{\lambda}\left|E_{\lambda}\right|$; we can control the last term in 2.10) by

$$
\left(\frac{\left|I_{\lambda}\right|}{2^{\lambda}}\right)^{\frac{1}{2}}\left(\|f\|_{L^{1}(\mathbb{R})}\|g\|_{L^{1}(\mathbb{R})}\right)^{\frac{1}{2}} \approx\left|E_{\lambda}\right|^{\frac{1}{2}}\left(\|f\|_{L^{1}(\mathbb{R})}\|g\|_{L^{1}(\mathbb{R})}\right)^{\frac{1}{2}} .
$$

Henceforth, in this case,

$$
\left\|H_{\gamma, j}^{\lambda}(f, g)\right\|_{L^{\frac{1}{2}(\mathbb{R})}} \lesssim\left|E_{\lambda}\right| \cdot\|f\|_{L^{1}(\mathbb{R})}\|g\|_{L^{1}(\mathbb{R})} .
$$

For $E_{\lambda}$, by (2.1), we have

$$
2^{-2 j}\left|\gamma^{\prime \prime}\left(2^{-j} t\right)\right|=2^{-j}\left|\frac{2^{-j} t \gamma^{\prime \prime}\left(2^{-j} t\right)}{\gamma^{\prime}\left(2^{-j} t\right)}\right| \cdot\left|\frac{\gamma^{\prime}\left(2^{-j} t\right)}{t}\right| \gtrsim 2^{-j} \gamma^{\prime}\left(\frac{1}{2} 2^{-j}\right)
$$

for $t \in \operatorname{supp} \rho$. By Lemma 2.1, it implies

$$
\left|E_{\lambda}\right| \leq \frac{2^{\lambda}}{2^{-j} \gamma^{\prime}\left(\frac{1}{2} 2^{-j}\right)}
$$

On the other hand, since $\gamma^{\prime}$ is strictly increasing on $(0, \infty)$, we have

$$
2^{\lambda} \leq 2^{-j} \gamma^{\prime}\left(2 \cdot 2^{-j}\right)
$$

From (2.12), 2.13) and (2.3), we obtain

$$
\sum_{\lambda \in \mathbb{Z}: 2^{\lambda} \geq 2 \cdot 2^{-j}}\left|E_{\lambda}\right|^{\frac{1}{2}} \leq\left[\frac{1}{2^{-j} \gamma^{\prime}\left(\frac{1}{2} 2^{-j}\right)}\right]_{\lambda \in \mathbb{Z}: 2^{\lambda} \leq 2^{-j} \gamma^{\prime}\left(2 \cdot 2^{-j}\right)} 2^{\frac{\lambda}{2}} \lesssim\left[\frac{2^{-j} \gamma^{\prime}\left(2 \cdot 2^{-j}\right)}{2^{-j} \gamma^{\prime}\left(\frac{1}{2} 2^{-j}\right)}\right]^{\frac{1}{2}} \lesssim 1 .
$$

Therefore, from (2.11), 2.14), we have

$$
\left\|\sum_{\lambda \in \mathbb{Z}: 2^{\lambda} \geq 2 \cdot 2^{-j}} H_{\gamma, j}^{\lambda}(f, g)\right\|_{L^{\frac{1}{2}(\mathbb{R})}} \lesssim\|f\|_{L^{1}(\mathbb{R})}\|g\|_{L^{1}(\mathbb{R})} .
$$


As in (2.14), we have $\sum_{\lambda \in \mathbb{Z}: 2^{\lambda} \geq 2 \cdot 2^{-j}}\left|E_{\lambda}\right| \lesssim 1$, by the Hölder inequality, for all $p>1$,

$$
\left\|\sum_{\lambda \in \mathbb{Z}: 2^{\lambda} \geq 2 \cdot 2^{-j}} H_{\gamma, j}^{\lambda}(f, g)\right\|_{L^{1}(\mathbb{R})} \lesssim \sum_{\lambda \in \mathbb{Z}: 2^{\lambda} \geq 2 \cdot 2^{-j}}\left|E_{\lambda}\right| \cdot\|f\|_{L^{p}(\mathbb{R})}\|g\|_{L^{p^{\prime}}(\mathbb{R})} \lesssim\|f\|_{L^{p}(\mathbb{R})}\|g\|_{L^{p^{\prime}}(\mathbb{R})} .
$$

By interpolation between (2.15) and (2.16), for any $\frac{1}{2}<r<1$, we obtain

$$
\left\|\sum_{\lambda \in \mathbb{Z}: 2^{\lambda} \geq 2 \cdot 2^{-j}} H_{\gamma, j}^{\lambda}(f, g)\right\|_{L^{r}(\mathbb{R})} \lesssim\|f\|_{L^{p}(\mathbb{R})}\|g\|_{L^{q}(\mathbb{R})} .
$$

Case II: $2^{\lambda} \leq \frac{1}{4} 2^{-j}$

Noting that

$$
\left|A_{\gamma}(\lambda)\right| \leq 2 \cdot 2^{\lambda}\left|E_{\lambda}\right| \leq \frac{1}{2} 2^{-j}\left|E_{\lambda}\right|=\frac{1}{2}\left|A_{1}(\lambda)\right| \leq 2^{-j}\left|E_{\lambda}\right|,
$$

we restrict $x$ in an interval $I_{\lambda}$ of length $2 \cdot 2^{-j}\left|E_{\lambda}\right|$. On the other hand, we have

$$
\left|\frac{\partial(u, v)}{\partial(x, t)}\right|=\left|2^{-j}-2^{-j} \gamma^{\prime}\left(2^{-j} t\right)\right| \geq 2^{-j}-2 \cdot 2^{\lambda} \geq \frac{1}{2} 2^{-j}
$$

for all $t \in E_{\lambda}$. As in Case I, we also have

$$
\left\|H_{\gamma, j}^{\lambda}(f, g)\right\|_{L^{\frac{1}{2}(\mathbb{R})}} \lesssim\left|E_{\lambda}\right| \cdot\|f\|_{L^{1}(\mathbb{R})}\|g\|_{L^{1}(\mathbb{R})} .
$$

Furthermore,

$$
\left\|\sum_{\lambda \in \mathbb{Z}: 2^{\lambda} \leq \frac{1}{4} 2^{-j}} H_{\gamma, j}^{\lambda}(f, g)\right\|_{L^{r}(\mathbb{R})} \lesssim\|f\|_{L^{p}(\mathbb{R})}\|g\|_{L^{q}(\mathbb{R})}
$$

for all $\frac{1}{2}<r<1$.

Case III: $\frac{1}{2} 2^{-j} \leq 2^{\lambda} \leq 2^{-j}$

We are free to assume that $2^{-j} \gamma^{\prime}\left(2^{-j} t\right)>0$. Otherwise, it can be handled exactly in the same way as Case II, since

$$
\left|A_{\gamma}(\lambda)\right| \leq 2 \cdot 2^{-j}\left|E_{\lambda}\right|, \quad\left|A_{1}(\lambda)\right|=2^{-j}\left|E_{\lambda}\right|
$$

and

$$
\left|\frac{\partial(u, v)}{\partial(x, t)}\right|=\left|2^{-j}-2^{-j} \gamma^{\prime}\left(2^{-j} t\right)\right| \geq 2^{-j}
$$

for $t \in E_{\lambda}$. We now consider

$$
\mathbb{H}_{\gamma, j}(f, g)(x):=\int_{E_{\gamma}} f\left(x-2^{-j} t\right) g\left(x-\gamma\left(2^{-j} t\right)\right) \rho(t) \mathrm{d} t
$$

where $E_{\gamma}:=\left\{t \in \operatorname{supp} \rho: \frac{1}{2}<\gamma^{\prime}\left(2^{-j} t\right) \leq 2\right\}$. Let $E_{\gamma}(h):=\left\{t \in E_{\gamma}: 2^{h}<\left|\gamma^{\prime}\left(2^{-j} t\right)-1\right| \leq 2 \cdot 2^{h}\right\}$. By simple calculation, we know that $E_{\gamma} \subset \bigcup_{h \in \mathbb{Z}_{-}} E_{\gamma}(h)$. Let

$$
\mathbb{H}_{\gamma, j}^{h}(f, g)(x):=\int_{E_{\gamma}(h)} f\left(x-2^{-j} t\right) g\left(x-\gamma\left(2^{-j} t\right)\right) \rho(t) \mathrm{d} t .
$$


Noting that $\left|\left\{2^{-j} t: t \in E_{\gamma}(h)\right\}\right|=2^{-j}\left|E_{\gamma}(h)\right|$ and $\left|\left\{\gamma\left(2^{-j} t\right): t \in E_{\gamma}(h)\right\}\right| \lesssim 2^{-j}\left|E_{\gamma}(h)\right|$, without loss of generality, we restrict $x \in I_{h}$ of length $2^{-j}\left|E_{\gamma}(h)\right|$. By the Hölder inequality, for all $p>1$, we can bound $\left\|\mathbb{H}_{\gamma, j}^{h}(f, g)\right\|_{L^{1}(\mathbb{R})}$ by

$$
\int_{I_{h}}\left|\int_{E_{\gamma}(h)} f\left(x-2^{-j} t\right) g\left(x-\gamma\left(2^{-j} t\right)\right) \rho(t) \mathrm{d} t\right| \mathrm{d} x \leq\left|E_{\gamma}(h)\right| \cdot\|f\|_{L^{p}(\mathbb{R})}\|g\|_{L^{p^{\prime}}(\mathbb{R})} .
$$

The Cauchy-Schwarz inequality allows us to obtain

$$
\left\|\mathbb{H}_{\gamma, j}^{h}(f, g)\right\|_{L^{\frac{1}{2}(\mathbb{R})}}^{\frac{1}{2}} \lesssim\left|I_{h}\right|^{\frac{1}{2}}\left[\int_{I_{h}} \int_{E_{\gamma}(h)}\left|f\left(x-2^{-j} t\right) g\left(x-\gamma\left(2^{-j} t\right)\right)\right| \mathrm{d} t \mathrm{~d} x\right]^{\frac{1}{2}} .
$$

Let $u:=x-2^{-j} t$ and $v:=x-\gamma\left(2^{-j} t\right)$, noting that $\left|\frac{\partial(u, v)}{\partial(x, t)}\right|=\left|2^{-j}-2^{-j} \gamma^{\prime}\left(2^{-j} t\right)\right| \geq 2^{h} 2^{-j}$ for all $t \in E_{\gamma}(h)$ and $\left|I_{h}\right|=2^{-j}\left|E_{\gamma}(h)\right|$, we control the last term in $(2.21)$ by $\left(\frac{\left|E_{\gamma}(h)\right|}{2^{h}}\right)^{\frac{1}{2}}\left(\|f\|_{L^{1}(\mathbb{R})}\|g\|_{L^{1}(\mathbb{R})}\right)^{\frac{1}{2}}$. Henceforth, in this case,

$$
\left\|\mathbb{H}_{\gamma, j}^{h}(f, g)\right\|_{L^{\frac{1}{2}(\mathbb{R})}} \lesssim \frac{\left|E_{\gamma}(h)\right|}{2^{h}}\|f\|_{L^{1}(\mathbb{R})}\|g\|_{L^{1}(\mathbb{R})} .
$$

Noting that $\left|E_{\gamma}(h)\right| \leq 4$, from (2.20) and (2.22), by interpolation, we obtain the boundedness of $\mathbb{H}_{\gamma, j}^{h}(f, g)$ for all $\frac{1}{2}<r<1$ for the case that $h=-1$. Noting that $E_{\gamma} \subset \bigcup_{h \in \mathbb{Z}_{-}} E_{\gamma}(h)$, in what follows, we will focus on the second case; i.e., $h \in \mathbb{Z}_{-}$and $h<-1$.

From the fact that $\gamma^{\prime}$ is strictly increasing on $(0, \infty)$ and $(2.1)$, we have $2^{-j}\left|\gamma^{\prime \prime}\left(2^{-j} t\right)\right| \gtrsim \gamma^{\prime}\left(\frac{1}{2} 2^{-j}\right)$ for $t \in E_{\gamma}(h)$. By Lemma2.1, we have $\left|E_{\gamma}(h)\right| \lesssim \frac{2^{h}}{\gamma^{\prime}\left(\frac{1}{2} 2^{-j}\right)}$. Furthermore, we have

$$
\gamma^{\prime}\left(2 \cdot 2^{-j}\right) \geq\left|\gamma^{\prime}\left(2^{-j} t\right)\right|=\left|\gamma^{\prime}\left(2^{-j} t\right)-1+1\right| \geq 1-2 \cdot 2^{h} \geq \frac{1}{2}
$$

for $t \in E_{\gamma}(h)$. Therefore, (2.3) implies $\left|E_{\gamma}(h)\right| \lesssim \frac{2^{h}}{\gamma^{\prime}\left(2 \cdot 2^{-j}\right)} \frac{\gamma^{\prime}\left(2 \cdot 2^{-j}\right)}{\gamma^{\prime}\left(\frac{1}{2} 2^{-j}\right)} \lesssim 2^{h}$. By interpolation (2.20) and (2.22), there exists a positive constant $\epsilon$ independent of $j$ such that

$$
\left\|\mathbb{H}_{\gamma, j}^{h}(f, g)\right\|_{L^{r}(\mathbb{R})} \lesssim 2^{\epsilon h}\|f\|_{L^{p}(\mathbb{R})}\|g\|_{L^{q}(\mathbb{R})}
$$

for $\frac{1}{2}<r<1$, which leads to

$$
\left\|\sum_{h \in \mathbb{Z}_{-}: h<-1} \mathbb{H}_{\gamma, j}^{h}(f, g)\right\|_{L^{r}(\mathbb{R})} \lesssim\|f\|_{L^{p}(\mathbb{R})}\|g\|_{L^{q}(\mathbb{R})} .
$$

Putting all the estimates together, we finish the proof of Proposition 2.2

Let $\phi$ be a standard bump function supported on $\left\{t \in \mathbb{R}: \frac{1}{2} \leq|t| \leq 2\right\}$ such that $0 \leq \phi(t) \leq 1$ and $\Sigma_{l \in \mathbb{Z}} \phi\left(2^{-l} t\right)=1$ for all $t \neq 0$. We decompose the unity as

$$
\sum_{m, n, k \in \mathbb{Z}} \phi\left(\frac{\xi}{2^{m+j}}\right) \phi\left(\frac{\eta}{2^{k}}\right) \phi\left(\frac{\gamma^{\prime}\left(2^{-j}\right)}{2^{n+j-k}}\right)=1
$$


for all $\xi, \eta \neq 0$. Let

$$
m_{j, m, n, k}(\xi, \eta):=m_{j}(\xi, \eta) \phi\left(\frac{\xi}{2^{m+j}}\right) \phi\left(\frac{\eta}{2^{k}}\right) \phi\left(\frac{\gamma^{\prime}\left(2^{-j}\right)}{2^{n+j-k}}\right) .
$$

Then

$$
m_{j}(\xi, \eta)=\sum_{m, n, k \in \mathbb{Z}} m_{j, m, n, k}(\xi, \eta)
$$

We denote the diagonal as

$$
\Delta:=\left\{(m, n) \in \mathbb{Z}^{2}: m, n \geq 0,|m-n| \leq 2 / C_{1}+1\right\},
$$

and split $m_{j}$ as the following three parts:

$$
m_{j}(\xi, \eta)=m_{j}^{1}(\xi, \eta)+m_{j}^{2}(\xi, \eta)+m_{j}^{3}(\xi, \eta)
$$

Accordingly, the low-frequency part $m_{j}^{1}$ is

$$
m_{j}^{1}(\xi, \eta):=\sum_{(m, n) \in\left(\mathbb{Z}_{-}\right)^{2}, k \in \mathbb{Z}} m_{j, m, n, k}(\xi, \eta) .
$$

The high-frequency part away from the diagonal part $m_{j}^{2}$ is

$$
m_{j}^{2}(\xi, \eta):=\sum_{(m, n) \in \mathbb{Z}^{2} \backslash\left(\left(\mathbb{Z}_{-}\right)^{2} \cup \Delta\right), k \in \mathbb{Z}} m_{j, m, n, k}(\xi, \eta) .
$$

The high-frequency part near the diagonal part $m_{j}^{3}$ is

$$
m_{j}^{3}(\xi, \eta):=\sum_{(m, n) \in \Delta, k \in \mathbb{Z}} m_{j, m, n, k}(\xi, \eta) .
$$

Accordingly, we can split $H_{\gamma}(f, g)$ into three parts:

$$
H_{\gamma}(f, g)(x)=H_{\gamma}^{1}(f, g)(x)+H_{\gamma}^{2}(f, g)(x)+H_{\gamma}^{3}(f, g)(x),
$$

where

$$
\left\{\begin{array}{l}
H_{\gamma}^{1}(f, g)(x):=\sum_{j \in \mathbb{Z}} \int_{-\infty}^{\infty} \int_{-\infty}^{\infty} \hat{f}(\xi) \hat{g}(\eta) e^{i \xi x} e^{i \eta x} m_{j}^{1}(\xi, \eta) \mathrm{d} \xi \mathrm{d} \eta \\
H_{\gamma}^{2}(f, g)(x):=\sum_{j \in \mathbb{Z}} \int_{-\infty}^{\infty} \int_{-\infty}^{\infty} \hat{f}(\xi) \hat{g}(\eta) e^{i \xi x} e^{i \eta x} m_{j}^{2}(\xi, \eta) \mathrm{d} \xi \mathrm{d} \eta \\
H_{\gamma}^{3}(f, g)(x):=\sum_{j \in \mathbb{Z}} \int_{-\infty}^{\infty} \int_{-\infty}^{\infty} \hat{f}(\xi) \hat{g}(\eta) e^{i \xi x} e^{i \eta x} m_{j}^{3}(\xi, \eta) \mathrm{d} \xi \mathrm{d} \eta
\end{array}\right.
$$




\subsection{Decomposition of $M_{\gamma}(f, g)$}

$M_{\gamma}(f, g)$ is a positive operator, and we may assume that $f$ and $g$ are non-negative. By simple calculation, we deduce that

$$
M_{\gamma}(f, g)(x) \leq \sup _{j \in \mathbb{Z}} \int_{-\infty}^{\infty} f(x-t) g(x-\gamma(t)) 2^{j}\left|\rho\left(2^{j} t\right)\right| \mathrm{d} t=: \sup _{j \in \mathbb{Z}} M_{j, \gamma}(f, g)(x) .
$$

As in Proposition 2.2 above, we will focus on the case where $|j|$ is large enough. We rewrite $M_{j, \gamma}(f, g)$ as

$$
M_{j, \gamma}(f, g)(x):=\int_{-\infty}^{\infty} \int_{-\infty}^{\infty} \hat{f}(\xi) \hat{g}(\eta) e^{i \xi x} e^{i \eta x} \tilde{m}_{j}(\xi, \eta) \mathrm{d} \xi \mathrm{d} \eta
$$

where

$$
\tilde{m}_{j}(\xi, \eta):=\text { p.v. } \int_{-\infty}^{\infty} e^{-i 2^{-j} \xi t} e^{-i \eta \gamma\left(2^{-j} t\right)}|\rho(t)| \mathrm{d} t
$$

As in (2.26), we define $\tilde{m}_{j, m, n, k}$ as

$$
\tilde{m}_{j, m, n, k}(\xi, \eta):=\tilde{m}_{j}(\xi, \eta) \phi\left(\frac{\xi}{2^{m+j}}\right) \phi\left(\frac{\eta}{2^{k}}\right) \phi\left(\frac{\gamma^{\prime}\left(2^{-j}\right)}{2^{n+j-k}}\right),
$$

and split $\tilde{m}_{j}$ as

$$
\tilde{m}_{j}(\xi, \eta)=\tilde{m}_{j}^{1}(\xi, \eta)+\tilde{m}_{j}^{2}(\xi, \eta)+\tilde{m}_{j}^{3}(\xi, \eta)
$$

The low-frequency part $\tilde{m}_{j}^{1}$ is

$$
\tilde{m}_{j}^{1}(\xi, \eta):=\sum_{(m, n) \in\left(\mathbb{Z}_{-}\right)^{2}, k \in \mathbb{Z}} \tilde{m}_{j, m, n, k}(\xi, \eta) .
$$

The high-frequency part away from the diagonal part $\tilde{m}_{j}^{2}$ is

$$
\tilde{m}_{j}^{2}(\xi, \eta):=\sum_{(m, n) \in \mathbb{Z}^{2} \backslash\left(\left(\mathbb{Z}_{-}\right)^{2} \cup \Delta\right), k \in \mathbb{Z}} \tilde{m}_{j, m, n, k}(\xi, \eta) .
$$

The high-frequency part near the diagonal part $\tilde{m}_{j}^{3}$ is

$$
\tilde{m}_{j}^{3}(\xi, \eta):=\sum_{(m, n) \in \Delta, k \in \mathbb{Z}} \tilde{m}_{j, m, n, k}(\xi, \eta)
$$

Similarly,

$$
M_{\gamma}(f, g)(x) \leq M_{\gamma}^{1}(f, g)(x)+M_{\gamma}^{2}(f, g)(x)+M_{\gamma}^{3}(f, g)(x) .
$$




\section{The boundedness of $H_{\gamma}^{1}(f, g)$}

For $m_{j}^{1}$ in 2.29), we employ the Taylor series expansion, i.e., $e^{-i 2^{-j} \xi t}=\sum_{u \in \mathbb{N}} \frac{\left(-i 2^{-j} \xi t\right)^{u}}{u !}$ and $e^{-i \eta \gamma\left(2^{-j} t\right)}=\sum_{v \in \mathbb{N}} \frac{\left(-i \eta \gamma\left(2^{-j} t\right)\right)^{v}}{v !}$. Since $\phi$ is supported on $\left\{t \in \mathbb{R}: \frac{1}{2} \leq|t| \leq 2\right\}$, we have $\left|\frac{\xi}{2^{m+j}}\right| \leq 2$ and $\left|\frac{\eta \gamma^{\prime}\left(2^{-j}\right)}{2^{n+j}}\right|=\left|\frac{\eta}{2^{k}}\right| \cdot\left|\frac{\eta \gamma^{\prime}\left(2^{-j}\right)}{2^{n+j-k}}\right| \leq 4$. Noting that $\rho$ is supported on $\left\{t \in \mathbb{R}: \frac{1}{2} \leq|t| \leq 2\right\}$ and $m \in \mathbb{Z}_{-}$, we have $\left|2^{-j} \xi t\right| \leq 4 \cdot 2^{m} \leq 4$. On the other hand, since $\gamma$ is either odd or even and increasing on $(0, \infty)$ and satisfies (2.3) and (2.2), and $\rho$ is supported on $\left\{t \in \mathbb{R}: \frac{1}{2} \leq|t| \leq 2\right\}, n \in \mathbb{Z}_{-}$, we have

$$
\left|\eta \gamma\left(2^{-j} t\right)\right| \lesssim\left|\eta \gamma\left(2^{-j}\right)\right| \lesssim\left|\eta 2^{-j} \gamma^{\prime}\left(2^{-j}\right)\right| \lesssim 1 .
$$

Furthermore, let

$$
\bar{\phi}_{u}(\xi):=\xi^{u} \phi(\xi) \quad \text { and } \quad \psi_{\lambda}(\xi):=2^{\lambda} \psi\left(2^{\lambda} \xi\right),
$$

where $u, \lambda \in \mathbb{Z}$. Then, we can write $m_{j}^{1}(\xi, \eta)$ as

$$
\sum_{m, n \in \mathbb{Z}_{-}} \sum_{k \in \mathbb{Z}} \sum_{u, v \in \mathbb{N}} \frac{(-i)^{u+v} 2^{m u} 2^{n v}}{u ! v !} \bar{\phi}_{u}\left(\frac{\xi}{2^{m+j}}\right) \bar{\phi}_{v}\left(\frac{\eta}{2^{k}}\right) \bar{\phi}_{v}\left(\frac{\gamma^{\prime}\left(2^{-j}\right)}{2^{n+j-k}}\right) \int_{-\infty}^{\infty} t^{u}\left(\frac{2^{j} \gamma\left(2^{-j} t\right)}{\gamma^{\prime}\left(2^{-j}\right)}\right)^{v} \rho(t) \mathrm{d} t
$$

Therefore, $H_{\gamma}^{1}(f, g)(x)$ can be rewritten as

$$
\sum_{j, k \in \mathbb{Z}} \sum_{m, n \in \mathbb{Z}_{-}} \sum_{u, v \in \mathbb{N}} \frac{(-i)^{u+v} 2^{m u} 2^{n v}}{u ! v !} \bar{\phi}_{v}\left(\frac{\gamma^{\prime}\left(2^{-j}\right)}{2^{n+j-k}}\right) \int_{-\infty}^{\infty} t^{u}\left(\frac{2^{j} \gamma\left(2^{-j} t\right)}{\gamma^{\prime}\left(2^{-j}\right)}\right)^{v} \rho(t) \mathrm{d} t \cdot \check{\bar{\phi}}_{u, m+j} * f(x) \cdot \check{\bar{\phi}}_{v, k} * g(x),
$$

where $\check{\bar{\phi}}_{u, \lambda}(\xi):=2^{\lambda} \check{\bar{\phi}}_{u}\left(2^{\lambda} \xi\right)$ and $\check{\bar{\phi}}_{u}$ means the inverse Fourier transform of $\bar{\phi}_{u}$; by the CauchySchwarz inequality, we dominate $H_{\gamma}^{1}(f, g)(x)$ by

$$
\begin{gathered}
\sum_{m, n \in \mathbb{Z}} \sum_{u, v \in \mathbb{N}} \frac{2^{m u} 2^{n v}}{u ! v !}\left|\int_{-\infty}^{\infty} t^{u}\left(\frac{2^{j} \gamma\left(2^{-j} t\right)}{\gamma^{\prime}\left(2^{-j}\right)}\right)^{v} \rho(t) \mathrm{d} t\right| \\
\times\left[\sum_{j, k \in \mathbb{Z}}\left|\bar{\phi}_{v}\left(\frac{\gamma^{\prime}\left(2^{-j}\right)}{2^{n+j-k}}\right)\right| \cdot\left|\check{\bar{\phi}}_{u, m+j} * f(x)\right|^{2}\right]^{\frac{1}{2}} \cdot\left[\sum_{j, k \in \mathbb{Z}}\left|\bar{\phi}_{v}\left(\frac{\gamma^{\prime}\left(2^{-j}\right)}{2^{n+j-k}}\right)\right| \cdot\left|\check{\bar{\phi}}_{v, k} * g(x)\right|^{2}\right]^{\frac{1}{2}} .
\end{gathered}
$$

For $r \geq 1$, by the triangle inequality and Hölder inequality, the $L^{r}(\mathbb{R})$ norm of $H_{\gamma}^{1}(f, g)$ is at most

$$
\begin{aligned}
& \sum_{m, n \in \mathbb{Z}_{-}} \sum_{u, v \in \mathbb{N}} \frac{2^{m u} 2^{n v}}{u ! v !}\left|\int_{-\infty}^{\infty} t^{u}\left(\frac{2^{j} \gamma\left(2^{-j} t\right)}{\gamma^{\prime}\left(2^{-j}\right)}\right)^{v} \rho(t) \mathrm{d} t\right| \\
&\left.\times \|\left.\left|\sum_{j, k \in \mathbb{Z}}\right| \bar{\phi}_{v}\left(\frac{\gamma^{\prime}\left(2^{-j}\right)}{2^{n+j-k}}\right)|\cdot| \check{\bar{\phi}}_{u, m+j} * f\right|^{2}\right]^{\frac{1}{2}}\|\|_{L^{p}(\mathbb{R})} \|\left[\left.\sum_{j, k \in \mathbb{Z}}\left|\bar{\phi}_{v}\left(\frac{\gamma^{\prime}\left(2^{-j}\right)}{2^{n+j-k}}\right)\right| \cdot\left|\check{\bar{\phi}}_{v, k} * g\right|^{2}\right|^{\frac{1}{2} \|} \|_{L^{q}(\mathbb{R})} .\right.
\end{aligned}
$$

From (2.3) and (2.2), it is easy to see that

$$
\left|\int_{-\infty}^{\infty} t^{u}\left(\frac{2^{j} \gamma\left(2^{-j} t\right)}{\gamma^{\prime}\left(2^{-j}\right)}\right)^{v} \rho(t) \mathrm{d} t\right| \lesssim 1
$$


We claim that

$$
\sum_{j \in \mathbb{Z}}\left|\phi\left(\frac{\gamma^{\prime}\left(2^{-j}\right)}{2^{n+j-k}}\right)\right| \lesssim 1
$$

holds uniformly for $n \in \mathbb{Z}_{-}$and $k \in \mathbb{Z}$. Since $0 \leq \phi \leq 1$, it is enough to show that the sum in (3.4) has at most a finite number of terms and the number independent of $n \in \mathbb{Z}$ - and $k \in \mathbb{Z}$. Indeed, since $\phi$ is supported on $\left\{t \in \mathbb{R}: \frac{1}{2} \leq|t| \leq 2\right\}$, then, for the $j$-th term in (3.4), it implies $\frac{1}{2} \frac{2^{j}}{\gamma^{\prime}\left(2^{-j}\right)} \leq \frac{1}{2^{n-k}} \leq 2 \frac{2^{j}}{\gamma^{\prime}\left(2^{-j}\right)}$. For the $j+K$-th term in (3.4), it implies $\frac{1}{2} \frac{2^{j+K}}{\gamma^{\prime}\left(2^{-j-K}\right)} \leq \frac{1}{2^{n-k}} \leq 2 \frac{2^{j+K}}{\gamma^{\prime}\left(2^{-j-K}\right)}$, where $K>0$. Therefore, it suffices to show that there exists a positive constant $K$ that depends only on $\gamma$ such that $2 \frac{2^{j}}{\gamma^{\prime}\left(2^{-j}\right)}<\frac{1}{2} \frac{2^{j+K}}{\gamma^{\prime}\left(2^{-j-K}\right)}$. From (2.2), this is a direct consequence of $\frac{\gamma\left(2^{-j}\right)}{\gamma\left(2^{-j-K}\right)}>4 \frac{1+\frac{2}{C_{1}}}{1+\frac{1}{2 C_{2}}}$. From (2.4), we need only to take $K$ that satisfies $2^{\left(1+\frac{1}{4 C_{2}}\right) K}>4 \frac{1+\frac{2}{C_{1}}}{1+\frac{1}{2 C_{2}}}$.

As in (3.4), we also have that

$$
\sum_{k \in \mathbb{Z}}\left|\phi\left(\frac{\gamma^{\prime}\left(2^{-j}\right)}{2^{n+j-k}}\right)\right| \lesssim 1
$$

holds uniformly for $n \in \mathbb{Z}_{-}$and $j \in \mathbb{Z}$. (3.5) and the Littlewood-Paley theory implies

$$
\left\|\left[\sum_{j, k \in \mathbb{Z}}\left|\bar{\phi}_{v}\left(\frac{\gamma^{\prime}\left(2^{-j}\right)}{2^{n+j-k}}\right)\right| \cdot\left|\check{\bar{\phi}}_{u, m+j} * f\right|^{2}\right]^{\frac{1}{2}}\right\|_{L^{p}(\mathbb{R})} \lesssim\left\|\left[\sum_{j \in \mathbb{Z}}\left|\check{\bar{\phi}}_{u, m+j} * f\right|^{2}\right]^{\frac{1}{2}}\right\|_{L^{p}(\mathbb{R})} \lesssim\|f\|_{L^{p}(\mathbb{R})} .
$$

From (3.4), as in (3.6),

$$
\left\|\left[\sum_{j, k \in \mathbb{Z}}\left|\bar{\phi}_{v}\left(\frac{\gamma^{\prime}\left(2^{-j}\right)}{2^{n+j-k}}\right)\right| \cdot\left|\check{\bar{\phi}}_{v, k} * g\right|^{2}\right]^{\frac{1}{2}}\right\|_{L^{q}(\mathbb{R})} \lesssim\|g\|_{L^{q}(\mathbb{R})} .
$$

From the fact that $\sum_{m, n \in \mathbb{Z}_{-}} \sum_{u, v \in \mathbb{N}} \frac{2^{m u} 2^{n v}}{u ! v !} \lesssim 1$ and the estimates (3.2), (3.3), 3.6) and (3.7), we have

$$
\left\|H_{\gamma}^{1}(f, g)\right\|_{L^{r}(\mathbb{R})} \lesssim\|f\|_{L^{p}(\mathbb{R})}\|g\|_{L^{q}(\mathbb{R})} .
$$

For $\frac{1}{2}<r<1$, from 3.1 , we bound $\left\|H_{\gamma}^{1}(f, g)\right\|_{L^{r}(\mathbb{R})}^{r}$ by

$$
\begin{gathered}
\sum_{m, n \in \mathbb{Z}_{-}} \sum_{u, v \in \mathbb{N}}\left(\frac{2^{m u} 2^{n v}}{u ! v !}\right)^{r}\left|\int_{-\infty}^{\infty} t^{u}\left(\frac{2^{j} \gamma\left(2^{-j} t\right)}{\gamma^{\prime}\left(2^{-j}\right)}\right)^{v} \rho(t) \mathrm{d} t\right|^{r} \\
\times\left\|\left[\sum_{\sum_{j, k \in \mathbb{Z}}}\left|\bar{\phi}_{v}\left(\frac{\gamma^{\prime}\left(2^{-j}\right)}{2^{n+j-k}}\right)\right| \cdot\left|\check{\bar{\phi}}_{u, m+j} * f\right|^{2}\right]^{\frac{1}{2}} \cdot\left[\sum_{j, k \in \mathbb{Z}}\left|\bar{\phi}_{v}\left(\frac{\gamma^{\prime}\left(2^{-j}\right)}{2^{n+j-k}}\right)\right| \cdot\left|\check{\bar{\phi}}_{v, k} * g\right|^{2}\right]^{\frac{1}{2}}\right\|_{L^{r}(\mathbb{R})}^{r} .
\end{gathered}
$$

The fact $\sum_{m, n \in \mathbb{Z}_{-}} \sum_{u, v \in \mathbb{N}}\left(\frac{2^{m u} 2^{n v}}{u ! v !}\right)^{r} \lesssim 1$ for all $\frac{1}{2}<r<1$ combining (3.3), (3.6), (3.7) and (3.9), leads to

$$
\left\|H_{\gamma}^{1}(f, g)\right\|_{L^{r}(\mathbb{R})} \lesssim\|f\|_{L^{p}(\mathbb{R})}\|g\|_{L^{q}(\mathbb{R})} .
$$

This is the desired estimate for the first item $H_{\gamma}^{1}(f, g)$. 


\section{The boundedness of $H_{\gamma}^{2}(f, g)$}

Noting that

$$
\frac{i}{2^{-j} \xi+\eta 2^{-j} \gamma^{\prime}\left(2^{-j} t\right)} \frac{\mathrm{d}}{\mathrm{d} t} e^{-i 2^{-j} \xi t-i \eta \gamma\left(2^{-j} t\right)}=e^{-i 2^{-j} \xi t-i \eta \gamma\left(2^{-j} t\right)},
$$

we split $m_{j, m, n, k}$ in (2.26) as the following two parts:

$$
m_{j, m, n, k}(\xi, \eta)=A_{j, m, n, k}(\xi, \eta)+B_{j, m, n, k}(\xi, \eta),
$$

where $A_{j, m, n, k}$ is defined as

$$
\left(\int_{-\infty}^{\infty} e^{-i 2^{-j} \xi t-i \eta \gamma\left(2^{-j} t\right)} \frac{-i \rho^{\prime}(t)}{2^{-j} \xi+\eta 2^{-j} \gamma^{\prime}\left(2^{-j} t\right)} \mathrm{d} t\right) \phi\left(\frac{\xi}{2^{m+j}}\right) \phi\left(\frac{\eta}{2^{k}}\right) \phi\left(\frac{\gamma^{\prime}\left(2^{-j}\right)}{2^{n+j-k}}\right)
$$

and $B_{j, m, n, k}$ is defined as

$$
\left(\int_{-\infty}^{\infty} e^{-i 2^{-j} \xi t-i \eta \gamma\left(2^{-j} t\right)} \frac{i \eta 2^{-2 j} \gamma^{\prime \prime}\left(2^{-j} t\right)}{\left(2^{-j} \xi+\eta 2^{-j} \gamma^{\prime}\left(2^{-j} t\right)\right)^{2}} \rho(t) \mathrm{d} t\right) \phi\left(\frac{\xi}{2^{m+j}}\right) \phi\left(\frac{\eta}{2^{k}}\right) \phi\left(\frac{\gamma^{\prime}\left(2^{-j}\right)}{2^{n+j-k}}\right) .
$$

Based on this decomposition, we split $H_{\gamma}^{2}(f, g)$ as follows:

$$
\begin{aligned}
& \sum_{j \in \mathbb{Z}} \sum_{(m, n) \in \mathbb{Z}^{2} \backslash\left(\left(\mathbb{Z}_{-}\right)^{2} \cup \Delta\right)} \sum_{k \in \mathbb{Z}} A_{\gamma, j, m, n, k}(f, g)(x)+\sum_{j \in \mathbb{Z}} \sum_{(m, n) \in \mathbb{Z}^{2} \backslash\left(\left(\mathbb{Z}_{-}\right)^{2} \cup \Delta\right)} \sum_{k \in \mathbb{Z}} B_{\gamma, j, m, n, k}(f, g)(x) \\
= & A_{\gamma}^{2}(f, g)(x)+B_{\gamma}^{2}(f, g)(x),
\end{aligned}
$$

where

$$
\left\{\begin{array}{l}
A_{\gamma, j, m, n, k}(f, g)(x):=\int_{-\infty}^{\infty} \int_{-\infty}^{\infty} \hat{f}(\xi) \hat{g}(\eta) e^{i \xi x} e^{i \eta x} A_{j, m, n, k}(\xi, \eta) \mathrm{d} \xi \mathrm{d} \eta \\
B_{\gamma, j, m, n, k}(f, g)(x):=\int_{-\infty}^{\infty} \int_{-\infty}^{\infty} \hat{f}(\xi) \hat{g}(\eta) e^{i \xi x} e^{i \eta x} B_{j, m, n, k}(\xi, \eta) \mathrm{d} \xi \mathrm{d} \eta
\end{array}\right.
$$

Since $(m, n) \in \mathbb{Z}^{2} \backslash\left(\left(\mathbb{Z}_{-}\right)^{2} \cup \Delta\right)$, there are two cases: $n>|m|+2 / C_{1}+1$ and $m>|n|+2 / C_{1}+1$.

\subsection{Case 1: $n>|m|+2 / C_{1}+1$}

Applying the Taylor series expansion, we have

$$
\frac{1}{2^{-j} \xi+\eta 2^{-j} \gamma^{\prime}\left(2^{-j} t\right)}=\frac{1}{2^{n}} \frac{1}{\frac{\eta \gamma^{\prime}\left(2^{-j}\right)}{2^{n+j}}} \sum_{l \in \mathbb{N}} \frac{(-1)^{l}}{2^{l(n-m)}}\left[\frac{\frac{\xi}{2^{m+j}}}{\frac{\eta \gamma^{\prime}\left(2^{-j}\right)}{2^{n+j}}}\right]^{l}\left(\frac{\gamma^{\prime}\left(2^{-j}\right)}{\gamma^{\prime}\left(2^{-j} t\right)}\right)^{l+1}
$$

Then, $A_{\gamma, j, m, n, k}(f, g)(x)$ can be written as

$$
\frac{-i}{2^{n}} \sum_{l \in \mathbb{N}} \frac{(-1)^{l}}{2^{l(n-m)}} \bar{\phi}_{-l-1}\left(\frac{\gamma^{\prime}\left(2^{-j}\right)}{2^{n+j-k}}\right) \check{\bar{\phi}}_{l, m+j} * f(x) \cdot \check{\bar{\phi}}_{-l-1, k} * g(x) \cdot \int_{-\infty}^{\infty} e^{-i 2^{-j} \xi t-i \eta \gamma\left(2^{-j} t\right)} \rho^{\prime}(t)\left(\frac{\gamma^{\prime}\left(2^{-j}\right)}{\gamma^{\prime}\left(2^{-j} t\right)}\right)^{l+1} \mathrm{~d} t .
$$


Noting that $\gamma$ is either odd or even, $\gamma^{\prime}$ is increasing on $(0, \infty), \rho$ is supported on $\left\{t \in \mathbb{R}: \frac{1}{2} \leq|t| \leq\right.$ $2\}$, and by (2.3), we have

$$
\left|\int_{-\infty}^{\infty} e^{-i 2^{-j} \xi t-i \eta \gamma\left(2^{-j} t\right)} \rho^{\prime}(t)\left(\frac{\gamma^{\prime}\left(2^{-j}\right)}{\gamma^{\prime}\left(2^{-j} t\right)}\right)^{l+1} \mathrm{~d} t\right| \lesssim 2^{\frac{2 l}{C_{1}}} .
$$

By the Cauchy-Schwarz inequality,

$$
\begin{aligned}
A_{\gamma}^{2}(f, g)(x) \leq & \sum_{(m, n) \in \mathbb{Z}^{2} \backslash\left(\left(\mathbb{Z}_{-}\right)^{2} \cup \Delta\right)} \frac{1}{2^{n}} \sum_{l \in \mathbb{N}} \frac{2^{\frac{2 l}{C_{1}}}}{2^{l(n-m)}}\left[\sum_{j, k \in \mathbb{Z}}\left|\bar{\phi}_{-l-1}\left(\frac{\gamma^{\prime}\left(2^{-j}\right)}{2^{n+j-k}}\right)\right| \cdot\left|\check{\phi}_{l, m+j} * f(x)\right|^{2}\right]^{\frac{1}{2}} \\
& \times\left[\sum_{j, k \in \mathbb{Z}}\left|\bar{\phi}_{-l-1}\left(\frac{\gamma^{\prime}\left(2^{-j}\right)}{2^{n+j-k}}\right)\right| \cdot\left|\check{\phi}_{-l-1, k} * g(x)\right|^{2}\right]^{\frac{1}{2}} .
\end{aligned}
$$

For $r \geq 1$, by the triangle inequality and Hölder inequality, we bound $\left\|A_{\gamma}^{2}(f, g)\right\|_{L^{r}(\mathbb{R})}$ by

$$
\begin{aligned}
\sum_{(m, n) \in \mathbb{Z}^{2} \backslash\left(\left(\mathbb{Z}_{-}\right)^{2} \cup \Delta\right)} \frac{1}{2^{n}} \sum_{l \in \mathbb{N}} \frac{2^{\frac{2 l}{C_{1}}}}{2^{l(n-m)}}\left\|\left[\sum_{j, k \in \mathbb{Z}}\left|\bar{\phi}_{-l-1}\left(\frac{\gamma^{\prime}\left(2^{-j}\right)}{2^{n+j-k}}\right)\right| \cdot\left|\check{\bar{\phi}}_{l, m+j} * f\right|^{2}\right]^{\frac{1}{2}}\right\|_{L^{p}(\mathbb{R})} \\
\times \|\left[\left.\sum_{j, k \in \mathbb{Z}}\left|\bar{\phi}_{-l-1}\left(\frac{\gamma^{\prime}\left(2^{-j}\right)}{2^{n+j-k}}\right)\right| \cdot\left|\check{\bar{\phi}}_{-l-1, k} * g\right|^{2}\right|^{\frac{1}{2}} \|_{L^{q}(\mathbb{R})} .\right.
\end{aligned}
$$

On the other hand, from $n>|m|+2 / C_{1}+1$, it implies $\sum_{l \in \mathbb{N}} \frac{2^{\frac{2 l}{C_{1}}}}{2^{(n-m)}} \lesssim 1$. From $n>|m|+2 / C_{1}+1$, we have

$$
\sum_{(m, n) \in \mathbb{Z}^{2} \backslash\left(\left(\mathbb{Z}_{-}\right)^{2} \cup \Delta\right)} \frac{1}{2^{n}} \lesssim \sum_{(m, n) \in \mathbb{Z}^{2} \backslash\left(\left(\mathbb{Z}_{-}\right)^{2} \cup \Delta\right)} \frac{1}{2^{\frac{n}{2}}} \frac{1}{2^{\frac{|m|}{2}}} \lesssim 1 .
$$

As in (3.5) and (3.6), we assert that

$$
\left\|A_{\gamma}^{2}(f, g)\right\|_{L^{r}(\mathbb{R})} \lesssim\|f\|_{L^{p}(\mathbb{R})}\|g\|_{L^{q}(\mathbb{R})} .
$$

As in (3.9), it is easy to see that (4.11) also holds for $\frac{1}{2}<r<1$.

Applying the Taylor series expansion again, we have

$$
\frac{\eta 2^{-2 j} \gamma^{\prime \prime}\left(2^{-j} t\right)}{\left(2^{-j} \xi+\eta 2^{-j} \gamma^{\prime}\left(2^{-j} t\right)\right)^{2}}=\frac{1}{2^{n}} \frac{1}{\frac{\xi}{2^{m+j}}} \frac{2^{-j} \gamma^{\prime \prime}\left(2^{-j} t\right)}{\gamma^{\prime}\left(2^{-j}\right)} \sum_{l \in \mathbb{N}} \frac{(-1)^{l}}{2^{l(n-m)}}\left[\frac{\frac{\xi}{2^{m+j}}}{\frac{\eta \gamma^{\prime}\left(2^{-j}\right)}{2^{n+j}}}\right]^{l+1}\left(\frac{\gamma^{\prime}\left(2^{-j}\right)}{\gamma^{\prime}\left(2^{-j} t\right)}\right)^{l+1} .
$$

We can then write $B_{\gamma, j, m, n}(f, g)(x)$ as

$$
\frac{i}{2^{n}} \sum_{l \in \mathbb{N}} \frac{(-1)^{l}}{2^{l(n-m)}} \bar{\phi}_{-l-1}\left(\frac{\gamma^{\prime}\left(2^{-j}\right)}{2^{n+j-k}}\right) \check{\bar{\phi}}_{l, m+j} * f(x) \cdot \check{\bar{\phi}}_{-l-1, k} * g(x)
$$




$$
\times \int_{-\infty}^{\infty} e^{-i 2^{-j} \xi t-i \eta \gamma\left(2^{-j} t\right)} \frac{2^{-j} \gamma^{\prime \prime}\left(2^{-j} t\right)}{\gamma^{\prime}\left(2^{-j}\right)}\left(\frac{\gamma^{\prime}\left(2^{-j}\right)}{\gamma^{\prime}\left(2^{-j} t\right)}\right)^{l+1} \rho(t) \mathrm{d} t
$$

Since $\gamma$ is either odd or even, $\gamma^{\prime}$ is increasing on $(0, \infty), \rho$ is supported on $\left\{t \in \mathbb{R}: \frac{1}{2} \leq|t| \leq 2\right\}$, and by (2.1) and (2.3), it is easy to see that $\left|\frac{2^{-j} \gamma^{\prime \prime}\left(2^{-j} t\right)}{\gamma^{\prime}\left(2^{-j}\right)}\right|=\left|\frac{2^{-j} t \gamma^{\prime \prime}\left(2^{-j} t\right)}{\gamma^{\prime}\left(2^{-j} t\right)} \frac{\gamma^{\prime}\left(2^{-j} t\right)}{\gamma^{\prime}\left(2^{-j}\right)} \frac{1}{t}\right| \lesssim 1$. Thus

$$
\left|\int_{-\infty}^{\infty} e^{-i 2^{-j} \xi t-i \eta \gamma\left(2^{-j} t\right)} \frac{2^{-j} \gamma^{\prime \prime}\left(2^{-j} t\right)}{\gamma^{\prime}\left(2^{-j}\right)}\left(\frac{\gamma^{\prime}\left(2^{-j}\right)}{\gamma^{\prime}\left(2^{-j} t\right)}\right)^{l+1} \rho(t) \mathrm{d} t\right| \lesssim 2^{\frac{2 l}{C_{1}}} .
$$

As in (4.9) and (4.10), for $r>\frac{1}{2}$, we assert that

$$
\left\|B_{\gamma}^{2}(f, g)\right\|_{L^{r}(\mathbb{R})} \lesssim\|f\|_{L^{p}(\mathbb{R})}\|g\|_{L^{q}(\mathbb{R})} .
$$

\subsection{Case 2: $m>|n|+2 / C_{1}+1$}

By the Taylor series expansion, we have

$$
\frac{1}{2^{-j} \xi+\eta 2^{-j} \gamma^{\prime}\left(2^{-j} t\right)}=\frac{1}{2^{m}} \frac{1}{\frac{\xi}{2^{m+j}}} \sum_{l \in \mathbb{N}} \frac{(-1)^{l}}{2^{l(m-n)}}\left[\frac{\frac{\eta \gamma^{\prime}\left(2^{-j}\right)}{2^{n+j}}}{\frac{\xi}{2^{m+j}}}\right]^{l}\left(\frac{\gamma^{\prime}\left(2^{-j} t\right)}{\gamma^{\prime}\left(2^{-j}\right)}\right)^{l} .
$$

As in case 1, we have

$$
\left\|A_{\gamma}^{2}(f, g)\right\|_{L^{r}(\mathbb{R})} \lesssim\|f\|_{L^{p}(\mathbb{R})}\|g\|_{L^{q}(\mathbb{R})}
$$

for $r>\frac{1}{2}$. Furthermore,

$$
\frac{\eta 2^{-2 j} \gamma^{\prime \prime}\left(2^{-j} t\right)}{\left(2^{-j} \xi+\eta 2^{-j} \gamma^{\prime}\left(2^{-j} t\right)\right)^{2}}=\frac{1}{2^{m}} \frac{1}{\frac{\xi}{2^{m+j}}} \frac{2^{-j} \gamma^{\prime \prime}\left(2^{-j} t\right)}{\gamma^{\prime}\left(2^{-j} t\right)} \sum_{l \in \mathbb{N}} \frac{(-1)^{l}}{2^{(l+1)(m-n)}}\left[\frac{\frac{\eta \gamma^{\prime}\left(2^{-j}\right)}{2^{n+j}}}{\frac{\xi}{2^{m+j}}}\right]^{l+1}\left(\frac{\gamma^{\prime}\left(2^{-j} t\right)}{\gamma^{\prime}\left(2^{-j}\right)}\right)^{l+1}
$$

As in case 1 , for $r>\frac{1}{2}$, again we have

$$
\left\|B_{\gamma}^{2}(f, g)\right\|_{L^{r}(\mathbb{R})} \lesssim\|f\|_{L^{p}(\mathbb{R})}\|g\|_{L^{q}(\mathbb{R})} .
$$

From (4.4), 4.11), 4.14, 4.16) and 4.18), we may obtain

$$
\left\|H_{\gamma}^{2}(f, g)\right\|_{L^{r}(\mathbb{R})} \lesssim\|f\|_{L^{p}(\mathbb{R})}\|g\|_{L^{q}(\mathbb{R})}
$$

for $r>\frac{1}{2}$. This is the desired estimate for the second item $H_{\gamma}^{2}(f, g)$.

\section{The $L^{2}(\mathbb{R}) \times L^{2}(\mathbb{R}) \rightarrow L^{1}(\mathbb{R})$ boundedness of $H_{\gamma}^{3}(f, g)$}

Note that $\Delta=\left\{(m, n) \in \mathbb{Z}^{2}: m, n \geq 0,|m-n| \leq 2 / C_{1}+1\right\}$ and $m_{j}^{3}=\sum_{(m, n) \in \Delta, k \in \mathbb{Z}} m_{j, m, n, k} ;$ without loss of generality, we may write that

$$
m_{j}^{3}=\sum_{m \in \mathbb{N}} \sum_{k \in \mathbb{Z}} m_{j, m, k}
$$


where $m_{j, m, k}:=m_{j, m, m, k}$. Therefore, we rewrite $H_{\gamma}^{3}(f, g)$ as

$$
H_{\gamma}^{3}(f, g)(x)=\sum_{j \in \mathbb{Z}} \sum_{m \in \mathbb{N}} \sum_{k \in \mathbb{Z}} H_{j, m, k}(f, g)(x) .
$$

where

$$
H_{j, m, k}(f, g)(x):=\phi\left(\frac{\gamma^{\prime}\left(2^{-j}\right)}{2^{m+j-k}}\right) \int_{-\infty}^{\infty} \int_{-\infty}^{\infty} \hat{f}(\xi) \hat{g}(\eta) e^{i \xi x} e^{i \eta x} m_{j}(\xi, \eta) \phi\left(\frac{\xi}{2^{m+j}}\right) \phi\left(\frac{\eta}{2^{k}}\right) \mathrm{d} \xi \mathrm{d} \eta
$$

with $m_{j}$ as in (2.8).

We observe that in order to obtain

$$
\left\|H_{\gamma}^{3}(f, g)\right\|_{L^{1}(\mathbb{R})} \lesssim\|f\|_{L^{2}(\mathbb{R})}\|g\|_{L^{2}(\mathbb{R})},
$$

it suffices to prove the following Proposition 5.1. Indeed, notice that $\phi$ is a standard bump function supported on $\left\{t \in \mathbb{R}: \frac{1}{2} \leq|t| \leq 2\right\}$; let $\Phi$ be a bump function supported on $\left\{t \in \mathbb{R}: \frac{1}{8} \leq|t| \leq 8\right\}$ such that $\Phi(t)=1$ on $\left\{t \in \mathbb{R}: \frac{1}{4} \leq|t| \leq 4\right\}$; thus, it is safe to insert $\Phi$ into $H_{j, m, k}(f, g)$. In other words, recall that $\psi_{\lambda}(\xi)=2^{\lambda} \psi\left(2^{\lambda} \xi\right)$, we have

$$
H_{j, m, k}(f, g)(x)=\Phi\left(\frac{\gamma^{\prime}\left(2^{-j}\right)}{2^{m+j-k}}\right) H_{j, m, k}\left(\check{\Phi}_{m+j} * f, \check{\Phi}_{k} * g\right)(x) .
$$

By the triangle inequality, the Cauchy-Schwarz inequality and (5.6), it implies that $\left\|H_{\gamma}^{3}(f, g)\right\|_{L^{1}(\mathbb{R})}$ can be bounded by

$$
\sum_{m \in \mathbb{N}} 2^{-\varepsilon_{0} m}\left[\sum_{j, k \in \mathbb{Z}}\left|\Phi\left(\frac{\gamma^{\prime}\left(2^{-j}\right)}{2^{m+j-k}}\right)\right| \cdot\left\|\check{\Phi}_{m+j} * f\right\|_{L^{2}(\mathbb{R})}^{2}\right]^{\frac{1}{2}} \cdot\left[\sum_{j, k \in \mathbb{Z}}\left|\Phi\left(\frac{\gamma^{\prime}\left(2^{-j}\right)}{2^{m+j-k}}\right)\right| \cdot\left\|\check{\Phi}_{k} * g\right\|_{L^{2}(\mathbb{R})}^{2}\right]^{\frac{1}{2}} .
$$

By this estimate with (3.4), (3.5) and the Littlewood-Paley theory, we may obtain (5.4).

Proposition 5.1. There exist positive constants $C$ and $\varepsilon_{0}$ such that

$$
\left\|H_{j, m, k}(f, g)\right\|_{L^{1}(\mathbb{R})} \leq C 2^{-\varepsilon_{0} m}\|f\|_{L^{2}(\mathbb{R})}\|g\|_{L^{2}(\mathbb{R})}
$$

holds uniformly for $j, k \in \mathbb{Z}$.

As in [34], we define the bilinear operator $B_{j, m, k}(f, g)(x)$ as

$$
2^{\frac{m+j-k}{2}} \phi\left(\frac{\gamma^{\prime}\left(2^{-j}\right)}{2^{m+j-k}}\right) \int_{-\infty}^{\infty} \check{\phi} * f\left(2^{m+j-k} x-2^{m} t\right) \check{\phi} * g\left(x-2^{k} \gamma\left(2^{-j} t\right)\right) \rho(t) \mathrm{d} t \quad \text { if } \quad j \geq 0 \text {; }
$$

and

$$
2^{\frac{k-m-j}{2}} \phi\left(\frac{\gamma^{\prime}\left(2^{-j}\right)}{2^{m+j-k}}\right) \int_{-\infty}^{\infty} \check{\phi} * f\left(x-2^{m} t\right) \check{\phi} * g\left(2^{k-m-j} x-2^{k} \gamma\left(2^{-j} t\right)\right) \rho(t) \mathrm{d} t \quad \text { if } \quad j<0 .
$$

We observe that (5.6) is equivalent to

$$
\left\|B_{j, m, k}(f, g)\right\|_{L^{1}(\mathbb{R})} \lesssim 2^{-\varepsilon_{0} m}\|f\|_{L^{2}(\mathbb{R})}\|g\|_{L^{2}(\mathbb{R})} .
$$


Indeed, let $\xi:=2^{m+j} \xi$ and $\eta:=2^{k} \eta$; we then write $H_{j, m, k}(f, g)(x)$ as

$2^{m+j+k} \phi\left(\frac{\gamma^{\prime}\left(2^{-j}\right)}{2^{m+j-k}}\right) \int_{-\infty}^{\infty} \int_{-\infty}^{\infty} \hat{f}\left(2^{m+j} \xi\right) \hat{g}\left(2^{k} \eta\right) e^{i 2^{m+j} \xi x} e^{i 2^{k} \eta x}\left(\int_{-\infty}^{\infty} e^{-i 2^{m} \xi t} e^{-i 2^{k} \eta \gamma\left(2^{-j} t\right)} \rho(t) \mathrm{d} t\right) \phi(\xi) \phi(\eta) \mathrm{d} \xi \mathrm{d} \eta$.

For $j \geq 0$, let $x:=2^{-k} x$; we may therefore write

$$
\begin{aligned}
2^{-k} H_{j, m, k}(f, g)\left(2^{-k} x\right)= & 2^{\frac{m+j-k}{2}} \phi\left(\frac{\gamma^{\prime}\left(2^{-j}\right)}{2^{m+j-k}}\right) \int_{-\infty}^{\infty} \int_{-\infty}^{\infty} 2^{\frac{m+j}{2}} \hat{f}\left(2^{m+j} \xi\right) 2^{\frac{k}{2}} \hat{g}\left(2^{k} \eta\right) e^{i 2^{m+j-k} \xi x} e^{i \eta x} \\
& \times\left(\int_{-\infty}^{\infty} e^{-i 2^{m} \xi t} e^{-i 2^{k} \eta \gamma\left(2^{-j} t\right)} \rho(t) \mathrm{d} t\right) \phi(\xi) \phi(\eta) \mathrm{d} \xi \mathrm{d} \eta .
\end{aligned}
$$

Note that $\left\|2^{\frac{m+j}{2}} \hat{f}\left(2^{m+j} \cdot\right)\right\|_{L^{2}(\mathbb{R})}=\|\hat{f}\|_{L^{2}(\mathbb{R})}$ and $\left\|2^{\frac{k}{2}} \hat{g}\left(2^{k} \cdot\right)\right\|_{L^{2}(\mathbb{R})}=\|\hat{g}\|_{L^{2}(\mathbb{R})}$; we conclude that (5.6) is equivalent to (5.9), where $B_{j, m, k}(f, g)$ is defined in (5.7). For $j<0$, the only difference is to make the variable change $x:=\frac{x}{2^{m+j}}$, and we omit the details.

We now turn to the proof of [5.9). In what follows, we will only focus on the first case, i.e., $j \geq 0$, since the second case, i.e., $j<0$, is similar. From Proposition 2.2 and (5.9), we can assume that $j$ and $m$ are sufficiently large.

Claim: (5.9) is equivalent to Proposition 5.2 below.

Proof of the Claim. This claim is essentially [34, Lemma 5.1]. As in [34], let $\psi$ be a nonnegative Schwartz function such that $\hat{\psi}$ is supported on $\left\{t \in \mathbb{R}:|t| \leq \frac{1}{100}\right\}$ and satisfies $\hat{\psi}(0)=1$. Then, $B_{j, m, k}(f, g)(x)$ can be written as

$$
\begin{aligned}
& 2^{\frac{m+j-k}{2}} \phi\left(\frac{\gamma^{\prime}\left(2^{-j}\right)}{2^{m+j-k}}\right) \sum_{N \in \mathbb{Z}} \sum_{k_{1}, k_{2} \in \mathbb{Z}} \int_{-\infty}^{\infty}\left(\chi_{\left[2^{m}\left(N+k_{1}\right), 2^{m}\left(N+k_{1}+1\right)\right]} * \psi_{-m} \cdot \check{\phi} * f\right)\left(2^{m+j-k} x-2^{m} t\right) \\
& \times\left(\chi_{\left[2^{k-j}\left(N+k_{2}\right), 2^{k-j}\left(N+k_{2}+1\right)\right]} * \psi_{j-k} \cdot \check{\phi} * g\right)\left(x-2^{k} \gamma\left(2^{-j} t\right)\right) \cdot \rho(t) \mathrm{d} t \cdot \chi_{\left[2^{k-j} N, 2^{k-j}(N+1)\right]}(x) .
\end{aligned}
$$

We split

$$
B_{j, m, k}(f, g)(x):=B_{j, m, k}^{I}(f, g)(x)+B_{j, m, k}^{I I}(f, g)(x),
$$

where $B_{j, m, k}^{I}(f, g)$ sums over $A:=\left\{k_{1}, k_{2} \in \mathbb{Z}: \max \left\{\left|k_{1}\right|,\left|k_{2}\right|\right\} \geq \Theta\right\}$ and $B_{j, m, k}^{I I}(f, g)$ sums over $B:=$ $\left\{k_{1}, k_{2} \in \mathbb{Z}: \max \left\{\left|k_{1}\right|,\left|k_{2}\right|\right\}<\Theta\right\}$, where $\Theta:=2^{\frac{\varepsilon_{0}^{\prime}}{3} m}$ is sufficiently large.

For $B_{j, m, k}^{I}(f, g)$, we have $\left|\left(\chi_{\left[2^{m}\left(N+k_{1}\right), 2^{m}\left(N+k_{1}+1\right)\right]} * \psi_{-m}\right)\left(2^{m+j-k} x-2^{m} t\right)\right| \lesssim \frac{1}{\left|k_{1}\right|^{3}}$. Note that $\frac{\gamma(t)}{t}$ is increasing on $(0, \infty)$; it implies $2^{j} \gamma\left(2^{-j} t\right)$ is sufficiently small if $j$ is sufficiently large, and we also have $\left|\left(\chi_{\left[2^{k-j}\left(N+k_{2}\right), 2^{k-j}\left(N+k_{2}+1\right)\right]} * \psi_{j-k}\right)\left(x-2^{k} \gamma\left(2^{-j} t\right)\right)\right| \lesssim \frac{1}{\left|k_{2}\right|^{3}}$. Thus, $B_{j, m, k}^{I}(f, g)(x)$ is bounded by

$$
\begin{aligned}
& 2^{\frac{m+j-k}{2}} \phi\left(\frac{\gamma^{\prime}\left(2^{-j}\right)}{2^{m+j-k}}\right) \sum_{N \in \mathbb{Z}} \sum_{k_{1}, k_{2} \in A} \frac{1}{\left|k_{1}\right|^{3}} \frac{1}{\left|k_{2}\right|^{3}} \int_{-\infty}^{\infty}\left|\check{\phi} * f\left(2^{m+j-k} x-2^{m} t\right)\right| \\
& \times\left|\check{\phi} * g\left(x-2^{k} \gamma\left(2^{-j} t\right)\right)\right| \cdot|\rho(t)| \mathrm{d} t \cdot \chi_{\left[2^{k-j} N, 2^{k-j}(N+1)\right]}(x) \\
\lesssim & \frac{1}{\Theta} 2^{\frac{m+j-k}{2}} \phi\left(\frac{\gamma^{\prime}\left(2^{-j}\right)}{2^{m+j-k}}\right) \int_{-\infty}^{\infty}\left|\check{\phi} * f\left(2^{m+j-k} x-2^{m} t\right) \check{\phi} * g\left(x-2^{k} \gamma\left(2^{-j} t\right)\right)\right| \cdot|\rho(t)| \mathrm{d} t .
\end{aligned}
$$


The last inequality is a result of the fact that $\sum_{|k| \geq \Theta} \frac{1}{|k|^{3}} \lesssim \frac{1}{\Theta}$ and $\sum_{k \in \mathbb{Z}} \frac{1}{|k|^{3}} \lesssim 1$. By the Hölder and Young inequalities, it now follows that

$$
\left\|B_{j, m, k}^{I}(f, g)\right\|_{L^{1}(\mathbb{R})} \lesssim \frac{1}{\Theta}\|f\|_{L^{2}(\mathbb{R})}\|g\|_{L^{2}(\mathbb{R})} .
$$

For $B_{j, m, k}^{I I}(f, g)$, let $j$ and $m$ be sufficiently large; we have that

$$
\mathcal{F}\left(\chi_{\left[2^{m}\left(N+k_{1}\right), 2^{m}\left(N+k_{1}+1\right)\right]} * \psi_{-m} \cdot \check{\phi} * f\right) \quad \text { and } \mathcal{F}\left(\chi_{\left[2^{k-j}\left(N+k_{2}\right), 2^{k-j}\left(N+k_{2}+1\right)\right]} * \psi_{j-k} \cdot \check{\phi} * g\right)
$$

are supported on $\left\{t \in \mathbb{R}: \frac{1}{4} \leq|t| \leq 4\right\}$, where $\mathcal{F}(f)$ means the Fourier transform of $f$. Then, $B_{j, m, k}^{I I}(f, g)(x)$ can be written as

$$
\begin{aligned}
& \sum_{N \in \mathbb{Z}} \sum_{k_{1}, k_{2} \in B} B_{j, m, k}^{*}\left(\chi_{\left[2^{m}\left(N+k_{1}\right), 2^{m}\left(N+k_{1}+1\right)\right]} * \psi_{-m} \cdot \check{\phi} * f, \chi_{\left[2^{k-j}\left(N+k_{2}\right), 2^{k-j}\left(N+k_{2}+1\right)\right]} * \psi_{j-k} \cdot \check{\phi} * g\right)(x) \\
& \times \chi_{\left[2^{k-j} N, 2^{k-j}(N+1)\right]}(x) .
\end{aligned}
$$

The definition of $B_{j, m, k}^{*}(f, g)$ will be given in Proposition 5.2. From $\sum_{N \in \mathbb{Z}} \chi_{\left[2^{m}\left(N+k_{1}\right), 2^{m}\left(N+k_{1}+1\right)\right]} *$ $\left.\psi_{-m}\right|^{2} \lesssim\|\psi\|_{L^{1}(\mathbb{R})}^{2}$ and $\sum_{N \in \mathbb{Z}}\left|\chi_{\left[2^{k-j}\left(N+k_{2}\right), 2^{k-j}\left(N+k_{2}+1\right)\right]} * \psi_{j-k}\right|^{2} \lesssim\|\psi\|_{L^{1}(\mathbb{R})}^{2}$, by the Cauchy-Schwarz inequality and the Young inequality, (5.15), $\left\|B_{j, m, k}^{I I}(f, g)\right\|_{L^{1}(\mathbb{R})}$ can be bounded by

$$
\begin{aligned}
& \Theta^{2} 2^{-\varepsilon_{0}^{\prime} m}\left[\sum_{N \in \mathbb{Z}}\left\|\chi_{\left[2^{m}\left(N+k_{1}\right), 2^{m}\left(N+k_{1}+1\right)\right]} * \psi_{-m} \cdot \check{\phi} * f\right\|_{L^{2}(\mathbb{R})}^{2}\right]^{\frac{1}{2}} \\
& \times\left[\sum_{N \in \mathbb{Z}}\left\|\chi_{\left[2^{k-j}\left(N+k_{2}\right), 2^{k-j}\left(N+k_{2}+1\right)\right]} * \psi_{j-k} \cdot \check{\phi} * g\right\|_{L^{2}(\mathbb{R})}^{2}\right]^{\frac{1}{2}} \lesssim \Theta^{2} 2^{-\varepsilon_{0}^{\prime} m}\|f\|_{L^{2}(\mathbb{R})}\|g\|_{L^{2}(\mathbb{R})} .
\end{aligned}
$$

From (5.12) and (5.13), note that $\Theta=2^{\frac{\varepsilon_{0}^{\prime}}{3} m}$; we obtain

$$
\left\|B_{j, m, k}(f, g)\right\|_{L^{1}(\mathbb{R})} \lesssim 2^{-\frac{\varepsilon_{0}^{\prime}}{3} m}\|f\|_{L^{2}(\mathbb{R})}\|g\|_{L^{2}(\mathbb{R})} .
$$

This is (5.9) if we let $\varepsilon_{0}:=\frac{\varepsilon_{0}^{\prime}}{3}$.

Proposition 5.2. There exist positive constants $C$ and $\varepsilon_{0}^{\prime}$ such that

$$
\left\|B_{j, m, k}^{*}(f, g) \cdot \chi_{\left[2^{k-j} N, 2^{k-j}(N+1)\right]}\right\|_{L^{1}(\mathbb{R})} \leq C 2^{-\varepsilon_{0}^{\prime} m}\|f\|_{L^{2}(\mathbb{R})}\|g\|_{L^{2}(\mathbb{R})}
$$

holds uniformly for $j \in \mathbb{Z}$ and $N \in \mathbb{Z}$, where $B_{j, m, k}^{*}(f, g)$ is defined as

$$
B_{j, m, k}^{*}(f, g)(x):=2^{\frac{m+j-k}{2}} \phi\left(\frac{\gamma^{\prime}\left(2^{-j}\right)}{2^{m+j-k}}\right) \int_{-\infty}^{\infty} \check{\Phi} * f\left(2^{m+j-k} x-2^{m} t\right) \check{\Phi} * g\left(x-2^{k} \gamma\left(2^{-j} t\right)\right) \rho(t) d t
$$

and $\Phi$ is the same bump function as in (5.5).

We will prove Proposition 5.2 in three steps. 


\subsection{An estimate by using $T T^{*}$ argument}

In this subsection, we show that

$$
\left\|B_{j, m, k}^{*}(f, g) \cdot \chi_{\left[2^{k-j} N, 2^{k-j}(N+1)\right]}\right\|_{L^{1}(\mathbb{R})} \lesssim 2^{-\frac{2 m+j-k}{6}}\|f\|_{L^{2}(\mathbb{R})}\|g\|_{L^{2}(\mathbb{R})} .
$$

By the Hölder inequality, it suffices to show that

$$
\left\|B_{j, m, k}^{*}(f, g)\right\|_{L^{2}(\mathbb{R})} \lesssim 2^{-\frac{2 m+j-k}{6}} 2^{\frac{j-k}{2}}\|f\|_{L^{2}(\mathbb{R})}\|g\|_{L^{2}(\mathbb{R})} .
$$

We rewrite $B_{j, m, k}^{*}(f, g)(x)$ as

$$
2^{\frac{m+j-k}{2}} \phi\left(\frac{\gamma^{\prime}\left(2^{-j}\right)}{2^{m+j-k}}\right) \int_{-\infty}^{\infty} \int_{-\infty}^{\infty} \hat{f}(\xi) \hat{g}(\eta) e^{i 2^{m+j-k} \xi x} e^{i \eta x} I_{j, m, k}(\xi, \eta) \Phi(\xi) \Phi(\eta) \mathrm{d} \xi \mathrm{d} \eta,
$$

where

$$
I_{j, m, k}(\xi, \eta):=\int_{-\infty}^{\infty} e^{-i 2^{m} \xi t} e^{-i 2^{k} \eta \gamma\left(2^{-j} t\right)} \rho(t) \mathrm{d} t
$$

Let

$$
\varphi(t, \xi, \eta):=-\xi t-2^{k-m} \eta \gamma\left(2^{-j} t\right) .
$$

We denote $\varphi_{1}^{\prime}$ as the derivative of $\varphi$ with respect to the first variable $t$. Then

$$
\varphi_{1}^{\prime}(t, \xi, \eta)=-\xi-2^{k-m-j} \gamma^{\prime}\left(2^{-j} t\right) \eta \quad \text { and } \quad \varphi_{1}^{\prime \prime}(t, \xi, \eta)=-2^{k-m-2 j} \gamma^{\prime \prime}\left(2^{-j} t\right) \eta .
$$

We define $t_{0}(\xi, \eta)$ as

$$
\varphi_{1}^{\prime}\left(t_{0}(\xi, \eta), \xi, \eta\right)=0
$$

For simplicity, we may further assume that $\frac{1}{2} \leq\left|t_{0}(\xi, \eta)\right| \leq 2$. By the method of the stationary phase, we assert that

$$
I_{j, m, k}(\xi, \eta)=e^{i 2^{m} \varphi\left(t_{0}(\xi, \eta), \xi, \eta\right)}\left(\frac{2 \pi}{-i 2^{m} \varphi_{1}^{\prime \prime}\left(t_{0}(\xi, \eta), \xi, \eta\right)}\right)^{\frac{1}{2}} \rho\left(t_{0}(\xi, \eta)\right)+O\left(2^{-\frac{3}{2} m}\right) .
$$

For $h \in L^{2}(\mathbb{R})$, we have $\int_{-\infty}^{\infty} B_{j, m, k}^{*}(f, g)(x) h(x) \mathrm{d} x$ is equal to

$$
2^{\frac{m+j-k}{2}} \phi\left(\frac{\gamma^{\prime}\left(2^{-j}\right)}{2^{m+j-k}}\right) \int_{-\infty}^{\infty} \int_{-\infty}^{\infty} \hat{f}(\xi) \Phi(\xi) \hat{g}(\eta) \Phi(\eta) I_{j, m, k}(\xi, \eta) \breve{h}\left(2^{m+j-k} \xi+\eta\right) \mathrm{d} \xi \mathrm{d} \eta .
$$

Based on (5.23), we estimate $\int_{-\infty}^{\infty} B_{j, m, k}^{*}(f, g)(x) h(x) \mathrm{d} x$ by considering the following two parts:

Part A: $O\left(2^{-\frac{3}{2} m}\right)$

With some abuse of notation, we write $\int_{-\infty}^{\infty} B_{j, m, k}^{*}(f, g)(x) h(x) \mathrm{d} x$ as

$$
2^{\frac{m+j-k}{2}} \phi\left(\frac{\gamma^{\prime}\left(2^{-j}\right)}{2^{m+j-k}}\right) \int_{-\infty}^{\infty} \int_{-\infty}^{\infty} \hat{f}(\xi) \Phi(\xi) \hat{g}(\eta) \Phi(\eta) \breve{h}\left(2^{m+j-k} \xi+\eta\right) \cdot O\left(2^{-\frac{3}{2} m}\right) \mathrm{d} \xi \mathrm{d} \eta .
$$


Thus, by the Hölder inequality and Plancherel's formula, we bound | $\int_{-\infty}^{\infty} B_{j, m, k}^{*}(f, g)(x) h(x) \mathrm{d} x \mid$ by $2^{-\frac{3}{2} m} 2^{\frac{m+j-k}{2}} \int_{-\infty}^{\infty}|\hat{f}(\xi) \Phi(\xi)| \int_{-\infty}^{\infty}\left|\hat{g}(\eta) \Phi(\eta) \check{h}\left(2^{m+j-k} \xi+\eta\right)\right| \mathrm{d} \eta \mathrm{d} \xi \lesssim 2^{-\frac{3}{2} m} 2^{\frac{m+j-k}{2}}\|f\|_{L^{2}(\mathbb{R})}\|g\|_{L^{2}(\mathbb{R})}\|h\|_{L^{2}(\mathbb{R})}$.

Furthermore, let $h:=\operatorname{sgn}\left(B_{j, m, k}^{*}(f, g)\right) \cdot \chi_{\left[2^{k-j} N, 2^{k-j}(N+1)\right]}$; we have

$$
\left\|B_{j, m, k}^{*}(f, g) \cdot \chi_{\left[2^{k-j} N, 2^{k-j}(N+1)\right]}\right\|_{L^{1}(\mathbb{R})} \lesssim 2^{-m}\|f\|_{L^{2}(\mathbb{R})}\|g\|_{L^{2}(\mathbb{R})} .
$$

This is (5.15) as desired.

Part B: $e^{i 2^{m} \varphi\left(t_{0}(\xi, \eta), \xi, \eta\right)}\left(\frac{2 \pi}{-i 2^{m} \varphi_{1}^{\prime \prime}\left(t_{0}(\xi, \eta), \xi, \eta\right)}\right)^{\frac{1}{2}} \rho\left(t_{0}(\xi, \eta)\right)$

From (2.1) and (2.3), we have $\left|\frac{2^{-j} \gamma^{\prime \prime}\left(2^{-j} t\right)}{\gamma^{\prime}\left(2^{-j}\right)}\right|=\left|\frac{2^{-j} t \gamma^{\prime \prime}\left(2^{-j} t\right)}{\gamma^{\prime}\left(2^{-j} t\right)} \frac{\gamma^{\prime}\left(2^{-j} t\right)}{\gamma^{\prime}\left(2^{-j}\right)} \frac{1}{t}\right| \approx 1$. For simplicity, we write $\int_{-\infty}^{\infty} B_{j, m, k}^{*}(f, g)(x) h(x) \mathrm{d} x$ as

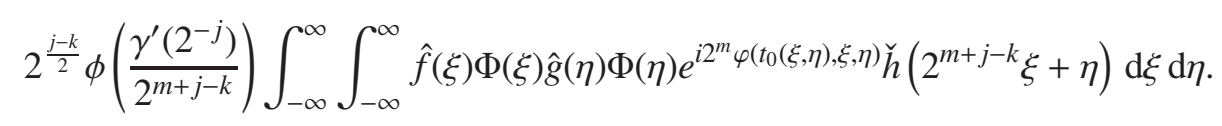

Changing variables $\xi:=\frac{\xi-\eta}{2^{m+j-k}}$ and $\eta:=2^{m+j-k} \eta$, we have

$$
\begin{aligned}
& 2^{\frac{j-k}{2}} \phi\left(\frac{\gamma^{\prime}\left(2^{-j}\right)}{2^{m+j-k}}\right) \int_{-\infty}^{\infty} \int_{-\infty}^{\infty}(\hat{f} \Phi)\left(\frac{\xi}{2^{m+j-k}}-\eta\right)(\hat{g} \Phi)\left(2^{m+j-k} \eta\right) \\
\times & e^{i 2^{m} \varphi\left(t_{0}\left(\frac{\xi}{2^{m+j-k}}-\eta, 2^{m+j-k} \eta\right), \frac{\xi}{2^{m+j-k}}-\eta, 2^{m+j-k} \eta\right) \breve{h}(\xi) \mathrm{d} \xi \mathrm{d} \eta .}
\end{aligned}
$$

From the Hölder inequality and Plancherel formula, it can be bounded by $2^{\frac{j-k}{2}}\left\|T_{j, m, k}(f, g)\right\|_{L^{2}(\mathbb{R})}\|h\|_{L^{2}(\mathbb{R})}$, where $T_{j, m, k}(f, g)(\xi)$ is defined as

$$
\phi\left(\frac{\gamma^{\prime}\left(2^{-j}\right)}{2^{m+j-k}}\right) \int_{-\infty}^{\infty}(\hat{f} \Phi)\left(\frac{\xi}{2^{m+j-k}}-\eta\right)(\hat{g} \Phi)\left(2^{m+j-k} \eta\right) e^{i 2^{m} \varphi\left(t_{0}\left(\frac{\xi}{2^{m+j-k}}-\eta, 2^{m+j-k} \eta\right), \frac{\xi}{2^{m+j-k}}-\eta, 2^{m+j-k} \eta\right)} \mathrm{d} \eta .
$$

Therefore, 5.17 can be reduced to

$$
\left\|T_{j, m, k}(f, g)\right\|_{L^{2}(\mathbb{R})} \lesssim 2^{-\frac{2 m+k+j}{6}}\|f\|_{L^{2}(\mathbb{R})}\|g\|_{L^{2}(\mathbb{R})} .
$$

By the $T T^{*}$ argument, we obtain that $\left\|T_{j, m, k}(f, g)\right\|_{L^{2}(\mathbb{R})}^{2}$ equals to

$$
\begin{array}{r}
\int_{-\infty}^{\infty}\left[\int_{-\infty}^{\infty} \int_{-\infty}^{\infty} \phi^{2}\left(\frac{\gamma^{\prime}\left(2^{-j}\right)}{2^{m+j-k}}\right) F\left(\xi, \eta_{1}, \eta_{2}\right) G\left(\xi, \eta_{1}, \eta_{2}\right) e^{i 2^{m} \varphi\left(t_{0}\left(\frac{\xi}{2^{m+j-k}}-\eta_{1}, 2^{m+j-k} \eta_{1}\right), \frac{\xi}{2^{m+j-k}}-\eta_{1}, 2^{m+j-k} \eta_{1}\right)}\right. \\
\left.\times e^{-i 2^{m} \varphi\left(t_{0}\left(\frac{\xi}{2^{m+j-k}}-\eta_{2}, 2^{m+j-k} \eta_{2}\right), \frac{\xi}{2^{m+j-k}}-\eta_{2}, 2^{m+j-k} \eta_{2}\right)} \mathrm{d} \eta_{1} \mathrm{~d} \eta_{2}\right] \mathrm{d} \xi,
\end{array}
$$

where

$$
\left\{\begin{array}{l}
F\left(\xi, \eta_{1}, \eta_{2}\right):=(\hat{f} \Phi)\left(\frac{\xi}{2^{m+j-k}}-\eta_{1}\right) \overline{(\hat{f} \Phi)\left(\frac{\xi}{2^{m+j-k}}-\eta_{2}\right)} \\
G\left(\xi, \eta_{1}, \eta_{2}\right):=(\hat{g} \Phi)\left(2^{m+j-k} \eta_{1}\right) \overline{(\hat{g} \Phi)\left(2^{m+j-k} \eta_{2}\right)}
\end{array}\right.
$$


Let $\eta_{1}:=\eta$ and $\eta_{2}:=\eta+\tau$; then, $\left\|T_{j, m, k}(f, g)\right\|_{L^{2}(\mathbb{R})}^{2}$ is equal to

$$
\begin{gathered}
\int_{-\infty}^{\infty}\left[\int_{-\infty}^{\infty} \int_{-\infty}^{\infty} \phi^{2}\left(\frac{\gamma^{\prime}\left(2^{-j}\right)}{2^{m+j-k}}\right) F_{\tau}\left(\frac{\xi}{2^{m+j-k}}-\eta\right) G_{\tau}\left(2^{m+j-k} \eta\right) e^{i 2^{m} \varphi\left(t_{0}\left(\frac{\xi}{2^{m+j-k}}-\eta, 2^{m+j-k} \eta\right), \frac{\xi}{2^{m+j-k}}-\eta, 2^{m+j-k} \eta\right)}\right. \\
\left.\times e^{-i 2^{m} \varphi\left(t_{0}\left(\frac{\xi}{2^{m+j-k}}-\eta-\tau, 2^{m+j-k}(\eta+\tau)\right), \frac{\xi}{2^{m+j-k}}-\eta-\tau, 2^{m+j-k}(\eta+\tau)\right)} \mathrm{d} \xi \mathrm{d} \eta\right] \mathrm{d} \tau,
\end{gathered}
$$

where $F_{\tau}(\cdot):=(\hat{f} \Phi)(\cdot) \overline{(\hat{f} \Phi)(\cdot-\tau)}$ and $G_{\tau}(\cdot):=(\hat{g} \Phi)(\cdot) \overline{(\hat{g} \Phi)\left(\cdot+2^{m+j-k} \tau\right)}$. Furthermore, let $u:=$ $\frac{\xi}{2^{m+j-k}}-\eta$ and $v:=2^{m+j-k} \eta$; we have

$$
\left\|T_{j, m, k}(f, g)\right\|_{L^{2}(\mathbb{R})}^{2}=\phi^{2}\left(\frac{\gamma^{\prime}\left(2^{-j}\right)}{2^{m+j-k}}\right) \int_{-\infty}^{\infty}\left(\int_{-\infty}^{\infty} \int_{-\infty}^{\infty} F_{\tau}(u) G_{\tau}(v) e^{i 2^{m} Q_{\tau}(u, v)} \mathrm{d} u \mathrm{~d} v\right) \mathrm{d} \tau,
$$

where

$$
Q_{\tau}(u, v):=\varphi\left(t_{0}(u, v), u, v\right)-\varphi\left(t_{0}\left(u-\tau, v+2^{m+j-k} \tau\right), u-\tau, v+2^{m+j-k} \tau\right) .
$$

To estimate the bilinear operator $T_{j, m, k}(f, g)$ and obtain (5.26), we use the Hörmander theorem on the nondegenerate phase [26]. To this aim, we need to establish Proposition 5.3 below. Let us postpone the proof of Proposition 5.3 for the moment. We now turn to $\left\|T_{j, m, k}(f, g)\right\|_{L^{2}(\mathbb{R})}^{2}$ in (5.27). Let $\tau_{0}:=2^{-\frac{2 m+j-k}{3}}$; noting that $\Phi$ is supported on $\left\{t \in \mathbb{R}: \frac{1}{8} \leq|t| \leq 8\right\}$, we split it as

$$
\begin{aligned}
\left\|T_{j, m, k}(f, g)\right\|_{L^{2}(\mathbb{R})}^{2}= & \phi^{2}\left(\frac{\gamma^{\prime}\left(2^{-j}\right)}{2^{m+j-k}}\right) \int_{|\tau| \leq \tau_{0}}\left(\int_{-\infty}^{\infty} \int_{-\infty}^{\infty} F_{\tau}(u) G_{\tau}(v) e^{i 2^{m} Q_{\tau}(u, v)} \mathrm{d} u \mathrm{~d} v\right) \mathrm{d} \tau \\
& +\phi^{2}\left(\frac{\gamma^{\prime}\left(2^{-j}\right)}{2^{m+j-k}}\right) \int_{\tau_{0}<|\tau| \leq 16}\left(\int_{-\infty}^{\infty} \int_{-\infty}^{\infty} F_{\tau}(u) G_{\tau}(v) e^{i 2^{m} Q_{\tau}(u, v)} \mathrm{d} u \mathrm{~d} v\right) \mathrm{d} \tau .
\end{aligned}
$$

By the Hölder inequality and Plancherel formula, it is easy to see that $\left\|F_{\tau}\right\|_{L^{1}(\mathbb{R})} \leq\|f\|_{L^{2}(\mathbb{R})}^{2}$ and $\left\|G_{\tau}\right\|_{L^{1}(\mathbb{R})} \leq\|g\|_{L^{2}(\mathbb{R})}^{2}$. On the other hand, we have

$$
\left(\int_{\tau_{0}<|\tau| \leq 16}\left\|F_{\tau}\right\|_{L^{2}(\mathbb{R})}^{2} \mathrm{~d} \tau\right)^{\frac{1}{2}}=\left(\int_{\tau_{0}<|\tau| \leq 16} \int_{-\infty}^{\infty}|(\hat{f} \Phi)(x) \overline{(\hat{f} \Phi)(x-\tau)}|^{2} \mathrm{~d} x \mathrm{~d} \tau\right)^{\frac{1}{2}} \leq\|f\|_{L^{2}(\mathbb{R})}^{2},
$$

and $\left(\int_{\tau_{0}<|\tau| \leq 16}\left\|G_{\tau}\right\|_{L^{2}(\mathbb{R})}^{2} \mathrm{~d} \tau\right)^{\frac{1}{2}} \leq 2^{-\frac{m+j-k}{2}}\|g\|_{L^{2}(\mathbb{R})}^{2}$. With all the estimates, from $\underline{5.31}$, by the Hölder inequality and Hörmander [26, Theorem 1.1], we bound $\left\|T_{j, m, k}(f, g)\right\|_{L^{2}(\mathbb{R})}^{2}$ by

$$
\begin{aligned}
& \int_{|\tau| \leq \tau_{0}}\left\|F_{\tau}\right\|_{L^{1}(\mathbb{R})}\left\|G_{\tau}\right\|_{L^{1}(\mathbb{R})} \mathrm{d} \tau+\int_{\tau_{0}<\tau \mid \leq 16}\left(2^{m}\left|\tau_{0}\right|\right)^{-\frac{1}{2}}\left\|F_{\tau}\right\|_{L^{2}(\mathbb{R})}\left\|G_{\tau}\right\|_{L^{2}(\mathbb{R})} \mathrm{d} \tau . \\
\leq & \tau_{0}\|f\|_{L^{2}(\mathbb{R})}^{2}\|g\|_{L^{2}(\mathbb{R})}^{2}+\left(2^{m}\left|\tau_{0}\right|\right)^{-\frac{1}{2}}\left(\int_{\tau_{0}<|\tau| \leq 16}\left\|F_{\tau}\right\|_{L^{2}(\mathbb{R})}^{2} \mathrm{~d} \tau\right)^{\frac{1}{2}}\left(\int_{\tau_{0}<|\tau| \leq 16}\left\|G_{\tau}\right\|_{L^{2}(\mathbb{R})}^{2} \mathrm{~d} \tau\right)^{\frac{1}{2}} \\
\leq & \left(\tau_{0}+\left(2^{m}\left|\tau_{0}\right|\right)^{-\frac{1}{2}} 2^{-\frac{m+j-k}{2}}\right)\|f\|_{L^{2}(\mathbb{R})}^{2}\|g\|_{L^{2}(\mathbb{R})}^{2} \lesssim 2^{-\frac{2 m+j-k}{3}}\|f\|_{L^{2}(\mathbb{R})}^{2}\|g\|_{L^{2}(\mathbb{R})}^{2} .
\end{aligned}
$$

Thus, we obtain (5.26), which leads to 5.16). 
Proposition 5.3. Let $u, v, u-\tau, v+2^{m+j-k} \tau \in \operatorname{supp} \Phi$. Then, there exists a positive constant $C$ such that

$$
\left|\frac{d^{2} Q_{\tau}}{d u d v}(u, v)\right| \geq C|\tau|
$$

where $j$ is large enough.

Proof. Recall that

$$
Q_{\tau}(u, v)=\varphi\left(t_{0}(u, v), u, v\right)-\varphi\left(t_{0}\left(u-\tau, v+2^{m+j-k} \tau\right), u-\tau, v+2^{m+j-k} \tau\right)
$$

and

$$
\varphi(t, u, v)=-u t-v 2^{k-m} \gamma\left(2^{-j} t\right) .
$$

Let $\Psi(u, v):=\varphi\left(t_{0}(u, v), u, v\right)$. Then

$$
\Psi(u, v)=-u t_{0}(u, v)-v 2^{k-m} \gamma\left(2^{-j} t_{0}(u, v)\right),
$$

where $t_{0}(u, v)$ satisfies $\varphi_{1}^{\prime}\left(t_{0}(u, v), u, v\right)=0$. The definition of $t_{0}(u, v)$ leads to

$$
-u-v 2^{-m-j+k} \gamma^{\prime}\left(2^{-j} t_{0}(u, v)\right)=0 .
$$

Furthermore, we have

$$
\frac{\mathrm{d} t_{0}}{\mathrm{~d} u}(u, v)=-\frac{1}{v} \frac{2^{m+2 j-k}}{\gamma^{\prime \prime}\left(2^{-j} t_{0}(u, v)\right)}
$$

and

$$
\frac{\mathrm{d} t_{0}}{\mathrm{~d} v}(u, v)=-\frac{1}{v} \frac{\gamma^{\prime}\left(2^{-j} t_{0}(u, v)\right)}{2^{-j} \gamma^{\prime \prime}\left(2^{-j} t_{0}(u, v)\right)}=\frac{u}{v^{2}} \frac{2^{m+2 j-k}}{\gamma^{\prime \prime}\left(2^{-j} t_{0}(u, v)\right)}
$$

Therefore,

$$
\frac{\mathrm{d} \Psi}{\mathrm{d} v}(u, v)=-2^{k-m} \gamma\left(2^{-j} t_{0}(u, v)\right)
$$

and from 5.35),

$$
\frac{\mathrm{d}^{2} \Psi}{\mathrm{d} u \mathrm{~d} v}(u, v)=\frac{u}{v} \frac{\mathrm{d} t_{0}}{\mathrm{~d} u}(u, v)
$$

Then

$$
\frac{\mathrm{d}^{3} \Psi}{\mathrm{d}^{2} u \mathrm{~d} v}(u, v)=\frac{1}{v^{2}} \frac{2^{m+2 j-k}}{\gamma^{\prime \prime}\left(2^{-j} t_{0}(u, v)\right)}\left(\frac{\gamma^{\prime} \gamma^{\prime \prime \prime}-\left(\gamma^{\prime \prime}\right)^{2}}{\left(\gamma^{\prime \prime}\right)^{2}}\right)\left(2^{-j} t_{0}(u, v)\right) .
$$

From (1.1), we have

$$
\left|\left(\frac{\gamma^{\prime} \gamma^{\prime \prime \prime}-\left(\gamma^{\prime \prime}\right)^{2}}{\left(\gamma^{\prime \prime}\right)^{2}}\right)\left(2^{-j} t_{0}(u, v)\right)\right| \approx 1 .
$$


From (2.3) and (2.1), note that $\gamma$ is either odd or even, $\gamma^{\prime}$ is increasing on $(0, \infty)$ and $\frac{\gamma^{\prime}\left(2^{-j}\right)}{2^{m+j-k}} \in$ supp $\phi$; thus, we have

$$
\left|\frac{2^{m+2 j-k}}{\gamma^{\prime \prime}\left(2^{-j} t_{0}(u, v)\right)}\right|=\left|\frac{2^{m+j-k}}{\gamma^{\prime}\left(2^{-j}\right)} \frac{\gamma^{\prime}\left(2^{-j}\right) t_{0}(u, v)}{\gamma^{\prime}\left(2^{-j} t_{0}(u, v)\right)} \frac{\gamma^{\prime}\left(2^{-j} t_{0}(u, v)\right)}{2^{-j} t_{0}(u, v) \gamma^{\prime \prime}\left(2^{-j} t_{0}(u, v)\right)}\right| \approx 1 .
$$

Since $v \in \operatorname{supp} \Psi$, from (5.41) and (5.42),

$$
\left|\frac{\mathrm{d}^{3} \Psi}{\mathrm{d}^{2} u \mathrm{~d} v}(u, v)\right| \approx 1 .
$$

On the other hand, we write $\frac{\mathrm{d}^{3} \Psi}{\mathrm{d} v \mathrm{~d} u \mathrm{~d} v}(u, v)$ as

$$
\frac{1}{v^{2}} \frac{\gamma^{\prime}\left(2^{-j} t_{0}(u, v)\right)}{2^{-j} \gamma^{\prime \prime}\left(2^{-j} t_{0}(u, v)\right)}\left(\frac{\gamma^{\prime} \gamma^{\prime \prime \prime}-\left(\gamma^{\prime \prime}\right)^{2}}{\left(\gamma^{\prime \prime}\right)^{2}}\right)\left(2^{-j} t_{0}(u, v)\right)-\frac{1}{v^{2}} \frac{\gamma^{\prime}\left(2^{-j} t_{0}(u, v)\right)}{2^{-j} \gamma^{\prime \prime}\left(2^{-j} t_{0}(u, v)\right)} \text {. }
$$

As in (5.43), we have

$$
\left|\frac{\mathrm{d}^{3} \Psi}{\mathrm{d} v \mathrm{~d} u \mathrm{~d} v}(u, v)\right| \lesssim 1 .
$$

By the mean value theorem, we rewrite $\frac{\mathrm{d}^{2} Q_{\tau}}{\mathrm{d} u \mathrm{~d} v}(u, v)$ as

$$
\frac{\mathrm{d}^{3} \Psi}{\mathrm{d}^{2} u \mathrm{~d} v}\left(u-\theta_{1} \tau, v\right) \cdot \tau-\frac{\mathrm{d}^{3} \Psi}{\mathrm{d} \nu \mathrm{d} u \mathrm{~d} \nu}\left(u-\tau, v+\theta_{2} 2^{m+j-k} \tau\right) \cdot 2^{m+j-k} \tau,
$$

where $\theta_{1}, \theta_{2} \in[0,1]$. From (5.43), (5.45), and $2^{m+j-k} \approx \gamma^{\prime}\left(2^{-j}\right)$ when $j$ is large enough, we have

$$
\left|\frac{\mathrm{d}^{2} Q_{\tau}}{\mathrm{d} u \mathrm{~d} v}(u, v)\right| \gtrsim\left(1-2^{m+j-k}\right)|\tau| \gtrsim|\tau| \text {. }
$$

This finishes the proof of Proposition 5.3 .

\subsection{Another estimate by $\sigma$-uniformity and the $T T^{*}$ argument}

In this subsection, we set up

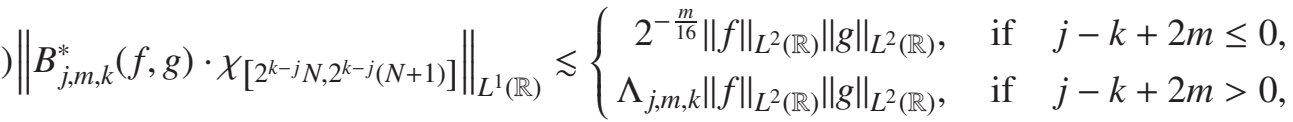

where $\Lambda_{j, m, k}:=\max \left\{2^{\frac{15 m}{16}+\frac{j}{2}-\frac{k}{2}}, 2^{\frac{m}{16}}\left(\max \left\{2^{m+j-k}, 2^{-\frac{m}{4}}\right\}\right)^{\frac{1}{2}}\right\}$.

We start by quoting a lemma stated in [16, Lemma 4.4] or [24, Lemma 3.3], which is a slight variant of [34, Theorem 7.1] and is called the $\sigma$-uniformity argument. Indeed, this argument can be traced back to Christ et al. [8] and Gowers [21]. Let $\sigma \in(0,1], \mathbb{I} \subset \mathbb{R}$ be a fixed bounded interval, and $U(\mathbb{I})$ be a nontrivial subset of $L^{2}(\mathbb{I})$ with $\|u\|_{L^{2}(\mathbb{I})} \leq C$ uniformly for every element of $u \in U(\mathbb{I})$. We say that a function $f \in L^{2}(\mathbb{I})$ is $\sigma$-uniform in $U(\mathbb{I})$ if

$$
\left|\int_{\mathbb{I}} f(x) \overline{u(x)} \mathrm{d} x\right| \leq \sigma\|f\|_{L^{2}(\mathbb{I})}
$$

for all $u \in U(\mathbb{I})$. 
Lemma 5.4. [34 Theorem 7.1] Let $\mathcal{L}$ be a bounded sublinear functional from $L^{2}(\mathbb{I})$ to $\mathbb{C}, S_{\sigma}$ be the set of all functions that are $\sigma$-uniform in $U(\mathbb{I})$,

$$
\mathcal{A}_{\sigma}:=\sup _{f \in S_{\sigma}} \frac{|\mathcal{L}(f)|}{\|f\|_{L^{2}(\mathbb{I})}} \quad \text { and } \quad \mathcal{M}:=\sup _{u \in U(\mathbb{I})}|\mathcal{L}(u)| .
$$

Then, for all $f \in L^{2}(\mathbb{I})$, we have

$$
|\mathcal{L}(f)| \leq \max \left\{\mathcal{A}_{\sigma}, 2 \sigma^{-1} \mathcal{M}\right\}\|f\|_{L^{2}(\mathbb{I})} .
$$

We now turn to the proof of 5.47) by using Lemma 5.4. Let $\mathbb{I}:=\operatorname{supp} \Phi$, and for any given $g \in L^{2}(\mathbb{R})$, let

$$
\mathcal{L}\left(\chi_{\mathbb{I}} \hat{f}\right):=\left\|B_{j, m, k}^{*}(f, g) \cdot \chi_{\left[2^{k-j} N, 2^{k-j}(N+1)\right]}\right\|_{L^{1}(\mathbb{R})} .
$$

\section{Step 1: Estimates for $\mathcal{A}_{\sigma}$}

We split the interval $\left[2^{k-j} N, 2^{k-j}(N+1)\right]$ as $\bigcup_{w=1}^{2^{m}} I_{w}$, where

$$
I_{w}:=\left[a_{w}, a_{w+1}\right]:=\left[2^{k-j} N+\frac{w-1}{2^{m+j-k}}, 2^{k-j} N+\frac{w}{2^{m+j-k}}\right]
$$

Furthermore, let us set

$$
I_{w}^{\prime}:=\left[2^{k-j} N+\frac{w-1}{2^{m+j-k}}-2^{2+\frac{2}{C_{1}}} 2^{m}, 2^{k-j} N+\frac{w}{2^{m+j-k}}+2^{2+\frac{2}{C_{1}}} 2^{m}\right] .
$$

It is easy to see that $x-2^{k} \gamma\left(2^{-j} t\right) \in I_{w}^{\prime}$ if $x \in I_{w}$, since $\frac{\gamma^{\prime}(2 t)}{\gamma^{\prime}(t)} \leq 2^{\frac{2}{C_{1}}}$ for all $t \in$ supp $\rho$. We write $B_{j, m, k}^{*}(f, g)(x) \cdot \chi_{\left[2^{k-j} N, 2^{k-j}(N+1)\right]}(x)$ as

$$
2^{\frac{m+j-k}{2}} \phi\left(\frac{\gamma^{\prime}\left(2^{-j}\right)}{2^{m+j-k}}\right) \sum_{w=1}^{2^{m}} \chi_{I_{w}}(x) \int_{-\infty}^{\infty} \int_{-\infty}^{\infty} \hat{f}(\xi) \Phi(\xi) \widehat{\dot{g}_{w}}(\eta) e^{i 2^{m+j-k} \xi x} e^{i \eta x} I_{j, m, k}(\xi, \eta) \mathrm{d} \xi \mathrm{d} \eta
$$

where $I_{j, m, k}$ can be found in (5.19) and $\dot{g}_{w}:=\left(\chi_{I_{w}^{\prime}} \cdot \breve{\Phi} * g\right)$.

For $h \in L^{2}(\mathbb{R})$, based on $\xi \in \operatorname{supp} \Phi$ and $2^{m+j-k}\left|x-a_{w}\right| \leq 1$ for all $x \in I_{w}$, by Taylor's theorem $e^{i 2^{m+j-k} \xi\left(x-a_{w}\right)}$, it is safe to split $\int_{-\infty}^{\infty} B_{j, m, k}^{*}(f, g)(x) \cdot \chi_{\left[2^{k-j} N, 2^{k-j}(N+1)\right]}(x) h(x) \mathrm{d} x$ as the sum of $I$ and $I I$, where

$$
\left\{\begin{aligned}
I:= & 2^{\frac{m+j-k}{2}} \phi\left(\frac{\gamma^{\prime}\left(2^{-j}\right)}{2^{m+j-k}}\right) \sum_{w=1}^{2^{m}} \sum_{l \in \mathbb{N}} \frac{i^{l}}{l !} \int_{-\infty}^{\infty} \int_{-\infty}^{\infty} \hat{f}(\xi) \Phi(\xi) e^{i 2^{m+j-k} \xi a_{w}} \widehat{\dot{g}_{w}}(\eta)(1-\mathcal{G}(\eta)) I_{j, m, k}(\xi, \eta) \xi^{l} \\
& \times \mathcal{F}^{-1}\left(\left(2^{m+j-k}\left(\cdot-a_{w}\right)\right)^{l} \chi_{I_{w}}(\cdot) h(\cdot)\right)(\eta) \mathrm{d} \xi \mathrm{d} \eta \\
I I:= & 2^{\frac{m+j-k}{2}} \phi\left(\frac{\gamma^{\prime}\left(2^{-j}\right)}{2^{m+j-k}}\right) \sum_{w=1}^{2^{m}} \sum_{l \in \mathbb{N}} \frac{i^{l}}{l !} \int_{-\infty}^{\infty} \int_{-\infty}^{\infty} \hat{f}(\xi) \Phi(\xi) e^{i 2^{m+j-k} \xi a_{w}} \widehat{\hat{g}_{w}}(\eta) \mathcal{G}(\eta) I_{j, m, k}(\xi, \eta) \xi^{l} \\
& \times \mathcal{F}^{-1}\left(\left(2^{m+j-k}\left(\cdot-a_{w}\right)\right)^{l} \chi_{I_{w}}(\cdot) h(\cdot)\right)(\eta) \mathrm{d} \xi \mathrm{d} \eta
\end{aligned}\right.
$$

$\mathcal{G}$ is a bump function supported on $\left\{t \in \mathbb{R}: 2^{-6-\frac{2}{C_{1}}} \leq|t| \leq 2^{6+\frac{2}{C_{1}}}\right\}$ such that $\mathcal{G}(t)=1$ on $\left\{t \in \mathbb{R}: 2^{-5-\frac{2}{C_{1}}} \leq|t| \leq 2^{5+\frac{2}{C_{1}}}\right\}$, and $\mathcal{F}^{-1}(f)$ means the inverse Fourier transform of $f$. 
For $I$. First, we obtain that $\left|I_{j, m, k}\right| \leq 2^{-m}$. Indeed, note that $|\eta| \leq 2^{-5-\frac{2}{C_{1}}}$ or $|\eta| \geq 2^{5+\frac{2}{C_{1}}}$, and $\xi \in \operatorname{supp} \Phi, \frac{\gamma^{\prime}\left(2^{-j}\right)}{2^{m+j-k}} \in \operatorname{supp} \phi$; we have $\left|\varphi_{1}^{\prime}(t, \xi, \eta)\right| \geq|\xi|-\left|2^{-m-j+k} \gamma^{\prime}\left(2^{-j} t\right) \eta\right| \geq \frac{1}{16}$ or $\left|\varphi_{1}^{\prime}(t, \xi, \eta)\right| \geq$ $\left|2^{-m-j+k} \gamma^{\prime}\left(2^{-j} t\right) \eta\right|-|\xi| \geq 8$. Note that $\varphi_{1}^{\prime \prime}(t, \xi, \eta)=-2^{-m-j+k} 2^{-j} \gamma^{\prime \prime}\left(2^{-j} t\right) \eta$, by the Van der Corput lemma, for example, see [42, P. 332, Proposition 2], we have $\left|I_{j, m}\right| \leq 2^{-m}$. Therefore, by the Hölder inequality, Plancherel formula and Cauchy-Schwarz inequality, we bound $|I|$ by

$$
\begin{aligned}
& 2^{\frac{m+j-k}{2}} \phi\left(\frac{\gamma^{\prime}\left(2^{-j}\right)}{2^{m+j-k}}\right) \sum_{w=1}^{2^{m}} \sum_{l \in \mathbb{N}} \frac{8^{l}}{l !} 2^{-m}\left\|\chi_{\mathbb{I}} \hat{f}\right\|_{L^{2}(\mathbb{R})}\left\|\dot{g}_{w}\right\|_{L^{2}(\mathbb{R})}\left\|\left(2^{m+j-k}\left(\cdot-a_{w}\right)\right)^{l} \chi_{I_{w}}(\cdot) h(\cdot)\right\|_{L^{2}(\mathbb{R})} \\
\leq & 2^{\frac{m+j-k}{2}} \phi\left(\frac{\gamma^{\prime}\left(2^{-j}\right)}{2^{m+j-k}}\right) \sum_{l \in \mathbb{N}} \frac{8^{l}}{l !} 2^{-m}\left\|\chi_{\mathbb{I}} \hat{f}\right\|_{L^{2}(\mathbb{R})}\left[\sum_{w=1}^{2^{m}}\left\|\dot{g}_{w}\right\|_{L^{2}(\mathbb{R})}^{2}\right]^{\frac{1}{2}}\left[\sum_{w=1}^{2^{m}}\left\|\left(2^{m+j-k}\left(\cdot-a_{w}\right)\right)^{l} \chi_{I_{w}}(\cdot) h(\cdot)\right\|_{L^{2}(\mathbb{R})}^{2}\right]^{\frac{1}{2}} \\
\leq & 2^{\frac{-m+j-k}{2}}\left\|\chi_{\mathbb{I}} \hat{f}\right\|_{L^{2}(\mathbb{R})}\left[\sum_{w=1}^{2^{m}}\left\|\dot{g}_{w}\right\|_{L^{2}(\mathbb{R})}^{2}\right]^{\frac{1}{2}}\|h\|_{L^{2}(\mathbb{R})},
\end{aligned}
$$

where the last inequality is a result of the fact that $2^{m+j-k}\left|x-a_{w}\right| \leq 1$ for all $x \in I_{w}$. Based on the overlap property of $\left\{I_{w}^{\prime}\right\}_{w=1}^{2^{m}}$, we have

$$
\begin{cases}{\left[\sum_{w=1}^{2^{m}}\left\|\dot{g}_{w}\right\|_{L^{2}(\mathbb{R})}^{2}\right]^{\frac{1}{2}} \lesssim\|g\|_{L^{2}(\mathbb{R})},} & \text { if } \quad j-k+2 m \leq 0 ; \\ {\left[\sum_{w=1}^{2^{m}}\left\|\dot{g}_{w}\right\|_{L^{2}(\mathbb{R})}^{2}\right]^{\frac{1}{2}} \lesssim 2^{\frac{2 m+j-k}{2}}\|g\|_{L^{2}(\mathbb{R})},} & \text { if } \quad j-k+2 m>0 .\end{cases}
$$

Therefore,

$$
|I| \lesssim\left\{\begin{array}{ccc}
2^{-\frac{m}{2}}\left\|\chi_{\mathbb{I}} \hat{f}\right\|_{L^{2}(\mathbb{R})}\|g\|_{L^{2}(\mathbb{R})} 2^{\frac{j-k}{2}}\|h\|_{L^{2}(\mathbb{R})}, & \text { if } & j-k+2 m \leq 0, \\
2^{\frac{m+j-k}{2}}\left\|\chi_{\mathbb{I}} \hat{f}\right\|_{L^{2}(\mathbb{R})}\|g\|_{L^{2}(\mathbb{R})} 2^{\frac{j-k}{2}}\|h\|_{L^{2}(\mathbb{R})}, & \text { if } & j-k+2 m>0 .
\end{array}\right.
$$

For $I I$, we write $I I$ as $I I_{1}+I I_{2}$ by using the decomposition in (5.23). As in $I$, it is easy to see that

$$
\left|I I_{1}\right| \lesssim\left\{\begin{array}{lll}
2^{-m}\left\|\chi_{\mathbb{I}} \hat{f}\right\|_{L^{2}(\mathbb{R})}\|g\|_{L^{2}(\mathbb{R})} 2^{\frac{j-k}{2}}\|h\|_{L^{2}(\mathbb{R})}, & \text { if } & j-k+2 m \leq 0, \\
2^{\frac{j-k}{2}}\left\|\chi_{\mathbb{I}} \hat{f}\right\|_{L^{2}(\mathbb{R})}\|g\|_{L^{2}(\mathbb{R})} 2^{\frac{j-k}{2}}\|h\|_{L^{2}(\mathbb{R})}, & \text { if } \quad j-k+2 m>0 .
\end{array}\right.
$$

$I I_{2}$ can be written as

$$
\begin{aligned}
& 2^{\frac{m+j-k}{2}} \phi\left(\frac{\gamma^{\prime}\left(2^{-j}\right)}{2^{m+j-k}}\right) \sum_{w=1}^{2^{m}} \sum_{l \in \mathbb{N}} \frac{i^{l}}{l !} \int_{-\infty}^{\infty} \int_{-\infty}^{\infty} \hat{f}(\xi) \Phi(\xi) e^{i 2^{m+j-k} \xi a_{w}} \widehat{\dot{g}_{w}}(\eta) \mathcal{G}(\eta) e^{i 2^{m} \varphi\left(t_{0}(\xi, \eta), \xi, \eta\right)} \\
& \times\left(\frac{2 \pi}{-i 2^{m} \varphi_{1}^{\prime \prime}\left(t_{0}(\xi, \eta), \xi, \eta\right)}\right)^{\frac{1}{2}} \rho\left(t_{0}(\xi, \eta)\right) \xi^{l} \mathcal{F}^{-1}\left(\left(2^{m+j-k}\left(\cdot-a_{w}\right)\right)^{l} \chi_{I_{w}}(\cdot) h(\cdot)\right)(\eta) \mathrm{d} \xi \mathrm{d} \eta \\
= & :\left(\frac{2 \pi}{-i}\right)^{\frac{1}{2}} 2^{\frac{j-k}{2}} \phi\left(\frac{\gamma^{\prime}\left(2^{-j}\right)}{2^{m+j-k}}\right) \sum_{w=1}^{2^{m}} \sum_{l \in \mathbb{N}} \frac{i^{l}}{l !} \int_{-\infty}^{\infty} \Upsilon(\eta) \widehat{\dot{g}_{w}}(\eta) \mathcal{F}^{-1}\left(\left(2^{m+j-k}\left(\cdot-a_{w}\right)\right)^{l} \chi_{I_{w}}(\cdot) h(\cdot)\right)(\eta) \mathrm{d} \eta,
\end{aligned}
$$


where

$$
\left\{\begin{array}{l}
\Upsilon(\eta):=\int_{\mathbb{I}} v(\xi, \eta) \hat{f}(\xi) e^{i 2^{m} \varphi\left(t_{0}(\xi, \eta), \xi, \eta\right)} e^{i 2^{m+j-k} \xi a_{w}} \mathrm{~d} \xi \\
v(\xi, \eta):=\rho\left(t_{0}(\xi, \eta)\right)\left(\frac{1}{\varphi_{1}^{\prime \prime}\left(t_{0}(\xi, \eta), \xi, \eta\right)}\right)^{\frac{1}{2}} \mathcal{G}(\eta) \xi^{l} \Phi(\xi)
\end{array}\right.
$$

To apply Lemma 5.4, we first define

$$
U(\mathbb{I}):=\left\{u_{\eta, r}(\xi) \in L^{2}(\mathbb{I}): r \in \mathbb{R}, 2^{-6-\frac{2}{C_{1}}} \leq|\eta| \leq 2^{6+\frac{2}{C_{1}}}\right\},
$$

where $u_{\eta, r}(\xi):=v(\xi, \eta) e^{-i 2^{m} \varphi\left(t_{0}(\xi, \eta), \xi, \eta\right)} e^{-i r \xi}$. From 2.11), 5.21) and $\frac{\gamma^{\prime}\left(2^{-j}\right)}{2^{m+j-k}} \in \operatorname{supp} \phi$, it is easy to see that $\left\|u_{\eta, r}\right\|_{L^{2}(\mathbb{I})} \leq C$ uniformly for every element of $u_{\eta, r} \in U(\mathbb{I})$. To estimate $\mathcal{A}_{\sigma}$, we first assume that $\chi_{\mathbb{I}} \hat{f} \in L^{2}(\mathbb{I})$ is $\sigma$-uniform in $U(\mathbb{I})$, which further implies that

$$
|\Upsilon(\eta)| \leq \sigma\left\|\chi_{\mathbb{I}} \hat{f}\right\|_{L^{2}(\mathbb{R})} .
$$

Regarding $I$, let $\sigma:=2^{-\frac{m}{16}}$; it is easy to see that

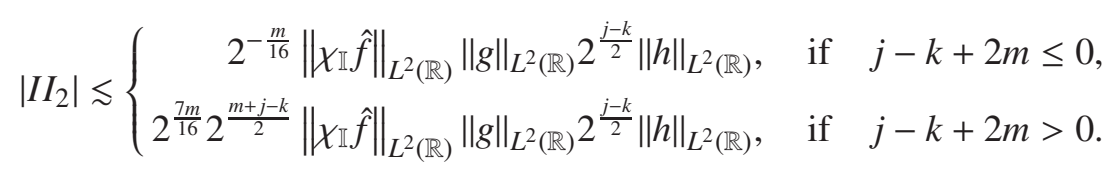

From (5.48), (5.49), and (5.52), $\left|\int_{-\infty}^{\infty} B_{j, m, k}^{*}(f, g)(x) \cdot \chi_{\left[2^{k-j} N, 2^{k-j}(N+1)\right]}(x) h(x) \mathrm{d} x\right|$ can be bounded by

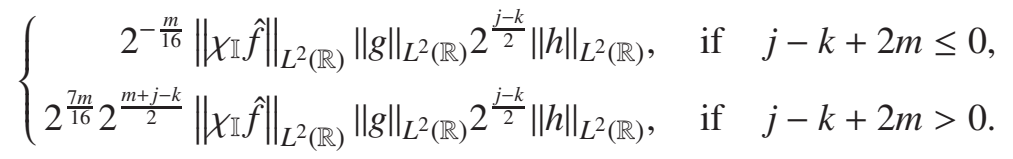

Let $h:=\operatorname{sgn}\left(B_{j, m, k}^{*}(f, g)\right) \cdot \chi_{\left[2^{k-j} N, 2^{k-j}(N+1)\right]} \cdot\left\|B_{j, m, k}^{*}(f, g) \cdot \chi_{\left[2^{k-j} N, 2^{k-j}(N+1)\right]}\right\|_{L^{1}(\mathbb{R})}$ can be bounded by

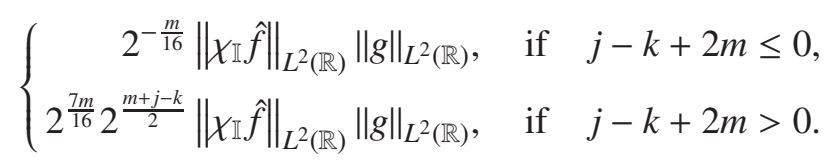

It is easy to see that by the definition of $\mathcal{L}\left(\chi_{\mathbb{I}} \hat{f}\right)$ that

$$
\mathcal{A}_{\sigma} \lesssim\left\{\begin{aligned}
2^{-\frac{m}{16}}\|g\|_{L^{2}(\mathbb{R})}, & \text { if } \quad j-k+2 m \leq 0, \\
2^{\frac{7 m}{16}} 2^{\frac{m+j-k}{2}}\|g\|_{L^{2}(\mathbb{R})}, & \text { if } \quad j-k+2 m>0 .
\end{aligned}\right.
$$

\section{Step 2: Estimates for $\mathcal{M}$}

For $\varpi \in L^{\infty}(\mathbb{R})$, let $x:=2^{k-j} x+2^{k} \gamma\left(2^{-j} t\right) ; \int_{-\infty}^{\infty} B_{j, m, k}^{*}(f, g)(x) \varpi(x) \mathrm{d} x$ can be written as

$$
\begin{aligned}
2^{\frac{m-j+k}{2}} \phi\left(\frac{\gamma^{\prime}\left(2^{-j}\right)}{2^{m+j-k}}\right) \int_{-\infty}^{\infty} \int_{-\infty}^{\infty} \check{\Phi} * f\left(2^{m} x+2^{m+j} \gamma\left(2^{-j} t\right)-2^{m} t\right) \\
\quad \times \check{\Phi} * g\left(2^{k-j} x\right) \varpi\left(2^{k-j} x+2^{k} \gamma\left(2^{-j} t\right)\right) \rho(t) \mathrm{d} t \mathrm{~d} x .
\end{aligned}
$$


Therefore,

$$
\left|\int_{-\infty}^{\infty} B_{j, m, k}^{*}(f, g)(x) \varpi(x) \mathrm{d} x\right| \leq\|g\|_{L^{2}(\mathbb{R})}\|\mathcal{T}(\varpi)\|_{L^{2}(\mathbb{R})},
$$

where $\mathcal{T}(\varpi)(x)$ is defined as

$$
2^{\frac{m}{2}} \phi\left(\frac{\gamma^{\prime}\left(2^{-j}\right)}{2^{m+j-k}}\right) \int_{-\infty}^{\infty} \check{\Phi} * f\left(2^{m} x+2^{m+j} \gamma\left(2^{-j} t\right)-2^{m} t\right) \varpi\left(2^{k-j} x+2^{k} \gamma\left(2^{-j} t\right)\right) \rho(t) \mathrm{d} t .
$$

Let $\chi_{\mathbb{I}}(\xi) \hat{f}(\xi):=v(\xi, \eta) e^{-i 2^{m} \varphi\left(t_{0}(\xi, \eta), \xi, \eta\right)} e^{-i r \xi}$ and $x:=x+2^{-m} r,\|\mathcal{T}(\varpi)\|_{L^{2}(\mathbb{R})}^{2}$ equals to

$$
2^{m} \phi^{2}\left(\frac{\gamma^{\prime}\left(2^{-j}\right)}{2^{m+j-k}}\right) \int_{-\infty}^{\infty}\left|\int_{-\infty}^{\infty} \kappa(x, t) \varpi\left(2^{k-j} x+2^{k-j-m} r+2^{k} \gamma\left(2^{-j} t\right)\right) \rho(t) \mathrm{d} t\right|^{2} \mathrm{~d} x,
$$

where

$$
\kappa(x, t):=\int_{-\infty}^{\infty} \Phi(\xi) v(\xi, \eta) e^{i 2^{m}\left(x \xi+2^{j} \gamma\left(2^{-j} t\right) \xi-t \xi-\varphi\left(t_{0}(\xi, \eta), \xi, \eta\right)\right)} \mathrm{d} \xi
$$

Furthermore, let us set

$$
K(\xi, x, t):=x \xi+2^{j} \gamma\left(2^{-j} t\right) \xi-t \xi-\varphi\left(t_{0}(\xi, \eta), \xi, \eta\right)
$$

we have $K_{1}^{\prime}(\xi, x, t):=x+2^{j} \gamma\left(2^{-j} t\right)-t+t_{0}(\xi, \eta)$. There exists a positive constant $\delta$ such that $\left|2^{j} \gamma\left(2^{-j} t\right)-t+t_{0}(\xi, \eta)\right| \leq \frac{\delta}{4}$ for $j$ large enough. Therefore, we can split (5.56) as

$$
\|\mathcal{T}(\varpi)\|_{L^{2}(\mathbb{R})}^{2}=:\|\mathcal{T}(\varpi), a\|_{L^{2}(\mathbb{R})}^{2}+\|\mathcal{T}(\varpi), b\|_{L^{2}(\mathbb{R})}^{2}
$$

by $1=(1-\Delta(x))+\Delta(x)$ on the right-hand side of (5.56), where $\Delta$ is a bump function supported on $\{x \in \mathbb{R}:|x| \leq \delta\}$ such that $\Delta(x)=1$ on $\left\{x \in \mathbb{R}:|x| \leq \frac{\delta}{2}\right\}$. For $\|\mathcal{T}(\varpi), a\|_{L^{2}(\mathbb{R})}^{2}$, we have $\left|K_{1}^{\prime}(\xi, x, t)\right| \geq|x|-\left|2^{j} \gamma\left(2^{-j} t\right)-t+t_{0}(\xi, \eta)\right| \geq|x|-\frac{\delta}{4} \geq \frac{|x|}{2}$. Note that $K_{1}^{\prime \prime}(\xi, x, t)=-\frac{1}{\eta} \frac{2^{m+2 j-k}}{\gamma^{\prime \prime}\left(2^{-j} t_{0}(\xi, \eta)\right)}$, by the Van der Corput lemma, for example, see [42, P. 332, Proposition 2], we have that $|\kappa(x, t)| \leq$ $2^{-m}|x|^{-1}$. Then

$$
\|\mathcal{T}(\varpi), a\|_{L^{2}(\mathbb{R})}^{2} \lesssim 2^{m}\left[\int_{|x| \geq \frac{\delta}{2}}\left(2^{-m}|x|^{-1}\right)^{2} \mathrm{~d} x\right] \cdot\|\varpi\|_{L^{\infty}(\mathbb{R})}^{2} \lesssim 2^{-m}\|\varpi\|_{L^{\infty}(\mathbb{R})}^{2} .
$$

We now turn to $\|\mathcal{T}(\varpi), b\|_{L^{2}(\mathbb{R})}^{2}$; it has been defined as

$$
2^{m} \phi^{2}\left(\frac{\gamma^{\prime}\left(2^{-j}\right)}{2^{m+j-k}}\right) \int_{-\infty}^{\infty}\left|\int_{-\infty}^{\infty} \kappa(x, t) \varpi\left(2^{k-j} x+2^{k-j-m} r+2^{k} \gamma\left(2^{-j} t\right)\right) \rho(t) \mathrm{d} t\right|^{2} \Delta(x) \mathrm{d} x .
$$

For $\kappa(x, t)$, whose phase function is $2^{m} K(\xi, x, t)$. Let $\xi(x, t)$ satisfy $K_{1}^{\prime}(\xi(x, t), x, t)=0$; in other words,

$$
x+2^{j} \gamma\left(2^{-j} t\right)-t+t_{0}(\xi(x, t), \eta)=0
$$


By the stationary phase method, we assert that

$$
\kappa(x, t)=e^{i 2^{m} K(\xi(x, t), x, t)}\left(\frac{2 \pi}{-i 2^{m} K_{1}^{\prime \prime}(\xi(x, t), x, t)}\right)^{\frac{1}{2}} \Phi(\xi(x, t)) \cdot v(\xi(x, t), \eta)+O\left(2^{-\frac{3}{2} m}\right) .
$$

From (5.62), we split $\|\mathcal{T}(\varpi), b\|_{L^{2}(\mathbb{R})}^{2}$ as

$$
\|\mathcal{T}(\varpi), b\|_{L^{2}(\mathbb{R})}^{2}=:\|\mathcal{T}(\varpi), b, I\|_{L^{2}(\mathbb{R})}^{2}+\|\mathcal{T}(\varpi), b, I I\|_{L^{2}(\mathbb{R})}^{2} .
$$

Furthermore, it is easy to see that

$$
\|\mathcal{T}(\varpi), b, I I\|_{L^{2}(\mathbb{R})}^{2} \lesssim 2^{-2 m}\|\varpi\|_{L^{\infty}(\mathbb{R})}^{2} .
$$

For $\|\mathcal{T}(\varpi), b, I\|_{L^{2}(\mathbb{R})}^{2}$, which can be written as

$$
\begin{aligned}
& \phi^{2}\left(\frac{\gamma^{\prime}\left(2^{-j}\right)}{2^{m+j-k}}\right) \int_{-\infty}^{\infty} \mid \int_{-\infty}^{\infty} e^{i 2^{m} K(\xi(x, t), x, t)}\left(\frac{1}{K_{1}^{\prime \prime}(\xi(x, t), x, t)}\right)^{\frac{1}{2}} \Phi(\xi(x, t)) \\
& \times\left. v(\xi(x, t), \eta) \varpi\left(2^{k-j} x+2^{k-j-m} r+2^{k} \gamma\left(2^{-j} t\right)\right) \rho(t) \mathrm{d} t\right|^{2} \Delta(x) \mathrm{d} x=:\|T(\varpi)\|_{L^{2}(\mathbb{R})}^{2},
\end{aligned}
$$

where $T(\varpi)(x)$ is defined as

$$
\begin{aligned}
& \phi\left(\frac{\gamma^{\prime}\left(2^{-j}\right)}{2^{m+j-k}}\right) \int_{-\infty}^{\infty} \sqrt{\Delta(x)} e^{i 2^{m} K(\xi(x, t), x, t)}\left(\frac{1}{K_{1}^{\prime \prime}(\xi(x, t), x, t)}\right)^{\frac{1}{2}} \Phi(\xi(x, t)) \cdot v(\xi(x, t), \eta) \\
& \times \varpi\left(2^{k-j} x+2^{k-j-m} r+2^{k} \gamma\left(2^{-j} t\right)\right) \rho(t) \mathrm{d} t .
\end{aligned}
$$

Let

$$
y(x, t):=x+2^{j} \gamma\left(2^{-j} t\right)-t
$$

Note that from (5.20), 5.61), and (5.57), we have $K(\xi(x, t), x, t)=2^{k-m} \eta \gamma\left(-2^{-j} y(x, t)\right)$. Let $Q_{j}(t):=\frac{2^{j} \gamma\left(2^{-j} t\right)}{\gamma^{\prime}\left(2^{-j}\right)}$ and $u:=Q_{j}(t)$. We rewrite $T(\varpi)(x)$ as

$$
\begin{aligned}
& \phi\left(\frac{\gamma^{\prime}\left(2^{-j}\right)}{2^{m+j-k}}\right) \int_{-\infty}^{\infty} \sqrt{\Delta(x)} e^{i 2^{m} 2^{k-m-j} \gamma^{\prime}\left(2^{-j}\right) \eta Q_{j}\left(-y\left(x, Q_{j}^{-1}(u)\right)\right)}\left(\frac{1}{K_{1}^{\prime \prime}\left(\xi\left(x, Q_{j}^{-1}(u)\right), x, Q_{j}^{-1}(u)\right)}\right)^{\frac{1}{2}} \\
& \times \Phi\left(\xi\left(x, Q_{j}^{-1}(u)\right)\right) \cdot v\left(\xi\left(x, Q_{j}^{-1}(u)\right), \eta\right) \varpi\left(2^{k-j} x+2^{k-m-j} r+2^{m} 2^{k-m-j} \gamma^{\prime}\left(2^{-j}\right) u\right) \frac{\rho\left(Q_{j}^{-1}(u)\right)}{\left(Q_{j}\right)^{\prime}\left(Q_{j}^{-1}(u)\right)} \mathrm{d} u .
\end{aligned}
$$

Furthermore, denote $\mathcal{K}(x, u)$ as

$$
\frac{\phi\left(\frac{\gamma^{\prime}\left(2^{-j}\right)}{2^{m+j-k}}\right) \sqrt{\Delta(x)}}{\left[K_{1}^{\prime \prime}\left(\xi\left(x, Q_{j}^{-1}(u)\right), x, Q_{j}^{-1}(u)\right)\right]^{\frac{1}{2}}} \Phi\left(\xi\left(x, Q_{j}^{-1}(u)\right)\right) \cdot v\left(\xi\left(x, Q_{j}^{-1}(u)\right), \eta\right) \frac{\rho\left(Q_{j}^{-1}(u)\right)}{\left(Q_{j}\right)^{\prime}\left(Q_{j}^{-1}(u)\right)},
$$


$T(\varpi)(x)$ can be written as

$$
\int_{-\infty}^{\infty} \mathcal{K}(x, u) e^{i 2^{m} 2^{k-m-j} \gamma^{\prime}\left(2^{-j}\right) \eta Q_{j}\left(-y\left(x, Q_{j}^{-1}(u)\right)\right)} \varpi\left(2^{k-j} x+2^{k-m-j} r+2^{m} 2^{k-m-j} \gamma^{\prime}\left(2^{-j}\right) u\right) \mathrm{d} u .
$$

By the $T T^{*}$ argument, $\|T(\varpi)\|_{L^{2}(\mathbb{R})}^{2}$ is equal to

$$
\begin{aligned}
& \int_{-\infty}^{\infty}\left[\int_{-\infty}^{\infty} \int_{-\infty}^{\infty} \mathcal{K}\left(x, u_{1}\right) \overline{\mathcal{K}\left(x, u_{2}\right)}\right. \\
& \times \varpi\left(2^{k-j} x+2^{k-m-j} r+2^{m} 2^{k-m-j} \gamma^{\prime}\left(2^{-j}\right) u_{1}\right) \overline{\varpi\left(2^{k-j} x+2^{k-m-j} r+2^{m} 2^{k-m-j} \gamma^{\prime}\left(2^{-j}\right) u_{2}\right)} \\
&\left.\times e^{i 2^{m} 2^{k-m-j} \gamma^{\prime}\left(2^{-j}\right) \eta Q_{j}\left(-y\left(x, Q_{j}^{-1}\left(u_{1}\right)\right)\right)} e^{-i 2^{m} 2^{k-m-j} \gamma^{\prime}\left(2^{-j}\right) \eta Q_{j}\left(-y\left(x, Q_{j}^{-1}\left(u_{2}\right)\right)\right)} \mathrm{d} u_{1} \mathrm{~d} u_{2}\right] \mathrm{d} x .
\end{aligned}
$$

By changing the variables $u_{1}:=v+\tau, u_{2}:=v$ and $x:=x-\gamma^{\prime}\left(2^{-j}\right) v$, which can be further written as

$$
\int_{-\infty}^{\infty} \int_{-\infty}^{\infty} W_{\tau}(x)\left(\int_{-\infty}^{\infty} K_{\tau, x}(v) e^{i 2^{m} 2^{k-m-j} \gamma^{\prime}\left(2^{-j}\right) \eta P_{\tau, x}(v)} \mathrm{d} v\right) \mathrm{d} x \mathrm{~d} \tau
$$

where

$$
\left\{\begin{array}{l}
K_{\tau, x}(v):=\mathcal{K}\left(x-\gamma^{\prime}\left(2^{-j}\right) v, v+\tau\right) \cdot \overline{\mathcal{K}\left(x-\gamma^{\prime}\left(2^{-j}\right) v, v\right)} \\
W_{\tau}(x):=\varpi\left(2^{k-j} x+2^{k-m-j} r+2^{m} 2^{k-m-j} \gamma^{\prime}\left(2^{-j}\right) \tau\right) \cdot \overline{\varpi\left(2^{k-j} x+2^{k-m-j} r\right)} \\
P_{\tau, x}(v):=Q_{j}\left(-y\left(x-\gamma^{\prime}\left(2^{-j}\right) v, Q_{j}^{-1}(v+\tau)\right)\right)-Q_{j}\left(-y\left(x-\gamma^{\prime}\left(2^{-j}\right) v, Q_{j}^{-1}(v)\right)\right) .
\end{array}\right.
$$

From the definition of $y(x, t)$ in (5.67), we have $-y\left(x-\gamma^{\prime}\left(2^{-j}\right) v, Q_{j}^{-1}(v)\right)=-x+Q_{j}^{-1}(v)$ and $-y\left(x-\gamma^{\prime}\left(2^{-j}\right) v, Q_{j}^{-1}(v+\tau)\right)=-x-\gamma^{\prime}\left(2^{-j}\right) \tau+Q_{j}^{-1}(v+\tau)$. Furthermore, let $P(x, v):=Q_{j}\left(-x+Q_{j}^{-1}(v)\right)$. Then

$$
P_{\tau, x}(v)=P\left(x+\gamma^{\prime}\left(2^{-j}\right) \tau, v+\tau\right)-P(x, v) .
$$

We now turn to $\|T(\varpi)\|_{L^{2}(\mathbb{R})}^{2}$. Indeed, from the definition of $\mathcal{K}$ in $(5.68)$, we have $Q_{j}^{-1}(v) \in$ supp $\rho$, and $|v| \lesssim 1$ for $j$ large enough. At the same time, we have $|v+\tau| \lesssim 1$. Therefore, there exists a positive constant $\epsilon_{0}>1$ such that

$$
|\tau| \leq \epsilon_{0}
$$

Furthermore, we also have $\left|K_{\tau, x}(v)\right| \lesssim 1$, which leads to

$$
\left|\int_{-\infty}^{\infty} K_{\tau, x}(v) e^{i 2^{m} 2^{k-m-j} \gamma^{\prime}\left(2^{-j}\right) \eta P_{\tau, x}(v)} \mathrm{d} v\right| \lesssim 1 .
$$

We now consider two cases, i.e., $\frac{\gamma^{\prime}\left(2^{-j}\right)}{|x|} \geq \frac{1}{2 \epsilon_{0}}$ and $\frac{\gamma^{\prime}\left(2^{-j}\right)}{|x|}<\frac{1}{2 \epsilon_{0}}$. For the first case, note that $\frac{\gamma^{\prime}\left(2^{-j}\right)}{2^{m+j-k}} \in \operatorname{supp} \phi$; it is easy to see that

$$
\|T(\varpi)\|_{L^{2}(\mathbb{R})}^{2} \lesssim 2^{m+j-k}\|\varpi\|_{L^{\infty}(\mathbb{R})}^{2} .
$$

For the second case, i.e., $\frac{\gamma^{\prime}\left(2^{-j}\right)}{|x|}<\frac{1}{2 \epsilon_{0}}$. We need the following Lemma 5.5 and Proposition 5.6, 
Lemma 5.5. [33. Lemma 2.1] Suppose $\phi$ is real-valued and smooth in $(a, b)$ and that both $\left|\phi^{\prime}(x)\right| \geq$ $\sigma_{1}$ and $\left|\phi^{\prime \prime}(x)\right| \leq \sigma_{2}$ for any $x \in(a, b)$. Then,

$$
\left|\int_{a}^{b} e^{i \phi(t)} d t\right| \leq \frac{2}{\sigma_{1}}+(b-a) \frac{\sigma_{2}}{\sigma_{1}^{2}}
$$

Proposition 5.6. Let all of the variables $x, \tau, v$, and $\eta$ be the same as in (5.70). If $\frac{\gamma^{\prime}\left(2^{-j}\right)}{|x|}<\frac{1}{2 \epsilon_{0}}$, then there exists a positive constant $C$ such that

$$
\left|\frac{d P_{\tau, x}}{d v}(v)\right| \geq C|x \| \tau| \quad \text { and } \quad\left|\frac{d^{2} P_{\tau, x}}{d^{2} v}(v)\right| \leq C|x|
$$

where $j$ is large enough and $\epsilon_{0}$ can be found in 5.71).

Let us postpone the proof of Proposition 5.6 for the moment. By Lemma 5.5, similar to the Corollary on P. 334 in Stein's book [42], with (5.74) in Proposition 5.6 and the fact that $\frac{\gamma^{\prime}\left(2^{-j}\right)}{2^{m+j-k}} \in$ supp $\phi$ and $2^{-6-\frac{2}{C_{1}}} \leq|\eta| \leq 2^{6+\frac{2}{C_{1}}}$, we have

$$
\left|\int_{-\infty}^{\infty} K_{\tau, x}(v) e^{i 2^{m} 2^{k-m-j} \gamma^{\prime}\left(2^{-j}\right) \eta P_{\tau, x}(v)} \mathrm{d} v\right| \lesssim \frac{1}{2^{m}|x||\tau|}+\frac{1}{2^{m}|x||\tau|^{2}} .
$$

From (5.72) and (5.75), we can estimate

$$
\left|\int_{-\infty}^{\infty} K_{\tau, x}(v) e^{i 2^{m} 2^{k-m-j} \gamma^{\prime}\left(2^{-j}\right) \eta P_{\tau, x}(v)} \mathrm{d} v\right| \lesssim\left(\frac{1}{2^{m}|x||\tau|}\right)^{\frac{1}{4}}+\left(\frac{1}{2^{m}|x||\tau|^{2}}\right)^{\frac{1}{4}} .
$$

Note that $|v| \lesssim 1$, from the definition of $\mathcal{K}$, we have $|x| \lesssim 1$ for $j$ large enough. From (5.71) and (5.76), we have

$$
\|T(\varpi)\|_{L^{2}(\mathbb{R})}^{2} \lesssim \int_{|\tau| \leq \epsilon_{0}} \int_{|x| \lesssim 1}\left(\frac{1}{2^{m}|x||\tau|}\right)^{\frac{1}{4}}+\left(\frac{1}{2^{m}|x||\tau|^{2}}\right)^{\frac{1}{4}} \mathrm{~d} x \mathrm{~d} \tau \cdot\|\varpi\|_{L^{\infty}(\mathbb{R})}^{2} \lesssim 2^{-\frac{m}{4}}\|\varpi\|_{L^{\infty}(\mathbb{R})}^{2} .
$$

By combining (5.73), (5.65), (5.63), (5.64), (5.58) and (5.59), we have

$$
\|\mathcal{T}(\varpi)\|_{L^{2}(\mathbb{R})}^{2} \lesssim\left(2^{m+j-k}+2^{-\frac{m}{4}}+2^{-2 m}+2^{-m}\right)\|\varpi\|_{L^{\infty}(\mathbb{R})}^{2} .
$$

By (5.55), we have

$$
\left|\int_{-\infty}^{\infty} B_{j, m}^{*}(f, g)(x) \varpi(x) \mathrm{d} x\right| \leq\left(2^{m+j-k}+2^{-\frac{m}{4}}+2^{-2 m}+2^{-m}\right)^{\frac{1}{2}}\|g\|_{L^{2}(\mathbb{R})}\|\varpi\|_{L^{\infty}(\mathbb{R})} .
$$

Let $\varpi:=\operatorname{sgn}\left(B_{j, m}^{*}(f, g)\right) \cdot \chi_{\left[2^{k-j} N, 2^{k-j}(N+1)\right]}$. We see that

$$
\left\|B_{j, m, k}^{*}(f, g) \cdot \chi_{\left[2^{k-j} N, 2^{k-j}(N+1)\right]}\right\|_{L^{1}(\mathbb{R})} \leq\left(2^{m+j-k}+2^{-\frac{m}{4}}+2^{-2 m}+2^{-m}\right)^{\frac{1}{2}}\|g\|_{L^{2}(\mathbb{R})} .
$$

Furthermore, it is clear by the definition of $\mathcal{L}\left(\chi_{\mathbb{I}} \hat{f}\right)$ that

$$
\mathcal{M} \lesssim\left\{\begin{array}{rlr}
2^{-\frac{m}{8}}\|g\|_{L^{2}(\mathbb{R})}, & \text { if } & j-k+2 m \leq 0, \\
\left(\max \left\{2^{m+j-k}, 2^{-\frac{m}{4}}\right\}\right)^{\frac{1}{2}}\|g\|_{L^{2}(\mathbb{R})}, & \text { if } & j-k+2 m>0 .
\end{array}\right.
$$

We now state the proof of Proposition 5.6 . 
Proof. By simple calculation, we have

$$
\frac{\mathrm{d} P}{\mathrm{~d} v}(x, v)=\left(Q_{j}\right)^{\prime}\left(Q_{j}^{-1}(v)-x\right) \cdot\left(Q_{j}^{-1}\right)^{\prime}(v)=\frac{\left(Q_{j}\right)^{\prime}\left(Q_{j}^{-1}(v)-x\right)}{\left(Q_{j}\right)^{\prime}\left(Q_{j}^{-1}(v)\right)} .
$$

Here, we used the fact that $\left(Q_{j}^{-1}\right)^{\prime}(v) \cdot\left(Q_{j}\right)^{\prime}\left(Q_{j}^{-1}(v)\right)=1$. Furthermore, we have

$$
\frac{\mathrm{d}^{2} P}{\mathrm{~d} x \mathrm{~d} v}(x, v)=-\frac{\left(Q_{j}\right)^{\prime \prime}\left(Q_{j}^{-1}(v)-x\right)}{\left(Q_{j}\right)^{\prime}\left(Q_{j}^{-1}(v)\right)}=-\frac{2^{-j} \gamma^{\prime \prime}\left(2^{-j}\left(Q_{j}^{-1}(v)-x\right)\right)}{\gamma^{\prime}\left(2^{-j} Q_{j}^{-1}(v)\right)} .
$$

From (5.61), we have $Q_{j}^{-1}(v)-x=t_{0}\left(\xi\left(x-\gamma^{\prime}\left(2^{-j}\right) v, Q_{j}^{-1}(v)\right), \eta\right)$, which implies that $\frac{1}{2} \leq \mid Q_{j}^{-1}(v)-$ $x \mid \leq 2$. It is also easy to see that $\frac{1}{2} \leq\left|Q_{j}^{-1}(v)\right| \leq 2$. From (2.1) and $(2.3),\left|\frac{\mathrm{d}^{2} P}{\mathrm{~d} x \mathrm{~d} v}(x, v)\right|$ can be bounded by

$$
\left|\frac{2^{-j}}{2^{-j}\left(Q_{j}^{-1}(v)-x\right)} \frac{2^{-j}\left(Q_{j}^{-1}(v)-x\right) \gamma^{\prime \prime}\left(2^{-j}\left(Q_{j}^{-1}(v)-x\right)\right)}{\gamma^{\prime}\left(2^{-j}\left(Q_{j}^{-1}(v)-x\right)\right)} \frac{\gamma^{\prime}\left(2^{-j}\left(Q_{j}^{-1}(v)-x\right)\right)}{\gamma^{\prime}\left(2^{-j} Q_{j}^{-1}(v)\right)}\right| \lesssim 1 .
$$

On the other hand, by the mean value theorem,

$$
\frac{\mathrm{d}^{2} P}{\mathrm{~d}^{2} v}(x, v)=-\frac{\gamma^{\prime}\left(2^{-j}\left(Q_{j}^{-1}(v)-x\right)\right) \gamma^{\prime}\left(2^{-j}\right) 2^{-j}}{\left(\gamma^{\prime}\left(2^{-j} Q_{j}^{-1}(v)\right)\right)^{2}}\left(\frac{\gamma^{\prime \prime}}{\gamma^{\prime}}\right)^{\prime}\left(2^{-j}\left(Q_{j}^{-1}(v)-\vartheta x\right)\right) \cdot 2^{-j} x,
$$

where $\vartheta \in[0,1]$. Noting that $\left(\frac{\gamma^{\prime \prime}}{\gamma^{\prime}}\right)^{\prime}(t)=-\left(\frac{\gamma^{\prime}}{\gamma^{\prime \prime}}\right)^{\prime}(t)\left(\frac{t \gamma^{\prime \prime}(t)}{\gamma^{\prime}(t)}\right)^{2} \frac{1}{t^{2}}$, which, together with (1.1), 2.1) and (2.3), shows that

$$
\left|\frac{\mathrm{d}^{2} P}{\mathrm{~d}^{2} v}(x, v)\right| \approx|x| .
$$

By the mean value theorem, we write

$$
\frac{\mathrm{d} P_{\tau, x}}{\mathrm{~d} v}(v)=\frac{\mathrm{d}^{2} P}{\mathrm{~d} x \mathrm{~d} v}\left(x+\vartheta_{1} \gamma^{\prime}\left(2^{-j}\right) \tau, v+\tau\right) \cdot \gamma^{\prime}\left(2^{-j}\right) \tau+\frac{\mathrm{d}^{2} P}{\mathrm{~d}^{2} v}\left(x, v+\vartheta_{2} \tau\right) \cdot \tau,
$$

where $\vartheta_{1}, \vartheta_{2} \in[0,1]$. From (5.84) and (5.86), note that $\frac{\gamma^{\prime}\left(2^{-j}\right)}{|x|}<\frac{1}{2 \epsilon_{0}}<\frac{1}{2}$; it follows that

$$
\left|\frac{\mathrm{d} P_{\tau, x}}{\mathrm{~d} v}(v)\right| \geq\left|\frac{\mathrm{d}^{2} P}{\mathrm{~d}^{2} v}\left(x, v+\vartheta_{2} \tau\right)\right| \cdot|\tau|-\left|\frac{\mathrm{d}^{2} P}{\mathrm{~d} x \mathrm{~d} v}\left(x+\vartheta_{1} \gamma^{\prime}\left(2^{-j}\right) \tau, v+\tau\right)\right| \cdot \gamma^{\prime}\left(2^{-j}\right)|\tau| \gtrsim \frac{|x \| \tau|}{2} .
$$

This is the desired result regarding $\left|\frac{\mathrm{d} P_{\tau, x}}{\mathrm{~d} v}(v)\right|$. For $\left|\frac{\mathrm{d}^{2} P_{\tau, x}}{\mathrm{~d}^{2} v}(v)\right|$, from $\underline{5.86)}$ and $\frac{\gamma^{\prime}\left(2^{-j}\right)}{|x|}<\frac{1}{2},|\tau| \lesssim 1$, it implies

$$
\left|\frac{\mathrm{d}^{2} P_{\tau, x}}{\mathrm{~d}^{2} v}(v)\right| \leq\left|\frac{\mathrm{d}^{2} P}{\mathrm{~d}^{2} v}\left(x+\gamma^{\prime}\left(2^{-j}\right) \tau, v+\tau\right)\right|+\left|\frac{\mathrm{d}^{2} P}{\mathrm{~d}^{2} v}(x, v)\right| \lesssim|x| .
$$

This finishes the proof of Proposition 5.6.

Step 3: Estimates for 5.47

From (5.53) and (5.81), note that $\sigma=2^{-\frac{m}{16}}$ and $\left\|\chi_{\mathbb{I}} \hat{f}\right\|_{L^{2}(\mathbb{I})} \leq\|f\|_{L^{2}(\mathbb{R})}$; by Lemma 5.4 and the definition of $\mathcal{L}\left(\chi_{\mathbb{I}} \hat{f}\right)$, for any $\chi_{\mathbb{I}} \hat{f} \in L^{2}(\mathbb{I})$, it is easy to obtain (5.47). 


\subsection{Proof of proposition 5.2}

In Subsection 5.1, we obtained (see (5.16)

$$
\left\|B_{j, m, k}^{*}(f, g) \cdot \chi_{\left[2^{k-j} N, 2^{k-j}(N+1)\right]}\right\|_{L^{1}(\mathbb{R})} \lesssim 2^{-\frac{2 m+j-k}{6}}\|f\|_{L^{2}(\mathbb{R})}\|g\|_{L^{2}(\mathbb{R})} .
$$

In Subsection 5.2, we bounded $\left\|B_{j, m, k}^{*}(f, g) \cdot \chi_{\left[2^{k-j}, 2^{k-j}(N+1)\right]}\right\|_{L^{1}(\mathbb{R})}($ see 5.47$)$ by

$$
\left\{\begin{array}{ccc}
2^{-\frac{m}{16}}\|f\|_{L^{2}(\mathbb{R})}\|g\|_{L^{2}(\mathbb{R})}, & \text { if } & j-k+2 m \leq 0, \\
\Lambda_{j, m, k}\|f\|_{L^{2}(\mathbb{R})}\|g\|_{L^{2}(\mathbb{R})}, & \text { if } & j-k+2 m>0,
\end{array}\right.
$$

where $\Lambda_{j, m, k}:=\max \left\{2^{\frac{15 m}{16}+\frac{j}{2}-\frac{k}{2}}, 2^{\frac{m}{16}}\left(\max \left\{2^{m+j-k}, 2^{-\frac{m}{4}}\right\}\right)^{\frac{1}{2}}\right\}$. Our goal is to prove that (see Proposition 5.2. (5.15)

$$
\left\|B_{j, m, k}^{*}(f, g) \cdot \chi_{\left[2^{k-j} N, 2^{k-j}(N+1)\right]}\right\|_{L^{1}(\mathbb{R})} \lesssim 2^{-\varepsilon_{0}^{\prime} m}\|f\|_{L^{2}(\mathbb{R})}\|g\|_{L^{2}(\mathbb{R})} .
$$

Indeed, for the case that $j-k+2 m \leq 0,(5.90)$ is true with $\varepsilon_{0}^{\prime}:=\frac{1}{16}$. For the case that $j-k+2 m>0$, we have

$$
\left\|B_{j, m, k}^{*}(f, g) \cdot \chi_{\left[2^{k-j} N, 2^{k-j}(N+1)\right]}\right\|_{L^{1}(\mathbb{R})} \lesssim 2^{\frac{15 m}{16}+\frac{j}{2}-\frac{k}{2}}\|f\|_{L^{2}(\mathbb{R})}\|g\|_{L^{2}(\mathbb{R})} \cdot
$$

Thus, combined with 5.89), we have $\left\|B_{j, m}^{*}(f, g) \cdot \chi_{\left(\frac{2^{m}}{\gamma^{\prime}\left(2^{-j}\right)} N, \frac{2^{m}}{\gamma^{\prime}\left(2^{-j}\right)}(N+1)\right)}\right\|_{L^{1}(\mathbb{R})}$ is bounded by

$$
\left(2^{\frac{15 m}{16}+\frac{j}{2}-\frac{k}{2}}\right)^{\frac{1}{4}} \cdot\left(2^{-\frac{2 m+j-k}{6}}\right)^{\frac{3}{4}}\|f\|_{L^{2}(\mathbb{R})}\|g\|_{L^{2}(\mathbb{R})} \lesssim 2^{-\frac{m}{64}}\|f\|_{L^{2}(\mathbb{R})}\|g\|_{L^{2}(\mathbb{R})} .
$$

This is (5.90) with $\varepsilon_{0}^{\prime}:=\frac{1}{64}$. These are the proof of Proposition 5.2 .

We obtain the $L^{2}(\mathbb{R}) \times L^{2}(\mathbb{R}) \rightarrow L^{1}(\mathbb{R})$ estimate for $H_{\gamma}^{3}(f, g)$.

\section{Weak- $L^{p}(\mathbb{R}) \times L^{q}(\mathbb{R}) \rightarrow L^{r}(\mathbb{R})$ boundedness of $H_{m}(f, g)$}

We begin with a lemma which can be found in [1, Lemma 5.4]:

Lemma 6.1. [1] Lemma 5.4] Let $0<p<\infty$ and $A>0$. Then, the following statements are equivalent:

(i) $\|f\|_{L^{p, \infty}(\mathbb{R})} \leq A$.

(ii) For every Lebesgue measurable set $E$ with $0<|E|<\infty$, there exists a subset $E^{\prime} \subset E$ with $\left|E^{\prime}\right| \geq \frac{|E|}{2}$ such that $\left|\left\langle f, \chi_{E^{\prime}}\right\rangle\right| \lesssim A|E|^{\frac{1}{p^{\prime}}}$.

Recall that $\psi_{\lambda}(\xi)=2^{\lambda} \psi\left(2^{\lambda} \xi\right)$; we rewrite $H_{\gamma}^{3}(f, g)$ as

$$
H_{\gamma}^{3}(f, g)(x)=\sum_{j \in \mathbb{Z}} \sum_{m \in \mathbb{N}} \sum_{k \in \mathbb{Z}} \phi\left(\frac{\gamma^{\prime}\left(2^{-j}\right)}{2^{m+j-k}}\right) \int_{-\infty}^{\infty} \check{\phi}_{m+j} * f\left(x-2^{-j} t\right) \check{\phi}_{k} * g\left(x-\gamma\left(2^{-j} t\right)\right) \rho(t) \mathrm{d} t .
$$


Let

$$
H_{m}(f, g)(x):=\sum_{j \in \mathbb{Z}} \sum_{k \in \mathbb{Z}} \phi\left(\frac{\gamma^{\prime}\left(2^{-j}\right)}{2^{m+j-k}}\right) \int_{-\infty}^{\infty} \check{\phi}_{m+j} * f\left(x-2^{-j} t\right) \check{\phi}_{k} * g\left(x-\gamma\left(2^{-j} t\right)\right) \rho(t) \mathrm{d} t .
$$

In this section, we show that

$$
\left\|H_{m}(f, g)\right\|_{L^{r, \infty}(\mathbb{R})} \lesssim m\|f\|_{L^{p}(\mathbb{R})}\|g\|_{L^{q}(\mathbb{R})}
$$

for $r>\frac{1}{2}$. We put the absolute value inside the integral and define

$$
\left|H_{m}\right|(f, g)(x):=\sum_{j \in \mathbb{Z}} \sum_{k \in \mathbb{Z}} \phi\left(\frac{\gamma^{\prime}\left(2^{-j}\right)}{2^{m+j-k}}\right) \int_{-\infty}^{\infty}\left|\check{\phi}_{m+j} * f\left(x-2^{-j} t\right) \check{\phi}_{k} * g\left(x-\gamma\left(2^{-j} t\right)\right) \rho(t)\right| \mathrm{d} t .
$$

Indeed, our goal is to obtain

$$
\left\||| H_{m} \mid(f, g)\right\|_{L^{r, \infty}(\mathbb{R})} \lesssim m\|f\|_{L^{p}(\mathbb{R})}\|g\|_{L^{q}(\mathbb{R})} .
$$

The main tool is Lemma 6.1 above. Therefore, we may assume that $f:=\chi_{F_{1}}$ and $g:=\chi_{F_{2}}$ throughout this section, where $F_{1}$ and $F_{2}$ are a Lebesgue measurable set satisfying $0<\left|F_{1}\right|,\left|F_{2}\right|<$ $\infty$. Furthermore, let us set $F_{3}$ as a Lebesgue measurable set with $0<\left|F_{3}\right|<\infty$ and define

$$
\Omega:=\left\{x \in \mathbb{R}: M \chi_{F_{1}}(x)>C \frac{\left|F_{1}\right|}{\left|F_{3}\right|}\right\} \bigcup\left\{x \in \mathbb{R}: M \chi_{F_{2}}(x)>C \frac{\left|F_{2}\right|}{\left|F_{3}\right|}\right\},
$$

where $M$ is the Hardy-Littlewood maximal function. From the weak- $(1,1)$ boundedness of the uncentered Hardy-Littlewood maximal function $M$, we may assume that $|\Omega|<\frac{\left|F_{3}\right|}{2}$ with $C$ large enough. Let $F_{3}^{\prime}:=F_{3} \backslash \Omega$; we have $\left|F_{3}^{\prime}\right|>\frac{\left|F_{3}\right|}{2}$. By Lemma 6.1, for $r>\frac{1}{2}$, it suffices to prove

$$
\left|\left\langle\left|H_{m}\right|(f, g), \chi_{E^{\prime}}\right\rangle\right| \lesssim m\left|F_{1}\right|^{\frac{1}{p}}\left|F_{2}\right|^{\frac{1}{q}}\left|F_{3}\right|^{\frac{1}{r^{\prime}}} .
$$

Recall that $\psi$ is a nonnegative Schwartz function such that $\hat{\psi}$ is supported on $\left\{t \in \mathbb{R}:|t| \leq \frac{1}{100}\right\}$ and satisfies $\hat{\psi}(0)=1$, and $\psi_{\lambda}(x)=2^{\lambda} \psi\left(2^{\lambda} x\right)$. Furthermore, let $\Omega_{\lambda}:=\left\{x \in \Omega: \operatorname{dist}\left(x, \Omega^{\complement}\right) \geq 2^{-\lambda}\right\}$ and $\tilde{\psi}_{\lambda}:=\chi_{\Omega_{\lambda}^{C}} * \psi_{\lambda}$. Therefore, we may split $\check{\phi}_{m+j} * f$ as:

$$
F_{m, j}(x, t):=\left(\tilde{\psi}_{m+j} \cdot \check{\phi}_{m+j} * f\right)\left(x-2^{-j} t\right) \quad \text { and } \quad F_{m, j}^{\complement}(x, t):=\left(\left(1-\tilde{\psi}_{m+j}\right) \cdot \check{\phi}_{m+j} * f\right)\left(x-2^{-j} t\right) \text {. }
$$

Similarly, we split $\check{\phi}_{k} * g$ as:

$$
G_{k, j}(x, t):=\left(\tilde{\psi}_{k} \cdot \check{\phi}_{k} * g\right)\left(x-\gamma\left(2^{-j} t\right)\right) \quad \text { and } \quad G_{k, j}^{C}(x, t):=\left(\left(1-\tilde{\psi}_{k}\right) \cdot \breve{\phi}_{k} * g\right)\left(x-\gamma\left(2^{-j} t\right)\right) \text {. }
$$

Then, $\left|H_{m}\right|(f, g)$ can be split into three error terms:

$$
\left\{\begin{array}{l}
\left|H_{m}^{1}\right|(f, g)(x):=\sum_{j \in \mathbb{Z}} \sum_{k \in \mathbb{Z}} \phi\left(\frac{\gamma^{\prime}\left(2^{-j}\right)}{2^{m+j-k}}\right) \int_{-\infty}^{\infty}\left|F_{m, j}^{C}(x, t) G_{k, j}(x, t) \rho(t)\right| \mathrm{d} t ; \\
\left|H_{m}^{2}\right|(f, g)(x):=\sum_{j \in \mathbb{Z}} \sum_{k \in \mathbb{Z}} \phi\left(\frac{\gamma^{\prime}\left(2^{-j}\right)}{2^{m+j-k}}\right) \int_{-\infty}^{\infty}\left|F_{m, j}^{C}(x, t) G_{k, j}^{C}(x, t) \rho(t)\right| \mathrm{d} t ; \\
\left|H_{m}^{3}\right|(f, g)(x):=\sum_{j \in \mathbb{Z}} \sum_{k \in \mathbb{Z}} \phi\left(\frac{\gamma^{\prime}\left(2^{-j}\right)}{2^{m+j-k}}\right) \int_{-\infty}^{\infty}\left|F_{m, j}(x, t) G_{k, j}^{C}(x, t) \rho(t)\right| \mathrm{d} t,
\end{array}\right.
$$

and a major term:

$$
\left|H_{m}^{4}\right|(f, g)(x):=\sum_{j \in \mathbb{Z}} \sum_{k \in \mathbb{Z}} \phi\left(\frac{\gamma^{\prime}\left(2^{-j}\right)}{2^{m+j-k}}\right) \int_{-\infty}^{\infty}\left|F_{m, j}(x, t) G_{k, j}(x, t) \rho(t)\right| \mathrm{d} t .
$$




\subsection{Error terms $\left|H_{m}^{1}\right|(f, g),\left|H_{m}^{2}\right|(f, g),\left|H_{m}^{3}\right|(f, g)$}

In this subsection, we want to prove that

$$
\left|\left\langle\left|H_{m}^{i}\right|(f, g), \chi_{E^{\prime}}\right\rangle\right| \lesssim m\left|F_{1}\right|^{\frac{1}{p}}\left|F_{2}\right|^{\frac{1}{q}}\left|F_{3}\right|^{\frac{1}{r^{\prime}}}
$$

for all $i \in\{1,2,3\}$ and $r>\frac{1}{2}$.

We will set up (6.10) for $\left|H_{m}^{1}\right|(f, g)$. The proofs for $\left|H_{m}^{2}\right|(f, g)$ and $\left|H_{m}^{3}\right|(f, g)$ are similar. Let $K \in \mathbb{N}$ be large enough; for any $x, y \in \mathbb{R}$ and any Lebesgue measurable set $E \subset \mathbb{R}$, define

$$
\delta_{j, K}(x, y):=\frac{1}{\left(1+2^{j+m}|x-y|\right)^{K}} \quad \text { and } \quad \delta_{j, K}(x, E):=\frac{1}{\left(1+2^{j+m} \operatorname{dist}(x, E)\right)^{K}} .
$$

Noting that $\tilde{\psi}_{m+j}\left(x-2^{-j} t\right)=\int_{\Omega_{j+m}^{C}} 2^{j+m} \psi\left(2^{j+m}\left(x-2^{-j} t-y\right)\right) \mathrm{d} y$ and $\int_{\Omega_{j+m}^{C}} \cup \Omega_{j+m} 2^{j+m} \psi\left(2^{j+m}(x-\right.$ $\left.\left.2^{-j} t-y\right)\right) \mathrm{d} y=1$ since $\hat{\psi}(0)=1$, we have $\left(1-\tilde{\psi}_{m+j}\right)\left(x-2^{-j} t\right)=\int_{\Omega_{j+m}} 2^{j+m} \psi\left(2^{j+m}\left(x-2^{-j} t-y\right)\right) \mathrm{d} y \lesssim$ $\int_{\Omega_{j+m}} 2^{j+m} \delta_{j, K}\left(x-2^{-j} t, y\right) \mathrm{d} y$. Therefore, $\left|\left\langle\left|H_{m}^{1}\right|(f, g), \chi_{E^{\prime}}\right\rangle\right|$ can be bounded by

$$
\begin{aligned}
\sum_{j \in \mathbb{Z}} \sum_{k \in \mathbb{Z}} \phi\left(\frac{\gamma^{\prime}\left(2^{-j}\right)}{2^{m+j-k}}\right) \int_{\Omega^{\complement}} \int_{-\infty}^{\infty} \int_{\Omega_{j+m}} 2^{j+m} \delta_{j, K}\left(x-2^{-j} t, y\right) \\
\times\left|\check{\phi}_{m+j} * f\left(x-2^{-j} t\right) \check{\phi}_{k} * g\left(x-\gamma\left(2^{-j} t\right)\right) \rho(t)\right| \mathrm{d} y \mathrm{~d} t \mathrm{~d} x .
\end{aligned}
$$

There are two cases: $x-2^{-j} t \in \Omega^{\complement}$ and $x-2^{-j} t \in \Omega$.

Case I: $x-2^{-j} t \in \Omega^{\complement}$

It is easy to see that $M f(x)=M \chi_{F_{1}}(x) \leq 1$ for all $x \in \mathbb{R}$; from the definition of $\Omega$, it implies

$$
\left|\check{\phi}_{m+j} * f\left(x-2^{-j} t\right)\right| \lesssim\left(\frac{\left|F_{1}\right|}{\left|F_{3}\right|}\right)^{\frac{1}{p}} .
$$

Furthermore, let us set $\operatorname{tr}_{j}(t):=2^{-j} t-\gamma\left(2^{-j} t\right)$ and $u:=x-2^{-j} t$ for any given $t$, then $x-\gamma\left(2^{-j} t\right)=$ $u+\operatorname{tr}_{j}(t)$. We also have

$$
\check{\phi}_{k} * g \lesssim M \chi_{F_{2}} \quad \text { and } \quad \delta_{j, K}\left(x-2^{-j} t, y\right) \leq \delta_{j, \frac{K}{2}}\left(y, \Omega^{\complement}\right) \cdot \delta_{j, \frac{K}{2}}(u, y) .
$$

Therefore, 6.11) is dominated by

$$
\left(\frac{\left|F_{1}\right|}{\left|F_{3}\right|}\right)^{\frac{1}{p}} \sum_{j \in \mathbb{Z}} \sum_{k \in \mathbb{Z}} \phi\left(\frac{\gamma^{\prime}\left(2^{-j}\right)}{2^{m+j-k}}\right) \int_{\Omega_{j+m}} \delta_{j, \frac{K}{2}}\left(y, \Omega^{\complement}\right) \int_{\Omega^{\complement}} 2^{j+m} \delta_{j, \frac{K}{2}}(u, y)\left(\int_{-\infty}^{\infty} M \chi_{F_{2}}\left(u+\operatorname{tr}_{j}(t)\right)|\rho(t)| \mathrm{d} t\right) \mathrm{d} u \mathrm{~d} y .
$$

For any given $u \in \Omega^{\complement}$, let $\tau:=u+\operatorname{tr}_{j}(t)$. Then, for $j>0$ large enough, we have

$$
\mathrm{d} t \leq \frac{\mathrm{d} \tau}{2^{-j}-2^{-j} \gamma^{\prime}\left(2 \cdot 2^{-j}\right)} \lesssim 2^{j} \mathrm{~d} \tau
$$

On the other hand, note that $\gamma(t)$ and $\frac{\gamma(t)}{t}$ are increasing on $(0, \infty)$ and $(2.4)$; we have

$$
\left|2^{-j} t-\gamma\left(2^{-j} t\right)\right| \leq 2 \cdot 2^{-j}+2^{1+\frac{2}{C_{1}}} \gamma\left(2^{-j}\right) \lesssim 2^{-j} .
$$


Therefore, by the fact that $\left(M \chi_{F_{2}}\right)^{\frac{1}{q}}$ is an $A_{1}$ weight, we conclude that

$$
\int_{-\infty}^{\infty} M \chi_{F_{2}}\left(u+\operatorname{tr}_{j}(t)\right)|\rho(t)| \mathrm{d} t \lesssim 2^{j} \int_{u-2^{-j}}^{u+2^{-j}}\left(M \chi_{F_{2}}(\tau)\right)^{\frac{1}{q}} \mathrm{~d} \tau \lesssim\left(M \chi_{F_{2}}(u)\right)^{\frac{1}{q}} .
$$

Note that $u \in \Omega^{\complement}$, which further implies that

$$
\int_{-\infty}^{\infty} M \chi_{F_{2}}\left(u+\operatorname{tr}_{j}(t)\right)|\rho(t)| \mathrm{d} t \lesssim\left(\frac{\left|F_{2}\right|}{\left|F_{3}\right|}\right)^{\frac{1}{q}} .
$$

Then, 6.11 can be bounded by

$$
\left(\frac{\left|F_{1}\right|}{\left|F_{3}\right|}\right)^{\frac{1}{p}}\left(\frac{\left|F_{2}\right|}{\left|F_{3}\right|}\right)^{\frac{1}{q}} \sum_{j \in \mathbb{Z}} \sum_{k \in \mathbb{Z}} \phi\left(\frac{\gamma^{\prime}\left(2^{-j}\right)}{2^{m+j-k}}\right) \int_{\Omega_{j+m}} \delta_{j, \frac{K}{2}}\left(y, \Omega^{\complement}\right) \int_{\Omega^{\complement}} 2^{j+m} \delta_{j, \frac{K}{2}}(u, y) \mathrm{d} u \mathrm{~d} y .
$$

When $j<0$ and $|j|$ are large enough, note that $\frac{\gamma(t)}{t}$ is strictly increasing on $(0, \infty)$ and $\gamma^{\prime}(0)=0$; we have $\frac{\gamma\left(2^{-j}\right)}{2^{-j}}>\frac{\gamma\left(J_{0}\right)}{J_{0}}$ with $\frac{\gamma\left(J_{0}\right)}{J_{0}}>2^{2+\frac{2}{C_{1}}} /\left(1+\frac{1}{2 C_{2}}\right)$ for $|j|$ large enough. From (2.4) and (2.2), we have

$$
\mathrm{d} t \leq \frac{\mathrm{d} \tau}{\left|\frac{2^{-j} t \gamma^{\prime}\left(2^{-j} t\right)}{\gamma\left(2^{-j} t\right)} \cdot \frac{\gamma\left(2^{-j} t\right)}{t}\right|-2^{-j}} \leq \frac{\mathrm{d} \tau}{\left(1+\frac{1}{2 C_{2}}\right) 2^{-2-\frac{2}{C_{1}}} \gamma\left(2^{-j}\right)-\frac{J_{0} \gamma\left(2^{-j}\right)}{\gamma\left(J_{0}\right)}} \lesssim \frac{\mathrm{d} \tau}{\gamma\left(2^{-j}\right)} .
$$

It is also easy to see that $\left|2^{-j} t-\gamma\left(2^{-j} t\right)\right| \lesssim \gamma\left(2^{-j}\right)$. Therefore, we can control 6.11) by 6.13, as in the case $j>0$.

As in (3.5) and noting that $\int_{\Omega} \mathrm{C} \frac{2^{j+m}}{\left(1+2^{j+m}|u-y|\right)^{\frac{K}{2}}} \mathrm{~d} u \lesssim 1,(6.13)$ can be bounded by

$$
\begin{aligned}
& \left(\frac{\left|F_{1}\right|}{\left|F_{3}\right|}\right)^{\frac{1}{p}}\left(\frac{\left|F_{2}\right|}{\left|F_{3}\right|}\right)^{\frac{1}{q}} \sum_{j \in \mathbb{Z}} \int_{\Omega_{j+m}} \frac{1}{\left(1+2^{j+m} \operatorname{dist}\left(y, \Omega^{\complement}\right)\right)^{\frac{K}{2}}} \int_{\Omega^{\complement}} \frac{2^{j+m}}{\left(1+2^{j+m}|u-y|\right)^{\frac{K}{2}}} \mathrm{~d} u \mathrm{~d} y \\
\leq & \left(\frac{\left|F_{1}\right|}{\left|F_{3}\right|}\right)^{\frac{1}{p}}\left(\frac{\left|F_{2}\right|}{\left|F_{3}\right|}\right)^{\frac{1}{q}} \sum_{j \in \mathbb{Z}} \int_{\Omega_{j+m}} \frac{1}{\left(1+2^{j+m} \operatorname{dist}\left(y, \Omega^{\complement}\right)\right)^{\frac{K}{2}}} \mathrm{~d} y \leq\left(\frac{\left|F_{1}\right|}{\left|F_{3}\right|}\right)^{\frac{1}{p}}\left(\frac{\left|F_{2}\right|}{\left|F_{3}\right|}\right)^{\frac{1}{q}}|\Omega| .
\end{aligned}
$$

This is the desired estimate, since $|\Omega|<\frac{\left|F_{3}\right|}{2}$. The last inequality above follows from the fact

$$
\begin{aligned}
\sum_{j \in \mathbb{Z}} \int_{\Omega_{j+m}} \frac{1}{\left(1+2^{j+m} \operatorname{dist}\left(y, \Omega^{\complement}\right)\right)^{\frac{K}{2}}} \mathrm{~d} y & \leq \int_{\Omega} \sum_{l \in \mathbb{N}} \sum_{j \in \mathbb{Z}: 2^{-j-m+l} \leq \operatorname{dist}\left(y, \Omega^{\complement}\right) \leq 2^{-j-m+l+1}} \frac{1}{\left(1+2^{j+m} \operatorname{dist}\left(y, \Omega^{\complement}\right)\right)^{\frac{K}{2}}} \mathrm{~d} y \\
& \lesssim|\Omega| \sum_{l \in \mathbb{N}} \frac{1}{\left(1+2^{l}\right)^{\frac{K}{2}}} \lesssim|\Omega| .
\end{aligned}
$$

Here, we used the fact that $\sharp\left\{j \in \mathbb{Z}: 2^{-j-m+l} \leq \operatorname{dist}\left(y, \Omega^{\complement}\right) \leq 2^{-j-m+l+1}\right\} \lesssim 1$ for any given $y \in \Omega$ and $l, m \in \mathbb{N}$.

Case II: $x-2^{-j} t \in \Omega$

In this case, we use the Whitney decomposition theorem to the open set $\Omega$ (see, for example, [22, P. 609]). Let $\mathcal{F}$ be a collection of pairwise disjoint dyadic interval $J$ 's such that $\Omega=\bigcup_{J \in \mathcal{F}} J$, 
Then, for each $J \in \mathcal{F}$, we have $|J| \leq \operatorname{dist}\left(J, \Omega^{\complement}\right) \leq 4|J|$; thus, $10 J$ meets $\Omega^{\complement}$. Furthermore, for each $J \in \mathcal{F}$ and $i \in\{1,2\}$, we have

$$
\frac{1}{|10 J|} \int_{10 J} \chi_{F_{i}}(x) \mathrm{d} x \lesssim \frac{\left|F_{i}\right|}{\left|F_{3}\right|}
$$

We now introduce the following two lemmas which can be found in [37]:

Lemma 6.2. [37 Lemma 8.1] Let $I_{1}, I_{2}$ be two intervals in $\mathcal{F}$. Suppose that $a \in I_{1}$ and $b \in I_{2}$. If $\operatorname{dist}\left(I_{1}, I_{2}\right) \geq 100 \min \left\{\left|I_{1}\right|,\left|I_{2}\right|\right\}$, then

$$
\delta_{j, K}(a, b) \lesssim \delta_{j, \frac{K}{2}}\left(a, \Omega^{\complement}\right) \cdot \delta_{j, \frac{K}{2}}\left(b, \Omega^{\complement}\right) .
$$

Lemma 6.3. [37 Lemma 8.2] Let $I_{1}, I_{2}$ be two intervals in $\mathcal{F}$. Suppose that dist $\left(I_{1}, I_{2}\right) \leq$ $100 \min \left\{\left|I_{1}\right|,\left|I_{2}\right|\right\}$, then

$$
\frac{\left|I_{2}\right|}{2000} \leq\left|I_{1}\right| \leq 2000\left|I_{2}\right|
$$

We now turn to 6.11; we rewrite it as

$$
\begin{aligned}
\sum_{j \in \mathbb{Z}} \sum_{k \in \mathbb{Z}} \phi\left(\frac{\gamma^{\prime}\left(2^{-j}\right)}{2^{m+j-k}}\right) \sum_{I_{1}, I_{2} \in \mathcal{F}} \int_{\Omega^{C}} \int_{-\infty}^{\infty} \int_{\Omega_{j+m}} 2^{j+m} \chi_{I_{1}}(y) \chi_{I_{2}}\left(x-2^{-j} t\right) \delta_{j, K}\left(x-2^{-j} t, y\right) \\
\times\left|\check{\phi}_{m+j} * f\left(x-2^{-j} t\right) \check{\phi}_{k} * g\left(x-\gamma\left(2^{-j} t\right)\right) \rho(t)\right| \mathrm{d} y \mathrm{~d} t \mathrm{~d} x .
\end{aligned}
$$

Case IIa: $\operatorname{dist}\left(I_{1}, I_{2}\right) \geq 100 \min \left\{\left|I_{1}\right|,\left|I_{2}\right|\right\}$

In this case, by Lemma 6.2, we have $\delta_{j, K}\left(x-2^{-j} t, y\right) \lesssim \delta_{j, \frac{K}{2}}\left(x-2^{-j} t, \Omega^{\complement}\right) \cdot \delta_{j, \frac{K}{2}}\left(y, \Omega^{\complement}\right)$. Then, (6.14) can be bounded by

$$
\begin{aligned}
& \sum_{j \in \mathbb{Z}} \sum_{k \in \mathbb{Z}} \phi\left(\frac{\gamma^{\prime}\left(2^{-j}\right)}{2^{m+j-k}}\right) \sum_{I_{1}, I_{2} \in \mathcal{F}} \int_{\Omega^{\complement}} \int_{-\infty}^{\infty}\left(\int_{\Omega_{j+m}} 2^{j+m} \chi_{I_{1}}(y) \delta_{j, \frac{K}{2}}\left(y, \Omega^{\complement}\right) \mathrm{d} y\right) \\
& \times \chi_{I_{2}}\left(x-2^{-j} t\right) \cdot \delta_{j, \frac{K}{2}}\left(x-2^{-j} t, \Omega^{\complement}\right)\left|\check{\phi}_{m+j} * f\left(x-2^{-j} t\right) \check{\phi}_{k} * g\left(x-\gamma\left(2^{-j} t\right)\right) \rho(t)\right| \mathrm{d} t \mathrm{~d} x .
\end{aligned}
$$

For each $x-2^{-j} t \in I_{2}$, we choose $z \in \Omega^{\complement}$ such that $\operatorname{dist}\left(x-2^{-j} t, \Omega^{\complement}\right) \approx\left|x-2^{-j} t-z\right|$. The definition of $\Omega$ implies that $\delta_{j, \frac{K}{2}}\left(x-2^{-j} t, \Omega^{\complement}\right) \cdot\left|\check{\phi}_{m+j} * f\left(x-2^{-j} t\right)\right|$ can be bounded by

$$
\begin{aligned}
& \int_{-\infty}^{\infty} f(w) \frac{2^{j+m}}{\left(1+2^{j+m}\left|x-2^{-j} t-w\right|\right)^{\frac{K}{2}}} \mathrm{~d} w \cdot \frac{1}{\left(1+2^{j+m}\left|x-2^{-j} t-z\right|\right)^{\frac{K}{2}}} \\
\lesssim & \int_{-\infty}^{\infty} f(w) \frac{2^{j+m}}{\left(1+2^{j+m}|z-w|\right)^{\frac{K}{2}}} \mathrm{~d} w \lesssim M f(z) \lesssim\left(\frac{\left|F_{1}\right|}{\left|F_{3}\right|}\right)^{\frac{1}{p}} .
\end{aligned}
$$

Furthermore, let $u:=x-2^{-j} t$; we have $x-\gamma\left(2^{-j} t\right)=u+\operatorname{tr}_{j}(t)$. Note that $\check{\phi}_{k} * g \lesssim M \chi_{F_{2}}$; we can bound 6.15 by

$$
\left(\frac{\left|F_{1}\right|}{\left|F_{3}\right|}\right)^{\frac{1}{p}} \sum_{j \in \mathbb{Z}} \sum_{k \in \mathbb{Z}} \phi\left(\frac{\gamma^{\prime}\left(2^{-j}\right)}{2^{m+j-k}}\right) \sum_{I_{1}, I_{2} \in \mathcal{F}} \int_{-\infty}^{\infty} \int_{-\infty}^{\infty}\left[\int_{\Omega_{j+m}} 2^{j+m} \chi_{I_{1}}(y) \delta_{j, \frac{K}{2}}\left(y, \Omega^{\complement}\right) \mathrm{d} y\right]
$$




$$
\times \chi_{I_{2}}(u) \cdot M \chi_{F_{2}}\left(u+\operatorname{tr}_{j}(t)\right)|\rho(t)| \mathrm{d} t \mathrm{~d} u .
$$

Note that (6.12) holds for all $j \in \mathbb{Z}$; we have

$$
\begin{aligned}
& \sum_{I_{2} \in \mathcal{F}} \int_{-\infty}^{\infty} \int_{-\infty}^{\infty} \chi_{I_{2}}(u) \cdot M \chi_{F_{2}}\left(u+\operatorname{tr}_{j}(t)\right)|\rho(t)| \mathrm{d} t \mathrm{~d} u \\
\lesssim & \sum_{I_{2} \in \mathcal{F}}\left|10 I_{2}\right| \inf _{w \in 10 I_{2}} M\left(\left(M \chi_{F_{2}}\right)^{\frac{1}{q}}\right)(w) \lesssim \sum_{I_{2} \in \mathcal{F}}\left|10 I_{2}\right| \inf _{w \in 10 I_{2}}\left(M \chi_{F_{2}}\right)^{\frac{1}{q}}(w) \lesssim|\Omega|\left(\frac{\left|F_{2}\right|}{\left|F_{3}\right|}\right)^{\frac{1}{q}} .
\end{aligned}
$$

On the other hand, $\left|I_{1}\right| \leq \operatorname{dist}\left(I_{1}, \Omega^{\complement}\right) \leq 4\left|I_{1}\right|$ implies that $\left|I_{1}\right| \leq \operatorname{dist}\left(y, \Omega^{\complement}\right) \leq 5\left|I_{1}\right|$ for all $y \in I_{1}$. Combining it with the fact that $\sharp\left\{j \in \mathbb{Z}: 2^{-j-m+l} \leq \operatorname{dist}\left(y, \Omega^{\complement}\right) \leq 2^{-j-m+l+1}\right\} \lesssim 1$ and $I_{1}$ is a dyadic interval, we obtain

$$
\begin{aligned}
& \sum_{j \in \mathbb{Z}} \sum_{I_{1} \in \mathcal{F}} \int_{\Omega_{j+m}} 2^{j+m} \chi_{I_{1}}(y) \cdot \delta_{j, \frac{K}{2}}\left(y, \Omega^{\complement}\right) \mathrm{d} y \\
\lesssim & \sum_{I_{1} \in \mathcal{F}} \int_{I_{1}} \sum_{l \in \mathbb{N}} \sum_{j \in \mathbb{Z}: 2^{-j-m+l} \leq \operatorname{dist}\left(y, \Omega^{\complement}\right.} \frac{1}{2^{-j-m+l+1}} \frac{2^{j+m}}{\left(1+2^{j+m} \cdot 2^{-j-m+l}\right)^{\frac{K}{4}}} \frac{2^{j+m}\left|I_{1}\right|}{\left(1+2^{j+m}\left|I_{1}\right|\right)^{\frac{K}{4}}} \mathrm{~d} y \\
\lesssim & \sum_{l \in \mathbb{N}} \frac{1}{\left(1+2^{l}\right)^{\frac{K}{4}}} \sum_{j \in \mathbb{Z}: \sharp\{j\} \leqslant 1} \sum_{I_{1} \in \mathcal{F}} \frac{\left.2^{j+m}\left|I_{1}\right|\right)^{\frac{K}{4}}}{(1+1 .}
\end{aligned}
$$

Therefore, for this case, we also have

$$
\left|\left\langle\left|H_{m}^{1}\right|(f, g), \chi_{E^{\prime}}\right\rangle\right| \lesssim\left(\frac{\left|F_{1}\right|}{\left|F_{3}\right|}\right)^{\frac{1}{p}}\left(\frac{\left|F_{2}\right|}{\left|F_{3}\right|}\right)^{\frac{1}{q}}|\Omega| .
$$

Case IIb: $\operatorname{dist}\left(I_{1}, I_{2}\right) \leq 100 \min \left\{\left|I_{1}\right|,\left|I_{2}\right|\right\}$

By Lemma 6.3, we have $\frac{\left|I_{2}\right|}{2000} \leq\left|I_{1}\right| \leq 2000\left|I_{2}\right|$ in this case. We may assume that $\max \left\{\left|I_{1}\right|,\left|I_{2}\right|\right\} \leq$ $2^{50} 2^{-j}$. Otherwise, we have $\left|I_{2}\right| \geq 2^{8} 2^{-j}$. Since $x-2^{-j} t \in I_{2}$ and $x \in \Omega^{\complement}$, we have

$$
\operatorname{dist}\left(x, I_{2}\right) \leq\left|x-\left(x-2^{-j} t\right)\right| \leq 2 \cdot 2^{-j} \leq \frac{\left|I_{2}\right|}{2^{7}}
$$

which further implies that $\operatorname{dist}\left(\Omega^{\complement}, I_{2}\right) \leq \frac{\left|I_{2}\right|}{2^{7}}$. This is a contradiction from $I_{2} \in \mathcal{F}$.

On the other hand, we may also assume that $\left|I_{1}\right| \geq 2^{-10} 2^{-j-m}$. If not, $y \in I_{1} \cap \Omega_{j+m}$ implies that $2^{-j-m} \leq \operatorname{dist}\left(y, \Omega^{\complement}\right) \leq 4\left|I_{1}\right| \leq 2^{-8} 2^{-j-m}$. It is a contradiction. Therefore, for any $I_{1} \in \mathcal{F}$, we have

$$
\frac{1}{2^{m+10}\left|I_{1}\right|} \leq 2^{j} \leq \frac{2^{50}}{\left|I_{1}\right|}
$$

Then, for any given $I_{1} \in \mathcal{F}$, there are at most $10 m$ many $j$ 's when $m$ is large enough.

Furthermore, for any given $I_{1} \in \mathcal{F}$, from $\frac{\left|I_{2}\right|}{2000} \leq\left|I_{1}\right| \leq 2000\left|I_{2}\right|$, the number of interval $I_{2}$ 's with $\operatorname{dist}\left(I_{1}, I_{2}\right) \leq 100 \min \left\{\left|I_{1}\right|,\left|I_{2}\right|\right\}$ is finite and independent of $m$. Let $u:=x-2^{-j} t$, then $x-\gamma\left(2^{-j} t\right)=$ $u+\operatorname{tr}_{j}(t)$. Without loss of generality, 6.14) can be controlled by

$$
\sum_{I_{1} \in \mathcal{F}} \sum_{j \in \mathbb{Z}: \frac{1}{2^{m+10}\left|I_{1}\right|} \leq 2^{j} \leq 2^{250}} \sum_{k \in \mathbb{Z}} \phi\left(\frac{\gamma^{\prime}\left(2^{-j}\right)}{2^{m+j-k}}\right) \int_{-\infty}^{\infty} \int_{-\infty}^{\infty} \int_{\Omega_{j+m}} 2^{j+m} \chi_{I_{1}}(y) \chi_{I_{1}}(u) \chi_{\Omega^{c}}\left(u+2^{-j} t\right)
$$




$$
\times \delta_{j, K}(u, y)\left|\check{\phi}_{m+j} * f(u) \cdot \check{\phi}_{k} * g\left(u+\operatorname{tr}_{j}(t)\right) \rho(t)\right| \mathrm{d} y \mathrm{~d} t \mathrm{~d} u .
$$

As in (6.16), we have $\delta_{j, \frac{K}{2}}(u, y) \cdot\left|\check{\phi}_{m+j} * f(u)\right| \lesssim\left(M \chi_{F_{1}}\right)^{\frac{1}{p}}(y)$. Noting that $\check{\phi}_{k} * g \lesssim\left(M \chi_{F_{2}}\right)^{\frac{1}{q}}$ and (3.5), we bound 6.21) by

$$
\begin{aligned}
& \sum_{I_{1} \in \mathcal{F}} \sum_{j \in \mathbb{Z}: \frac{1}{2^{m+10}\left|I_{1}\right|} \leq 2^{j} \leq \frac{2^{50}}{\left|I_{1}\right|}} \int_{-\infty}^{\infty} \int_{-\infty}^{\infty} \int_{\Omega_{j+m}} 2^{j+m} \chi_{I_{1}}(y) \chi_{I_{1}}(u) \chi_{\Omega^{\complement} \mathrm{C}}\left(u+2^{-j} t\right) \\
& \times \delta_{j, \frac{K}{2}}(u, y) \cdot\left(M \chi_{F_{1}}\right)^{\frac{1}{p}}(y) \cdot\left(M \chi_{F_{2}}\right)^{\frac{1}{q}}\left(u+\operatorname{tr}_{j}(t)\right) \cdot|\rho(t)| \mathrm{d} y \mathrm{~d} t \mathrm{~d} u .
\end{aligned}
$$

As in 6.12 , by the fact that $2^{-j} t \lesssim 2^{-j}$ for $j>0$ and $2^{-j} t \lesssim \gamma\left(2^{-j}\right)$ for $j \leq 0$, it is easy to see that

$$
\int_{-\infty}^{\infty} \chi_{\Omega^{C}}\left(u+2^{-j} t\right) \cdot\left(M \chi_{F_{2}}\right)^{\frac{1}{q}}\left(u+\operatorname{tr}_{j}(t)\right) \cdot|\rho(t)| \mathrm{d} t \lesssim\left(M \chi_{F_{2}}\right)^{\frac{1}{q}}(w)
$$

for some $w \in \Omega^{\complement}$. From the definition of $\Omega$, it implies that

$$
\int_{-\infty}^{\infty} \chi_{\Omega^{\mathrm{C}}}\left(u+2^{-j} t\right) \cdot\left(M \chi_{F_{2}}\right)^{\frac{1}{q}}\left(u+\operatorname{tr}_{j}(t)\right) \cdot|\rho(t)| \mathrm{d} t \lesssim\left(\frac{\left|F_{2}\right|}{\left|F_{3}\right|}\right)^{\frac{1}{q}} .
$$

We also have

$$
\int_{-\infty}^{\infty} 2^{j+m} \chi_{I_{1}}(u) \cdot \delta_{j, \frac{K}{2}}(u, y) \mathrm{d} u \lesssim M \chi_{I_{1}}(y) \lesssim 1 .
$$

Therefore, as in 6.18), we bound 6.22) by

$$
\begin{aligned}
& \left(\frac{\left|F_{2}\right|}{\left|F_{3}\right|}\right)^{\frac{1}{q}} \sum_{I_{1} \in \mathcal{F}} \sum_{j \in \mathbb{Z}: \frac{1}{2^{m+10}\left|I_{1}\right|} \leq 2^{j} \leq \frac{250}{\left|I_{1}\right|}} \int_{\Omega_{j+m}} \chi_{I_{1}}(y)\left(M \chi_{F_{1}}\right)^{\frac{1}{p}}(y) \mathrm{d} y \\
& \leq m\left(\frac{\left|F_{2}\right|}{\left|F_{3}\right|}\right)^{\frac{1}{q}} \sum_{I_{1} \in \mathcal{F}}\left|10 I_{1}\right| \frac{1}{\left|10 I_{1}\right|} \int_{10 I_{1}}\left(M \chi_{F_{1}}\right)^{\frac{1}{p}}(y) \mathrm{d} y \leq m|\Omega|\left(\frac{\left|F_{1}\right|}{\left|F_{3}\right|}\right)^{\frac{1}{p}}\left(\frac{\left|F_{2}\right|}{\left|F_{3}\right|}\right)^{\frac{1}{q}} .
\end{aligned}
$$

This is the desired estimate, since $|\Omega|<\frac{\left|F_{3}\right|}{2}$. Therefore, we obtain 6.10 .

\subsection{Major term $\left|H_{m}^{4}\right|(f, g)$}

In this subsection, we want to prove that

$$
\left|\left\langle\left|H_{m}^{4}\right|(f, g), \chi_{E^{\prime}}\right\rangle\right| \lesssim m\left|F_{1}\right|^{\frac{1}{p}}\left|F_{2}\right|^{\frac{1}{q}}\left|F_{3}\right|^{\frac{1}{r^{\prime}}}
$$

for $r>\frac{1}{2}$. It is easy to see that $\left|\left\langle\left|H_{m}^{4}\right|(f, g), \chi_{E^{\prime}}\right\rangle\right|$ can be bounded by

$$
\sum_{j \in \mathbb{Z}} \sum_{k \in \mathbb{Z}} \phi\left(\frac{\gamma^{\prime}\left(2^{-j}\right)}{2^{m+j-k}}\right) \int_{-\infty}^{\infty} \int_{-\infty}^{\infty}\left|\left(\tilde{\psi}_{m+j} \cdot \check{\phi}_{m+j} * f\right)\left(x-2^{-j} t\right)\left(\tilde{\psi}_{k} \cdot \check{\phi}_{k} * g\right)\left(x-\gamma\left(2^{-j} t\right)\right) \rho(t)\right| \mathrm{d} t \mathrm{~d} x .
$$

It will be bounded by $m\left|F_{1}\right|^{\frac{1}{p}}\left|F_{2}\right|^{\frac{1}{q}}\left|F_{3}\right|^{\frac{1}{r}}$. In what follows, we give the proof for the case $j>0$. The case $j \leq 0$ can be handled similarly. According to the value range of $r$, we consider the following two cases: $r \geq 1$ and $\frac{1}{2}<r<1$. The case $r \geq 1$ follows from the following proposition. 
Proposition 6.4. There exists a positive constant $C$ such that

$$
\left|\left\langle\left|H_{m}^{4}\right|(f, g), \chi_{E^{\prime}}\right\rangle\right| \leq C\left|F_{1}\right|^{\frac{1}{p}}\left|F_{2}\right|^{\frac{1}{q}}\left|F_{3}\right|^{\frac{1}{r^{\prime}}}
$$

holds for $\frac{1}{p}+\frac{1}{q}=\frac{1}{r}$, and $r \geq 1, p>1, q>1$.

Proof. Let $u:=x-\gamma\left(2^{-j} t\right)$; then $x-2^{-j} t=u-\operatorname{tr}_{j}(t)$. We can bound $\left|\left\langle\left|H_{m}^{4}\right|(f, g), \chi_{E^{\prime}}\right\rangle\right|$ by

$$
\sum_{j \in \mathbb{Z}} \sum_{k \in \mathbb{Z}} \phi\left(\frac{\gamma^{\prime}\left(2^{-j}\right)}{2^{m+j-k}}\right) \int_{-\infty}^{\infty} \int_{-\infty}^{\infty}\left|\left(\tilde{\psi}_{m+j} \cdot \check{\phi}_{m+j} * f\right)\left(u-\operatorname{tr}_{j}(t)\right)\left(\tilde{\psi}_{k} \cdot \check{\phi}_{k} * g\right)(u) \rho(t)\right| \mathrm{d} t \mathrm{~d} u .
$$

Noting that $\left|\operatorname{tr}_{j}(t)\right| \lesssim|t|$, we have

$$
\int_{-\infty}^{\infty}\left|\left(\tilde{\psi}_{m+j} \cdot \check{\phi}_{m+j} * f\right)\left(u-\operatorname{tr}_{j}(t)\right) \rho(t)\right| \mathrm{d} t \lesssim M\left(\tilde{\psi}_{m+j} \cdot \check{\phi}_{m+j} * f\right)(u) .
$$

By the Cauchy-Schwarz inequality, $\left|\left\langle\left|H_{m}^{4}\right|(f, g), \chi_{E^{\prime}}\right\rangle\right|$ can be bounded by

$$
\int_{-\infty}^{\infty}\left[\sum_{j \in T} \sum_{k \in \mathbb{Z}} \phi\left(\frac{\gamma^{\prime}\left(2^{-j}\right)}{2^{m+j-k}}\right)\left|M\left(\tilde{\psi}_{m+j} \cdot \check{\phi}_{m+j} * f\right)(u)\right|^{2}\right]^{\frac{1}{2}} \cdot\left[\sum_{j \in T} \sum_{k \in \mathbb{Z}} \phi\left(\frac{\gamma^{\prime}\left(2^{-j}\right)}{2^{m+j-k}}\right)\left|\left(\tilde{\psi}_{k} \cdot \check{\phi}_{k} * g\right)(u)\right|^{2}\right]^{\frac{1}{2}} \mathrm{~d} u .
$$

Furthermore, we also have $\left|\tilde{\psi}_{m+j}(u)\right| \lesssim \sup _{k \in \mathbb{Z}}\left|\tilde{\psi}_{k}(u)\right| \lesssim M\left(\chi_{\Omega}\right)(u) \leq 1$. By the Hölder inequality, then $\left|\left\langle\left|H_{m}^{4}\right|(f, g), \chi_{E^{\prime}}\right\rangle\right|$ is controlled by

$$
\left\|\left[\sum_{j \in T} \sum_{k \in \mathbb{Z}} \phi\left(\frac{\gamma^{\prime}\left(2^{-j}\right)}{2^{m+j-k}}\right)\left|M\left(\check{\phi}_{m+j} * f\right)\right|^{2}\right]^{\frac{1}{2}}\right\|\left\|_{L^{p}(\mathbb{R})}\right\|\left[\sum_{j \in T} \sum_{k \in \mathbb{Z}} \phi\left(\frac{\gamma^{\prime}\left(2^{-j}\right)}{2^{m+j-k}}\right)\left|\check{\phi}_{k} * g\right|^{2}\right]^{\frac{1}{2}}\left\|_{L^{q}(\mathbb{R})}\right\| M\left(\chi_{\Omega}\right) \|_{L^{r^{\prime}}(\mathbb{R})},
$$

which can be further bounded by $\|f\|_{L^{p}(\mathbb{R})}\|g\|_{L^{q}(\mathbb{R})}\left\|M\left(\chi_{\Omega}\right)\right\|_{L^{r^{\prime}(\mathbb{R})}}$ by the Fefferman-Stein inequality, the Littlewood-Paley Theory, (3.4) and (3.5). Noting that $f=\chi_{F_{1}}, g=\chi_{F_{2}}(x)$ and $|\Omega|<\frac{\left|F_{3}\right|}{2}$, from the $L^{r^{\prime}}(\mathbb{R})$ boundedness of $M$ for all $1<r^{\prime} \leq \infty,\left|\left\langle\left|H_{m}^{4}\right|(f, g), \chi_{E^{\prime}}\right\rangle\right|$ can be bounded by $\left|F_{1}\right|^{\frac{1}{p}}\left|F_{2}\right|^{\frac{1}{q}}\left|F_{3}\right|^{\frac{1}{r^{\prime}}}$. Therefore, we complete the proof of Proposition 6.4 .

The rest of this subsection is devoted to the case $\frac{1}{2}<r<1$. Let $\theta$ be a nonnegative Schwartz function such that $\hat{\theta}$ is supported on $\left\{t \in \mathbb{R}:|t| \leq 2^{-10}\right\}$ and $\hat{\theta}(0)=1, \theta_{\lambda}(x):=2^{\lambda} \theta\left(2^{\lambda} x\right), \lambda \in \mathbb{Z}$. Let $I_{n, j}:=\left[\frac{n}{2^{j}}, \frac{n+1}{2^{j}}\right]$ and $\chi_{I_{n, j}}^{*}:=\chi_{I_{n, j}} * \theta_{j+m}$. We can make a partition of unity $1=\sum_{n \in \mathbb{Z}} \chi_{I_{n, j}}^{*}(x)$. Denote

$F_{n, m, j}(x, t):=\left(\chi_{I_{n, j}}^{*} \cdot \tilde{\psi}_{m+j} \cdot \check{\phi}_{m+j} * f\right)\left(x-2^{-j} t\right) \quad$ and $\quad G_{n, m, j, k}(x, t):=\left(\chi_{I_{n, j}}^{*} \cdot \tilde{\psi}_{k} \cdot \check{\phi}_{k} * g\right)\left(x-\gamma\left(2^{-j} t\right)\right)$, and define

$$
T_{m}(f, g)(x):=\sum_{j \in \mathbb{N}} \sum_{k \in \mathbb{Z}} \phi\left(\frac{\gamma^{\prime}\left(2^{-j}\right)}{2^{m+j-k}}\right) \int_{-\infty}^{\infty}\left|\left[\sum_{n \in \mathbb{Z}} F_{n, m, j}(x, t)\right] \cdot\left[\sum_{n \in \mathbb{Z}} G_{n, m, j, k}(x, t)\right] \cdot \rho(t)\right| \mathrm{d} t .
$$

Next, we introduce the definition of a tree. 
Definition 6.5. Let $S \subset S_{0}:=\{(j, n) ; j \in \mathbb{N}, n \in \mathbb{Z}\}$. A subset $T \subset S$ is called a tree of $S$ with top $\left(j_{0}, n_{0}\right) \in S$ if $I_{n, j} \subset I_{n_{0}, j_{0}}$ for all $(j, n) \in T . T$ is called a maximal tree with top $\left(j_{0}, n_{0}\right)$ in $S$ if there is no tree in $T^{\prime} \subset S$ with the same top but strictly containing $T$.

We still need several notations. For any fixed set $S \subset S_{0}$, we abuse the notation $j \in S$ if and only if $(j, n) \in S$. For any $j \in S$, we denote $S_{j}:=\{n \in \mathbb{Z}:(j, n) \in S\}$. An operator $\Lambda_{S}[f, g]$ based on the set $S$ is defined as

$$
\sum_{j \in S} \sum_{k \in \mathbb{Z}} \phi\left(\frac{\gamma^{\prime}\left(2^{-j}\right)}{2^{m+j-k}}\right) \int_{-\infty}^{\infty} \int_{-\infty}^{\infty}\left|\left[\sum_{n \in S_{j}} F_{n, m, j}(x, t)\right] \cdot\left[\sum_{n \in S_{j}} G_{n, m, j, k}(x, t)\right] \cdot \rho(t)\right| \mathrm{d} t \mathrm{~d} x .
$$

We can use this philosophy to define other operators based on any set $U \subset S_{0}$. Then, our aim is to show that

$$
\Lambda_{S_{0}}[f, g] \lesssim m\left|F_{1}\right|^{\frac{1}{p}}\left|F_{2}\right|^{\frac{1}{q}}\left|F_{3}\right|^{\frac{1}{r^{\prime}}} .
$$

Let $T$ be a tree; we rewrite $\Lambda_{T}[f, g]$ as

$$
\sum_{j \in T} \sum_{k \in \mathbb{Z}} \phi\left(\frac{\gamma^{\prime}\left(2^{-j}\right)}{2^{m+j-k}}\right) \int_{-\infty}^{\infty} \int_{-\infty}^{\infty}\left|\left[\sum_{n \in T_{j}} f_{n, m, j}\left(u-\operatorname{tr}_{j}(t)\right)\right] \cdot\left[\sum_{n \in T_{j}} g_{n, m, j, k}(u)\right] \cdot \rho(t)\right| \mathrm{d} t \mathrm{~d} u .
$$

where $f_{n, m, j}:=\chi_{I_{n, j}}^{*} \cdot \tilde{\psi}_{m+j} \cdot \check{\phi}_{m+j} * f$ and $g_{n, m, j, k}:=\chi_{I_{n, j}}^{*} \cdot \tilde{\psi}_{k} \cdot \check{\phi}_{k} * g$. Noting that $\left|\operatorname{tr}_{j}(t)\right| \lesssim|t|$, we have

$$
\int_{-\infty}^{\infty}\left|\left[\sum_{n \in T_{j}} f_{n, m, j}\left(u-\operatorname{tr}_{j}(t)\right)\right] \rho(t)\right| \mathrm{d} t \lesssim M\left(\sum_{n \in T_{j}} f_{n, m, j}\right)(u) .
$$

By the Cauchy-Schwarz inequality and Hölder inequality, it is bounded by

$$
\left\|\left[\sum_{j \in T} \sum_{k \in \mathbb{Z}} \phi\left(\frac{\gamma^{\prime}\left(2^{-j}\right)}{2^{m+j-k}}\right)\left|M\left[\sum_{n \in T_{j}} f_{n, m, j}\right]\right|^{2}\right]^{\frac{1}{2}}\right\|\left\|_{L^{\prime}(\mathbb{R})}\right\|\left[\sum_{j \in T} \sum_{k \in \mathbb{Z}} \phi\left(\frac{\gamma^{\prime}\left(2^{-j}\right)}{2^{m+j-k}}\right)\left|\sum_{n \in T_{j}} g_{n, m, k, j}\right|^{2}\right]^{\frac{1}{2}} \|_{L^{q}(\mathbb{R})} .
$$

By the Fefferman-Stein inequality and (3.5), it is controlled by

$$
\begin{aligned}
& \quad \|\left[\sum _ { j \in T } | \sum _ { n \in T _ { j } } f _ { n , m , j } | ^ { 2 } | ^ { \frac { 1 } { 2 } } \| _ { L _ { q ^ { \prime } ( \mathbb { R } ) } } \| \left[\left.\sum_{j \in T} \sum_{k \in \mathbb{Z}} \phi\left(\frac{\gamma^{\prime}\left(2^{-j}\right)}{2^{m+j-k}}\right)\left|\sum_{n \in T_{j}} g_{n, m, k, j}\right|^{2}\right|^{\frac{1}{2}} \|_{L^{q}(\mathbb{R})}\right.\right. \\
& =:\left\|S_{1, T}(f)\right\|_{L^{q^{\prime}}(\mathbb{R})}\left\|S_{2, T}(g)\right\|_{L^{q}(\mathbb{R})},
\end{aligned}
$$

where we have defined $S_{1, T}(f)$ and $S_{2, T}(g)$ by, respectively,

$$
\left\{\begin{array}{l}
S_{1, T}(f)(x):=\left[\sum_{j \in T}\left|\sum_{n \in T_{j}} f_{n, m, j}(x)\right|^{2}\right]^{\frac{1}{2}} \\
S_{2, T}(g)(x):=\left[\sum_{j \in T} \sum_{k \in \mathbb{Z}} \phi\left(\frac{\gamma^{\prime}\left(2^{-j}\right)}{2^{m+j-k}}\right)\left|\sum_{n \in T_{j}} g_{n, m, k, j}(x)\right|^{2}\right]^{\frac{1}{2}}
\end{array}\right.
$$




\subsubsection{Sizes and BMO estimates}

For any positive integer $K$ large enough, we have

$$
\chi_{I_{n, j}}^{*}(x) \lesssim \int_{I_{n, j}} \frac{2^{j+m}}{\left(1+2^{j+m}|x-y|\right)^{K}} \mathrm{~d} y=: \chi_{I_{n, j}^{* *}}^{*}(x) \quad \text { and } \quad \chi_{I_{n, j}}^{*}(x) \lesssim \frac{1}{\left(1+2^{j+m} \operatorname{dist}\left(x, I_{n, j}\right)\right)^{K}} .
$$

Definition 6.6. Let $T \subset S$ be a tree, $I_{T}$ be the time interval of the top of tree $T$, and $D f$ be the derivative of $f$. We define the following 1-size $(T)$ and 2-size $(T)$ as

$$
\begin{aligned}
& \operatorname{1-size}(T):=\left|I_{T}\right|^{-\frac{1}{p}}\left\{\left\|\left[\sum_{j \in T}\left|\sum_{n \in T_{j}}\left(\chi_{I_{n, j}^{* *}} \cdot \chi_{\Omega_{m+j}^{C}} * \psi_{m+j} \cdot \check{\phi}_{m+j} * f\right)\right|^{2}\right]^{\frac{1}{2}}\right\|_{L^{p}(\mathbb{R})}\right. \\
& +\|\left[\left.\sum_{j \in T}\left|\sum_{n \in T_{j}}\left(\chi_{I_{n, j} *}^{*} \cdot \chi_{\Omega_{m+j}^{C}}^{C} * \psi_{m+j} \cdot(D \check{\phi})_{m+j} * f\right)\right|^{2}\right|^{\frac{1}{2}} \|_{L^{p}(\mathbb{R})}\right. \\
& +\|\left[\left.\sum_{j \in T}\left|\sum_{n \in T_{j}}\left(\chi_{I_{n, j}^{* *}}^{*} \cdot \chi_{\Omega_{m+j}^{C}}^{C} *(D \psi)_{m+j} \cdot \check{\phi}_{m+j} * f\right)\right|^{2}\right|^{\frac{1}{2}} \|_{L^{p}(\mathbb{R})}\right\},
\end{aligned}
$$

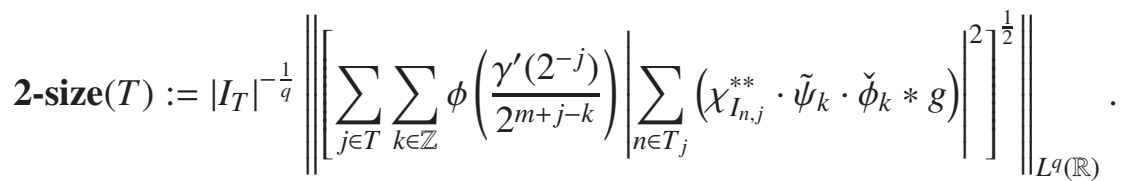

Definition 6.7. For any subset $U \subset S_{0}$ and $i \in\{1,2\}$, the $\operatorname{size}_{\mathbf{i}}(U)$ is defined as

$$
\operatorname{size}_{\mathbf{i}}(U):=\sup _{T \subset U}|\mathbf{i}-\operatorname{size}(T)|
$$

where $T \subset U$ is a tree.

Lemma 6.8. Let $\rho \in(1, \infty), M_{\rho} f:=\left(M\left(|f|^{\rho}\right)\right)^{\frac{1}{\rho}}$. Then, for any tree $T \subset S$, there exists a positive constant $C$ such that

$$
\text { 1-size }(T) \leq C \inf _{x \in I_{T}} M_{p}(M f)(x) \quad \text { and } \quad 2-\operatorname{size}(T) \leq C \inf _{x \in I_{T}} M_{q}(M g)(x) .
$$

Proof. By the Littlewood-Paley theory,

$$
\|\left[\left.\left.\left|\sum_{j \in T}\right| \sum_{n \in T_{j}}\left(\chi_{I_{n, j}^{* *}}^{*} \cdot \chi_{\Omega_{m+j}^{C}}^{C} * \psi_{m+j} \cdot \check{\phi}_{m+j} * f\right)\right|^{2}\right|^{\frac{1}{2}}\left\|_{L^{p}(\mathbb{R})} \lesssim\right\|\left[\sum_{j \in T}\left|\check{\phi}_{m+j} * f\right|^{2}\right]^{\frac{1}{2}}\left\|_{L^{p}(\mathbb{R})} \lesssim\right\| f \|_{L^{p}(\mathbb{R})} .\right.
$$


For the first part of 6.32), we split $f$ into $f \chi_{2 I_{T}}$ and $f \chi_{\left(2 I_{T}\right)}$. It is easy to see that

$$
\|\left[\left.\sum_{j \in T}\left|\sum_{n \in T_{j}}\left(\chi_{I_{n, j}^{* *}}^{*} \cdot \chi_{\Omega_{m+j}^{C}}^{C} * \psi_{m+j} \cdot \check{\phi}_{m+j} *\left(f \chi_{2 I_{T}}\right)\right)\right|^{2}\right|^{\frac{1}{2}}\left\|_{L^{p}(\mathbb{R})} \lesssim\right\| f \chi_{2 I_{T}} \|_{L^{p}(\mathbb{R})} \lesssim\left|I_{T}\right|^{\frac{1}{p}} \inf _{x \in I_{T}} M_{p} f(x),\right.
$$

and

$$
\begin{aligned}
& \|\left[\sum_{j \in T} \mid \sum_{n \in T_{j}}\left(\left.\left.\chi_{I_{n, j}^{* *}} \cdot \chi_{\Omega_{m+j}^{C}}^{C} * \psi_{m+j} \cdot \check{\phi}_{m+j} *\left(f \chi_{\left(2 I_{T}\right)} \mathrm{C}\right)\right|^{2}\right|^{\frac{1}{2}} \|_{L^{p}(\mathbb{R})}\right.\right. \\
\lesssim & \sum_{j \in T} \sum_{n \in T_{j}}\left\|\chi_{I_{n, j}^{*}}^{*} \cdot \chi_{\Omega_{m+j}^{C}} * \psi_{m+j} \cdot \check{\phi}_{m+j} *\left(f \chi_{\left(2 I_{T}\right)} \mathrm{C}\right)\right\|_{L^{p}(\mathbb{R})} .
\end{aligned}
$$

On the other hand, $\left(\chi_{I_{n, j}}^{* *} \cdot \chi_{\Omega_{m+j}^{C}} * \psi_{m+j} \cdot \breve{\phi}_{m+j} *\left(f \chi_{\left(2 I_{T}\right)} \mathrm{C}\right)\right)(x)$ can be bounded by

$$
\frac{1}{\left(1+2^{j+m} \operatorname{dist}\left(x, I_{n, j}\right)\right)^{\frac{K}{2}}} \frac{1}{\left(1+2^{j+m} \operatorname{dist}\left(I_{n, j},\left(2 I_{T}\right)^{\complement}\right)\right)^{\frac{K}{2}}} M f(x) .
$$

(6.35) is bounded by

$$
\sum_{j \in T} \sum_{n \in T_{j}}\left[\int_{-\infty}^{\infty} \frac{1}{\left(1+2^{j+m} \operatorname{dist}\left(x, I_{n, j}\right)\right)^{\frac{p K}{2}}} \frac{1}{\left(1+2^{j+m} \operatorname{dist}\left(I_{n, j},\left(2 I_{T}\right)^{C}\right)\right)^{\frac{p K}{2}}}(M f)^{p}(x) \mathrm{d} x\right]^{\frac{1}{p}} .
$$

For the integral in the square bracket above, which is further bounded by the sum of

$$
\int_{I_{T}} \frac{1}{\left(1+2^{j+m} \operatorname{dist}\left(x, I_{n, j}\right)\right)^{\frac{p K}{2}}} \frac{1}{\left(1+2^{j+m} \operatorname{dist}\left(I_{n, j},\left(2 I_{T}\right)^{\complement}\right)\right)^{\frac{p K}{2}}}(M f)^{p}(x) \mathrm{d} x=: \Theta
$$

and

$$
\sum_{l \in \mathbb{N}} \int_{2^{l+1} I_{T} \backslash 2^{l} I_{T}} \frac{1}{\left(1+2^{j+m} \operatorname{dist}\left(x, I_{n, j}\right)\right)^{\frac{p K}{2}}} \frac{1}{\left(1+2^{j+m} \operatorname{dist}\left(I_{n, j},\left(2 I_{T}\right)^{\complement}\right)\right)^{\frac{p K}{2}}}(M f)^{p}(x) \mathrm{d} x=: \sum_{l \in \mathbb{N}} \Theta_{l} .
$$

Therefore, 6.36 is bounded by

$$
\sum_{j \in T} \sum_{n \in T_{j}} \Theta^{\frac{1}{p}}+\sum_{j \in T} \sum_{n \in T_{j}}\left(\sum_{l \in \mathbb{N}} \Theta_{l}\right)^{\frac{1}{p}} .
$$

For the first part in 6.37), we have

$\frac{\frac{\left|I_{T}\right|}{\left|I_{n, j}\right|}}{\left(1+2^{j+m} \operatorname{dist}\left(I_{n, j},\left(2 I_{T}\right)^{\complement}\right)\right)^{\frac{p K}{4}}} \leq \frac{\frac{\left|I_{T}\right|}{\left|I_{n, j}\right|}}{\left(1+\frac{\left|I_{T}\right|}{\left|I_{n, j}\right|}\right)^{\frac{p K}{4}}} \lesssim 1$ and $\frac{1}{\left|I_{T}\right|} \int_{I_{T}}(M f)^{p}(x) \mathrm{d} x \lesssim \inf _{x \subseteq I_{T}}\left(M_{p}(M f)\right)^{p}(x)$. 
Thus

$$
\begin{aligned}
\Theta & \leq \frac{\left|I_{n, j}\right|}{\left(1+2^{j+m} \operatorname{dist}\left(I_{n, j},\left(2 I_{T}\right)^{\complement}\right)\right)^{\frac{p K}{2}}} \frac{\left|I_{T}\right|}{\left|I_{n, j}\right|} \frac{1}{\left|I_{T}\right|} \int_{I_{T}}(M f)^{p}(x) \mathrm{d} x \\
& \lesssim \frac{\left|I_{n, j}\right|}{\left(1+2^{j+m} \operatorname{dist}\left(I_{n, j},\left(2 I_{T}\right)^{\complement}\right)\right)^{\frac{p K}{4}}} \inf _{x \subseteq I_{T}}\left(M_{p}(M f)\right)^{p}(x) .
\end{aligned}
$$

Furthermore, we have

$$
\sum_{j \in T} \sum_{n \in T_{j}} \Theta^{\frac{1}{p}} \lesssim \sum_{j \in T} \sum_{n \in T_{j}} \frac{\left|I_{n, j}\right|^{\frac{1}{p}}}{\left(1+2^{j+m} \operatorname{dist}\left(I_{n, j},\left(2 I_{T}\right)^{\complement}\right)\right)^{\frac{K}{4}}} \inf _{x \subseteq I_{T}} M_{p}(M f)(x) .
$$

It suffices to show that

$$
\sum_{j \in T} \sum_{n \in T_{j}} \frac{\left|I_{n, j}\right|^{\frac{1}{p}}}{\left(1+2^{j+m} \operatorname{dist}\left(I_{n, j},\left(2 I_{T}\right)^{\complement}\right)\right)^{\frac{K}{4}}} \lesssim\left|I_{T}\right|^{\frac{1}{p}}
$$

Indeed, note that $\operatorname{dist}\left(I_{n, j},\left(2 I_{T}\right)^{C}\right) \gtrsim\left|I_{T}\right|$; the left-hand side of $(\underline{6.38)}$ is bounded by

$$
\sum_{j \in T} \sum_{n \in T_{j}} \frac{\left|I_{n, j}\right|^{\frac{1}{p}}}{\left(1+\frac{\left|I_{T}\right|}{\left|I_{n, j}\right|}\right)^{\frac{K}{4}}} \lesssim \sum_{l \in \mathbb{Z}_{-}} \sum_{I_{n, j}: 2^{l}\left|I_{T}\right| \leq\left|I_{n, j}\right| \leq 2^{l+1}\left|I_{T}\right|} \frac{\left(2^{l+1}\left|I_{T}\right|\right)^{\frac{1}{p}}}{\left(1+\frac{\left|I_{T}\right|}{2^{l+1}\left|I_{T}\right|}\right)^{\frac{K}{4}}} \lesssim\left|I_{T}\right|^{\frac{1}{p}} \sum_{l \in \mathbb{Z}_{-}} 2^{-l} \frac{\left(2^{l+1}\right)^{\frac{1}{p}}}{\left(1+\frac{1}{2^{l+1}}\right)^{\frac{K}{4}}} \lesssim\left|I_{T}\right|^{\frac{1}{p}} .
$$

This is the desired estimate.

For the second part in 6.37, note that

$$
\left\{\begin{array}{l}
\sum_{l \in \mathbb{N}}\left(1+\frac{2^{l}\left|I_{T}\right|}{\left|I_{n, j}\right|}\right)^{-\frac{p K}{2}} 2^{l+1} \frac{\left|I_{T}\right|}{\left|I_{n, j}\right|} \lesssim 1 ; \\
\frac{1}{2^{l+1}\left|I_{T}\right|} \int_{2^{l+1} I_{T} \backslash 2^{l} I_{T}}(M f)^{p}(x) \mathrm{d} x \lesssim \inf _{x \subseteq 2^{l+1} I_{T}}\left(M_{p}(M f)\right)^{p}(x) \lesssim \inf _{x \subseteq I_{T}}\left(M_{p}(M f)\right)^{p}(x) .
\end{array}\right.
$$

Then, $\sum_{l \in \mathbb{N}} \Theta_{l}$ can be bounded by

$$
\begin{aligned}
& \frac{\left|I_{n, j}\right|}{\left(1+2^{j+m} \operatorname{dist}\left(I_{n, j},\left(2 I_{T}\right)^{\complement}\right)\right)^{\frac{p K}{2}}} \sum_{l \in \mathbb{N}}\left(1+\frac{2^{l}\left|I_{T}\right|}{\left|I_{n, j}\right|}\right)^{-\frac{p K}{2}} 2^{l+1} \frac{\left|I_{T}\right|}{\left|I_{n, j}\right|} \frac{1}{2^{l+1}\left|I_{T}\right|} \int_{2^{l+1} I_{T} \backslash 2^{l} I_{T}}(M f)^{p}(x) \mathrm{d} x \\
& \lesssim \frac{\left|I_{n, j}\right|}{\left(1+2^{j+m} \operatorname{dist}\left(I_{n, j},\left(2 I_{T}\right)^{\complement}\right)\right)^{\frac{p K}{2}}} \inf _{x \subseteq I_{T}}\left(M_{p}(M f)\right)^{p}(x) .
\end{aligned}
$$

As in the first part in 6.37, we can obtain 6.32).

We now turn to the second part of (6.32). From (3.4) and the Littlewood-Paley theory

$$
\left\|\left[\sum_{j \in T} \sum_{k \in \mathbb{Z}} \phi\left(\frac{\gamma^{\prime}\left(2^{-j}\right)}{2^{m+j-k}}\right)\left|\sum_{n \in T_{j}}\left(\chi_{I_{n, j}^{* *}}^{*} \cdot \tilde{\psi}_{k} \cdot \check{\phi}_{k} * g\right)\right|^{2}\right]^{\frac{1}{2}}\right\|_{L^{q}(\mathbb{R})} \lesssim\left\|\left[\sum_{k \in \mathbb{Z}}\left(\check{\phi}_{k} * g\right)^{2}\right]^{\frac{1}{2}}\right\|_{L^{q}(\mathbb{R})} \lesssim\|g\|_{L^{q}(\mathbb{R})} .
$$


We also split $g$ into $g \chi_{2 I_{T}}$ and $g \chi_{\left(2 I_{T}\right)}$. As in (6.34),

$$
\|\left[\left.\sum_{j \in T} \sum_{k \in \mathbb{Z}} \phi\left(\frac{\gamma^{\prime}\left(2^{-j}\right)}{2^{m+j-k}}\right)\left|\sum_{n \in T_{j}}\left(\chi_{I_{n, j}}^{* *} \cdot \tilde{\psi}_{k} \cdot \check{\phi}_{k} *\left(g \chi_{2 I_{T}}\right)\right)\right|^{2}\right|^{\frac{1}{2}} \|_{L^{q}(\mathbb{R})} \lesssim\left|I_{T}\right|^{\frac{1}{q}} \inf _{x \in I_{T}} M_{q}(M g)(x) .\right.
$$

For the $g \chi_{\left(2 I_{T}\right)}$ part, from the fact that $\frac{\gamma^{\prime}\left(2^{-j}\right)}{2^{m+j-k}} \in \operatorname{supp} \phi, \gamma^{\prime}$ is strictly increasing on $(0, \infty), j>0$. it implies that $\frac{1}{2} \leq \frac{\gamma^{\prime}\left(2^{-j}\right)}{2^{m+j-k}} \leq \frac{\gamma^{\prime}(1)}{2^{m+j-k}}$, which further implies that $2^{m+j} \lesssim 2^{k}$. Therefore,

$$
\left(\chi_{I_{n, j}^{* *}}^{*} \cdot \tilde{\psi}_{k} \cdot \check{\phi}_{k} *\left(g \chi_{\left(2 I_{T}\right)^{C}}\right)\right)(x) \lesssim \frac{1}{\left(1+2^{j+m} \operatorname{dist}\left(x, I_{n, j}\right)\right)^{\frac{K}{2}}} \frac{1}{\left(1+2^{j+m} \operatorname{dist}\left(I_{n, j},\left(2 I_{T}\right)^{\complement}\right)\right)^{\frac{K}{2}}} M g(x) .
$$

As in (3.5), we also have $\sum_{k \in \mathbb{Z}}\left|\phi^{\frac{1}{2}}\left(\frac{\gamma^{\prime}\left(2^{-j}\right)}{2^{n+j-k}}\right)\right| \lesssim 1$. As in (6.36), we have

$$
\|\left[\left.\left.\left|\sum_{j \in T} \sum_{k \in \mathbb{Z}} \phi\left(\frac{\gamma^{\prime}\left(2^{-j}\right)}{2^{m+j-k}}\right)\right| \sum_{n \in T_{j}}\left(\chi_{I_{n, j}^{* *}}^{* *} \tilde{\psi}_{k} \cdot \check{\phi}_{k} *\left(g \chi_{\left(2 I_{T}\right)}\right)\right)\right|^{2}\right|^{\frac{1}{2}} \|_{L^{q}(\mathbb{R})} \lesssim\left|I_{T}\right|^{\frac{1}{q}} \inf _{x \in I_{T}} M_{q}(M g)(x) .\right.
$$

Therefore, we obtain 6.32). Hence, we complete the proof of Lemma 6.8.

Lemma 6.9. For the general subset $U$ of $S_{0}$ and $p \in(1, \infty)$, there exists a positive constant $C$ such that

$$
\left\|S_{1, U}(f)\right\|_{B M O} \leq C \min \left\{1, \frac{\left|F_{1}\right|}{\left|F_{3}\right|}\right\}^{\frac{1}{p}} .
$$

Proof. Let $\mathbf{J}$ be a dyadic interval of length $2^{-J}$. It suffices to bound the following formula:

$$
\inf _{c \in \mathbb{R}} \int_{\mathbf{J}} \mid\left[\left.\sum_{j \in U}\left|\sum_{n \in U_{j}}\left(\chi_{I_{n, j}}^{*} \cdot \tilde{\psi}_{m+j} \cdot \check{\phi}_{m+j} * f\right)(x)\right|^{2}\right|^{\frac{1}{2}}-c \mid \mathrm{d} x,\right.
$$

which further is bounded by a sum of the following two parts:

$$
\left\{\begin{array}{l}
J_{1}:=\int_{\mathbf{J}}\left[\sum_{j \in U: J \leq j+m}\left|\sum_{n \in U_{j}}\left(\chi_{I_{n, j}}^{*} \cdot \tilde{\psi}_{m+j} \cdot \check{\phi}_{m+j} * f\right)(x)\right|^{2}\right]^{\frac{1}{2}} \mathrm{~d} x ; \\
J_{2}:=\inf _{c \in \mathbb{R}} \int_{\mathbf{J}}\left|\left[\sum_{j \in U: J>j+m}\left|\sum_{n \in U_{j}}\left(\chi_{I_{n, j}^{*}}^{*} \cdot \tilde{\psi}_{m+j} \cdot \check{\phi}_{m+j} * f\right)(x)\right|^{2}\right]^{\frac{1}{2}}-c\right| \mathrm{d} x .
\end{array}\right.
$$

For $J_{1}$ in 6.42, we bound it by $J_{1,1}+J_{1,2}$, where

$$
\left\{\begin{array}{l}
J_{1,1}:=\int_{\mathbf{J}}\left[\sum_{j \in U: J \leq j+m}\left|\sum_{n \in U_{j}}\left(\chi_{I_{n, j}}^{*} \cdot \tilde{\psi}_{m+j} \cdot \check{\phi}_{m+j} *\left(f \chi_{2 \mathbf{J}}\right)\right)(x)\right|^{2}\right]^{\frac{1}{2}} \mathrm{~d} x ; \\
J_{1,2}:=\int_{\mathbf{J}}\left[\sum_{j \in U: J \leq j+m}\left|\sum_{n \in U_{j}}\left(\chi_{I_{n, j}}^{*} \cdot \tilde{\psi}_{m+j} \cdot \check{\phi}_{m+j} *\left(f \chi_{(2 \mathbf{J})} \mathrm{C}\right)\right)(x)\right|^{2}\right]^{\frac{1}{2}} \mathrm{~d} x .
\end{array}\right.
$$


For $J_{1,1}$, by the Hölder inequality, which is bounded by

$$
|\mathbf{J}|^{\frac{1}{p^{\prime}}} \|\left[\left.\sum_{j \in U: J \leq j+m}\left|\sum_{n \in U_{j}}\left(\chi_{I_{n, j}}^{*} \cdot \tilde{\psi}_{m+j} \cdot \check{\phi}_{m+j} *\left(f \chi_{2 \mathbf{J}}\right)\right)\right|^{2}\right|^{\frac{1}{2}} \|_{L^{p}(\mathbf{J})} .\right.
$$

As in 6.33), the above expression is bounded by

$$
|\mathbf{J}|^{\frac{1}{p^{\prime}}}\left\|f \chi_{2 \mathbf{J}}\right\|_{L^{p}(\mathbb{R})} \sup _{j \in U: J \leq j+m}\left\|\tilde{\psi}_{m+j}\right\|_{L^{\infty}(\mathbf{J})}
$$

Let $s \in \mathbb{N}$ be the least integer such that $2^{s} \mathbf{J} \cap \Omega^{\complement} \neq \emptyset$, where $2^{s} \mathbf{J}$ denotes the interval of length $2^{s}|\mathbf{J}|$ whose center is the same as that of $\mathbf{J}$, then

$$
|\mathbf{J}|^{-\frac{1}{p}}\|f \chi 2 \mathbf{J}\|_{L^{p}(\mathbb{R})} \lesssim 2^{\frac{s}{p}} \inf _{x \in 2^{s} \mathbf{J}} M_{p} f(x) \lesssim 2^{\frac{s}{p}} \min \left\{1, \frac{\left|F_{1}\right|}{\left|F_{3}\right|}\right\}^{\frac{1}{p}} .
$$

On the other hand, $|\mathbf{J}|=2^{-J} \geq 2^{-j-m}$; it implies

$$
\sup _{j \in U: J \leq j+m}\left\|\tilde{\psi}_{m+j}\right\|_{L^{\infty}(\mathbf{J})} \lesssim \sup _{j \in U: J \leq j+m} \frac{1}{\left(1+2^{j+m} \operatorname{dist}\left(x, \Omega_{j+m}^{\complement}\right)\right)^{K}} \lesssim \frac{1}{\left(1+2^{j+m} 2^{s}|\mathbf{J}|\right)^{K}} \lesssim 2^{-K s} .
$$

From 6.43), 6.44) and 6.45), we have

$$
J_{1,1} \lesssim|\mathbf{J}| \min \left\{1, \frac{\left|F_{1}\right|}{\left|F_{3}\right|}\right\}^{\frac{1}{p}} .
$$

For $J_{1,2}$, for each $x \in \mathbf{J}$, we choose $z \in \Omega^{\complement} \operatorname{such} \operatorname{dist}\left(x, \Omega^{\complement}\right) \approx|x-z|$. It implies that $\check{\phi}_{m+j} *$ $\left(f \chi_{(2 \mathbf{J})} \mathrm{C}\right)(x)$ can be bounded by

$$
\int_{(2 \mathbf{J})^{\complement}}|f(y)| 2^{j+m} \delta_{j, K}(x, y) \mathrm{d} y \leq \int_{(2 \mathbf{J})^{\complement}}|f(y)| \frac{\delta_{j, \frac{K}{2}}(z, y)}{\delta_{j, \frac{K}{2}}(z, x) \cdot \delta_{j, \frac{K}{2}}(x, y)} 2^{j+m} \delta_{j, K}(x, y) \mathrm{d} y .
$$

On the other hand, for each $x \in \mathbf{J}$ and $y \in(2 \mathbf{J})^{\complement}$, it implies that $|x-y| \geq 2^{-J-1}$. Furthermore,

$$
\check{\phi}_{m+j} *\left(f \chi_{(2 \mathbf{J})^{\complement}}\right)(x) \leq M f(z) \frac{\left(1+2^{j+m} \operatorname{dist}\left(x, \Omega^{\complement}\right)\right)^{\frac{K}{2}}}{\left(1+2^{j+m}|x-y|\right)^{\frac{K}{2}}} \leq M f(z) \frac{\left(1+2^{j+m} \operatorname{dist}\left(x, \Omega^{\complement}\right)\right)^{\frac{K}{2}}}{\left(1+2^{j+m-J-1}\right)^{\frac{K}{2}}} .
$$

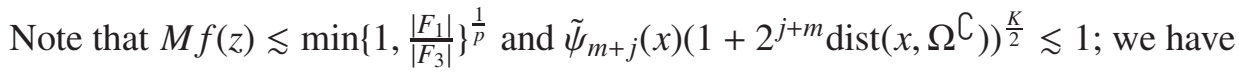

$$
J_{1,2} \lesssim \min \left\{1, \frac{\left|F_{1}\right|}{\left|F_{3}\right|}\right\}^{\frac{1}{p}} \cdot \int_{\mathbf{J}}\left[\sum_{j \in U: J \leq j+m}\left|\frac{1}{\left(1+2^{j+m-J-1}\right)^{\frac{K}{2}}}\right|^{2}\right]^{\frac{1}{2}} \mathrm{~d} x \lesssim|\mathbf{J}| \min \left\{1, \frac{\left|F_{1}\right|}{\left|F_{3}\right|}\right\}^{\frac{1}{p}}
$$


By the Hölder inequality and Poincaré inequality, $J_{2}$ in 6.42 is bounded by

$$
\begin{aligned}
& |\mathbf{J}|^{\frac{1}{2}} \inf _{c \in \mathbb{R}}\left\{\int_{\mathbf{J}} \mid\left[\left.\sum_{j \in U: J>j+m}\left|\sum_{n \in U_{j}}\left(\chi_{I_{n, j}}^{*} \cdot \tilde{\psi}_{m+j} \cdot \check{\phi}_{m+j} * f\right)(x)\right|^{2}\right|^{\frac{1}{2}}-\left.c\right|^{2} \mathrm{~d} x\right\}^{\frac{1}{2}}\right. \\
& \lesssim|\mathbf{J}|\left\{\int_{\mathbf{J}}\left|D\left[\sum_{j \in U: J>j+m}\left[\sum_{n \in U_{j}}\left(\chi_{I_{n, j}}^{*} \cdot \tilde{\psi}_{m+j} \cdot \check{\phi}_{m+j} * f\right)\right]^{2}\right](x)\right| \mathrm{d} x\right\}^{\frac{1}{2}} .
\end{aligned}
$$

Note that $\left|D\left[\sum_{n \in U_{j}}\left(\chi_{I_{n, j}^{*}}^{*} \cdot \tilde{\psi}_{m+j} \cdot \check{\phi}_{m+j} * f\right)\right]^{2}(x)\right|$ can be written as $2\left|\sum_{n \in U_{j}}\left(\chi_{I_{n, j}}^{*} \cdot \tilde{\psi}_{m+j} \cdot \check{\phi}_{m+j} * f\right)(x)\right| \cdot$ $\left|\sum_{n \in U_{j}} D\left(\chi_{I_{n, j}}^{*} \cdot \tilde{\psi}_{m+j} \cdot \check{\phi}_{m+j} * f\right)(x)\right|$. Regarding $J_{1,2}$, we have $\breve{\phi}_{m+j} * f(x) \lesssim M f(z)\left(1+2^{j+m} \operatorname{dist}\left(x, \Omega^{\complement}\right)\right)^{\frac{K}{2}}$, where $z \in \Omega^{\complement}$, which further implies that $\left|\sum_{n \in U_{j}}\left(\chi_{I_{n, j}}^{*} \cdot \tilde{\psi}_{m+j} \cdot \check{\phi}_{m+j} * f\right)(x)\right| \lesssim M f(z) \lesssim \min \left\{1, \frac{\left|F_{1}\right|}{\left|F_{3}\right|}\right\}$. At the same time, we also have that $\left|\sum_{n \in U_{j}} D\left(\chi_{I_{n, j}^{*}}^{*} \cdot \widetilde{\psi}_{m+j} \cdot \check{\phi}_{m+j} * f\right)(x)\right| \lesssim 2^{j+m} \min \left\{1, \frac{\left|F_{1}\right|}{\left|F_{3}\right|}\right\}$. Therefore, from 6.48),

$$
J_{2} \lesssim|\mathbf{J}|\left[\int_{\mathbf{J}_{j \in U: J>j+m}} 2^{j+m}\left(\min \left\{1, \frac{\left|F_{1}\right|}{\left|F_{3}\right|}\right\}\right)^{2} \mathrm{~d} x\right]^{\frac{1}{2}} \lesssim|\mathbf{J}| \min \left\{1, \frac{\left|F_{1}\right|}{\left|F_{3}\right|}\right\}^{\frac{1}{p}} .
$$

From (6.46), 6.47) and 6.49), we obtain 6.40).

Lemma 6.10. There exists a positive constant $C$ such that

$$
\left\|\chi_{I_{n, j}^{* *}}^{*} \cdot \tilde{\psi}_{m+j} \cdot \check{\phi}_{m+j} * f\right\|_{B M O} \leq C 2^{m} \operatorname{size}_{1}\left(I_{n, j}\right) .
$$

Proof. Let $\mathbf{J}$ be a dyadic interval. It suffices to bound the following formula:

$$
\inf _{c \in \mathbb{R}} \int_{\mathbf{J}}\left|\left(\chi_{I_{n, j}}^{* *} \cdot \tilde{\psi}_{m+j} \cdot \check{\phi}_{m+j} * f\right)(x)-c\right| \mathrm{d} x
$$

If $\left|I_{n, j}\right| \leq|\mathbf{J}|$, by the Hölder inequality, we have

$$
\inf _{c \in \mathbb{R}} \int_{\mathbf{J}}\left|\left(\chi_{I_{n, j}^{* *}}^{*} \cdot \tilde{\psi}_{m+j} \cdot \check{\phi}_{m+j} * f\right)(x)-c\right| \mathrm{d} x \leq\left\|\chi_{I_{n, j}^{*}}^{*} \cdot \tilde{\psi}_{m+j} \cdot \check{\phi}_{m+j} * f\right\|_{L^{p}(\mathbb{R})}|\mathbf{J}|^{\frac{1}{p^{\prime}}} \leq|\mathbf{J}| \mathbf{s i z e}_{\mathbf{1}}\left(I_{n, j}\right) .
$$

If $\left|I_{n, j}\right|>|\mathbf{J}|$, by the Poincaré inequality, we have

$$
\inf _{c \in \mathbb{R}} \int_{\mathbf{J}}\left|\left(\chi_{I_{n, j}}^{* *} \cdot \tilde{\psi}_{m+j} \cdot \check{\phi}_{m+j} * f\right)(x)-c\right| \mathrm{d} x \leq|\mathbf{J}| \int_{\mathbf{J}}\left|D\left(\chi_{I_{n, j}^{* *}}^{* *} \cdot \tilde{\psi}_{m+j} \cdot \check{\phi}_{m+j} * f\right)(x)\right| \mathrm{d} x .
$$

By the Hölder inequality, without loss of generality, the right-hand side of (6.52) can be bounded by

$$
|\mathbf{J}| 2^{j+m}\left|I_{n, j}\right|^{-\frac{1}{p}}\left\|\chi_{I_{n, j}^{* *}} \cdot \tilde{\psi}_{m+j} \cdot(D \check{\phi})_{m+j} * f\right\|_{L^{p}(\mathbb{R})}|\mathbf{J}|^{\frac{1}{p^{\prime}}}\left|I_{n, j}\right|^{\frac{1}{p}} \leq|\mathbf{J}| 2^{m} \operatorname{size}_{\mathbf{1}}\left(I_{n, j}\right) .
$$

Therefore, we obtain 6.50. 
Lemma 6.11. Let $T \subset S_{0}$ be a tree; then there exists a positive constant $C$ such that

$$
\left\|S_{1, T}(f)\right\|_{B M O} \leq C 2^{m} \text { size }_{1}(T) .
$$

Proof. Let $\mathbf{J}$ be a dyadic interval and $T_{\mathbf{J}}:=\left\{(j, n) \in T: I_{n, j} \subset 3 \mathbf{J}\right\}$. It suffices to bound the following formula:

$$
\inf _{c \in \mathbb{R}} \int_{\mathbf{J}}\left|\left[\left.\left|\sum_{j \in T}\right| \sum_{n \in T_{j}}\left(\chi_{I_{n, j}}^{*} \cdot \tilde{\psi}_{m+j} \cdot \check{\phi}_{m+j} * f\right)(x)\right|^{2}\right]^{\frac{1}{2}}-c\right| \mathrm{d} x .
$$

Furthermore, let

$$
T_{\mathbf{J}}^{1}:=\left\{(j, n) \in T \backslash T_{\mathbf{J}}:\left|I_{n, j}\right| \leq|\mathbf{J}|\right\} \quad \text { and } \quad T_{\mathbf{J}}^{2}:=\left\{(j, n) \in T \backslash T_{\mathbf{J}}:\left|I_{n, j}\right|>|\mathbf{J}|\right\} .
$$

Then, (6.54) can be bounded by a sum of the following three parts:

$$
\left\{\begin{array}{l}
J^{1}:=\int_{\mathbf{J}}\left[\sum_{j \in T_{\mathbf{J}}}\left|\sum_{n \in T_{\mathbf{J}, j}}\left(\chi_{I_{n, j}}^{*} \cdot \tilde{\psi}_{m+j} \cdot \check{\phi}_{m+j} * f\right)(x)\right|^{2}\right]^{\frac{1}{2}} \mathrm{~d} x ; \\
J^{2}:=\int_{\mathbf{J}}\left[\sum_{j \in T_{\mathbf{J}}^{1}}\left|\sum_{n \in T_{\mathbf{J}, j}^{1}}\left(\chi_{I_{n, j}^{*}}^{*} \cdot \tilde{\psi}_{m+j} \cdot \check{\phi}_{m+j} * f\right)(x)\right|^{2}\right]^{\frac{1}{2}} \mathrm{~d} x ; \\
J^{3}:=\inf _{c \in \mathbb{R}} \int_{\mathbf{J}}\left|\left[\sum_{j \in T_{\mathbf{J}}^{2}}\left(\sum_{n \in T_{\mathbf{J}, j}^{2}}\left(\chi_{I_{n, j}}^{*} \cdot \tilde{\psi}_{m+j} \cdot \check{\phi}_{m+j} * f\right)(x)\right)^{2}\right]^{\frac{1}{2}}-c\right| \mathrm{d} x .
\end{array}\right.
$$

By the Hölder inequality, $J^{1}$ can be bounded by

$$
\|\left[\left.\sum_{j \in T_{\mathbf{J}}}\left|\sum_{n \in T_{\mathbf{J}, j}}\left(\chi_{I_{n, j}}^{*} \cdot \tilde{\psi}_{m+j} \cdot \check{\phi}_{m+j} * f\right)\right|^{2}\right|^{\frac{1}{2}} \|_{L^{p}(\mathbf{J})}|\mathbf{J}|^{\frac{1}{p^{\prime}}} \leq \operatorname{size}_{\mathbf{1}}(T)|\mathbf{J}|^{\frac{1}{p}}|\mathbf{J}|^{\frac{1}{p^{\prime}}}=\operatorname{size}_{\mathbf{1}}(T)|\mathbf{J}| .\right.
$$

By the Hölder inequality, $J^{2}$ can be bounded by

$$
\begin{aligned}
& \|\left[\left.\sum_{j \in T_{\mathbf{J}}^{1}}\left|\sum_{n \in T_{\mathbf{J}, j}^{1}}\left(\chi_{I_{n, j}^{*}}^{*} \cdot \tilde{\psi}_{m+j} \cdot \check{\phi}_{m+j} * f\right)\right|^{2}\right|^{\frac{1}{2}} \|_{L^{p}(\mathbf{J})}|\mathbf{J}|^{\frac{1}{p^{\prime}}}\right. \\
& \leq \operatorname{size}_{\mathbf{1}}(T) \sum_{j \in T_{\mathbf{J}}^{1}} \sum_{n \in T_{\mathbf{J}, j}^{1}} \frac{\left|I_{n, j}\right|^{\frac{1}{p}}}{\left(1+2^{j+m} \operatorname{dist}\left(\mathbf{J}, I_{n, j}\right)\right)^{K}}|\mathbf{J}|^{\frac{1}{p^{\prime}}} \lesssim \operatorname{size}_{\mathbf{1}}(T)|\mathbf{J}| .
\end{aligned}
$$

By the Hölder inequality and Poincaré inequality, as in (6.48), we bound $J^{3}$ by

$$
\left.|\mathbf{J}|\left\{\int_{\mathbf{J}} \mid D\left[\sum_{j \in T_{\mathbf{J}}^{2}} \sum_{n \in T_{\mathbf{J}, j}^{2}} \chi_{I_{n, j}}^{*} \cdot \tilde{\psi}_{m+j} \cdot \check{\phi}_{m+j} * f\right)^{2}\right](x) \mid \mathrm{d} x\right\}^{\frac{1}{2}} .
$$


Without loss of generality, by the Cauchy-Schwarz inequality, $D\left[\sum_{n \in T_{\mathbf{J}, j}^{2}}\left(\chi_{I_{n, j}}^{*} \cdot \tilde{\psi}_{m+j} \cdot \check{\phi}_{m+j} * f\right)\right]^{2}$ can be bounded by the sum of

$$
2^{\frac{m}{2}}|\mathbf{J}|\left\{\int_{\mathbf{J}} \sum_{j \in T_{\mathbf{J}}^{2}}\left[\sum_{n \in T_{\mathbf{J}, j}^{2}} \frac{1}{\left|I_{n, j}\right|^{\frac{1}{2}}}\left|\chi_{I_{n, j}}^{*} \cdot \tilde{\psi}_{m+j} \cdot \check{\phi}_{m+j} * f(x)\right|\right]^{2} \mathrm{~d} x\right\}^{\frac{1}{2}}
$$

and

$$
2^{\frac{m}{2}}|\mathbf{J}|\left\{\int_{\mathbf{J}} \sum_{j \in T_{\mathbf{J}}^{2}}\left[\sum_{n \in T_{\mathbf{J}, j}^{2}} \frac{1}{\left|I_{n, j}\right|^{\frac{1}{2}}}\left|\chi_{I_{n, j}}^{*} \cdot \tilde{\psi}_{m+j} \cdot(D \check{\phi})_{m+j} * f(x)\right|\right]^{2} \mathrm{~d} x\right\}^{\frac{1}{2}} .
$$

It is suffices to bound (6.56). Note that

$$
\left\|\chi_{I_{n, j}^{* *}}^{*} \cdot \tilde{\psi}_{m+j} \cdot \check{\phi}_{m+j} * f\right\|_{L^{p}(\mathbb{R})} \leq\left|I_{n, j}\right|^{\frac{1}{p}} \operatorname{size}_{\mathbf{1}}\left(I_{n, j}\right)
$$

and (6.50), by interpolating, we obtain

$$
\left\|\chi_{I_{n, j}}^{* *} \cdot \tilde{\psi}_{m+j} \cdot \check{\phi}_{m+j} * f\right\|_{L^{2 p}(\mathbb{R})} \leq 2^{\frac{m}{2}}\left|I_{n, j}\right|^{\frac{1}{2 p}} \operatorname{size}_{\mathbf{1}}\left(I_{n, j}\right) .
$$

By the Hölder inequality, 6.56 can be bounded by

$$
\begin{aligned}
& 2^{\frac{m}{2}}|\mathbf{J}| \sum_{j \in T_{\mathbf{J}}^{2}} \sum_{n \in T_{\mathbf{J}, j}^{2}} \frac{1}{\left|I_{n, j}\right|^{\frac{1}{2}}} \frac{\left\|\chi_{I_{n, j}^{* *}}^{*} \tilde{\psi}_{m+j} \cdot \check{\phi}_{m+j} * f\right\|_{L^{2 p}(\mathbf{J})}|J|^{\frac{1}{2}-\frac{1}{2 p}}}{\left(1+2^{j+m} \operatorname{dist}\left(\mathbf{J}, I_{n, j}\right)\right)^{K}} \\
\leq & 2^{\frac{m}{2}}|\mathbf{J}| \sum_{j \in T_{\mathbf{J}}^{2}} \sum_{n \in T_{\mathbf{J}, j}^{2}} \frac{1}{\left|I_{n, j}\right|^{\frac{1}{2}}} \frac{2^{\frac{m}{2}}\left|I_{n, j}\right|^{\frac{1}{2 p}} \operatorname{size}_{\mathbf{1}}\left(I_{n, j}\right)|J|^{\frac{1}{2}-\frac{1}{2 p}}}{\left(1+2^{j+m} \operatorname{dist}\left(\mathbf{J}, I_{n, j}\right)\right)^{K}} \lesssim 2^{m}|\mathbf{J}| \operatorname{size}_{\mathbf{1}}(T) .
\end{aligned}
$$

The last inequality follows from

$$
\sum_{j \in T_{\mathbf{J}}^{2}} \sum_{n \in T_{\mathbf{J}, j}^{2}} \frac{\left|I_{n, j}\right|^{\frac{1}{2 p}-\frac{1}{2}}|J|^{\frac{1}{2}-\frac{1}{2 p}}}{\left(1+2^{j+m} \operatorname{dist}\left(\mathbf{J}, I_{n, j}\right)\right)^{K}} \lesssim 1,
$$

which can be found in $\mathrm{Li}[36]$. Hence, we complete the proof of Lemma 6.11

From (6.28) to 6.31, we have

$$
\left\|S_{1, T}(f)\right\|_{L^{p}(\mathbb{R})} \leq \operatorname{size}_{1}(T) \cdot\left|I_{T}\right|^{\frac{1}{p}} \quad \text { and } \quad\left\|S_{2, T}(f)\right\|_{L^{q}(\mathbb{R})} \leq \operatorname{size}_{2}(T) \cdot\left|I_{T}\right|^{\frac{1}{q}}
$$

From 6.40) in Lemma 6.9 and 6.53) Lemma 6.11, by interpolation with 6.59], respectively, we obtain

$$
\left\{\begin{array}{l}
\left\|S_{1, T}(f)\right\|_{L^{q^{\prime}}(\mathbb{R})} \lesssim\left|I_{T}\right|^{\frac{1}{q^{\prime}}} \operatorname{size}_{1}(T)^{\frac{p}{q^{\prime}}} \min \left\{1, \frac{\left|F_{1}\right|}{\left|F_{3}\right|}\right\}^{\frac{1}{p}-\frac{1}{q^{\prime}}} \\
\left\|S_{1, T}(f)\right\|_{L^{q^{\prime}}(\mathbb{R})} \lesssim\left|I_{T}\right|^{\frac{1}{q^{\prime}}} \operatorname{size}_{\mathbf{1}}(T) 2^{m\left(1-\frac{p}{q^{\prime}}\right)} .
\end{array}\right.
$$


Furthermore, we have

$$
\left\|S_{1, T}(f)\right\|_{L^{q^{\prime}}(\mathbb{R})} \lesssim\left|I_{T}\right|^{\frac{1}{q^{\prime}}} \operatorname{size}_{\mathbf{1}}^{*}(T),
$$

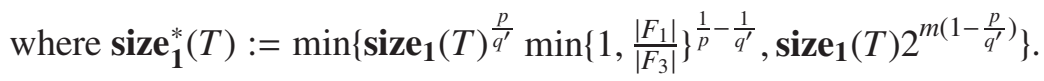

\subsubsection{The estimates for $\left|H_{m}^{4}\right|(f, g)$}

For $S \subset S_{0}$, we rewrite $\Lambda_{S}[f, g]$ as

$$
\sum_{j \in S} \sum_{k \in \mathbb{Z}} \phi\left(\frac{\gamma^{\prime}\left(2^{-j}\right)}{2^{m+j-k}}\right) \int_{-\infty}^{\infty} \int_{-\infty}^{\infty}\left|\left[\sum_{n \in S_{j}} f_{n, m, j}\left(x-\operatorname{tr}_{j}(t)\right)\right] \cdot\left[\sum_{n \in S_{j}} g_{n, m, j, k}(x)\right] \cdot \rho(t)\right| \mathrm{d} x \mathrm{~d} t .
$$

Lemma 6.12. Let $T \subset S_{0}$ be a tree and $P \subset S_{0}$ be a subset; if $T \cap P=\emptyset$ and $T$ is a maximal tree in $P \cup T$, then there exists a positive constant $C$ such that

$$
\left|\Lambda_{P \cup T}[f, g]-\Lambda_{P}[f, g]-\Lambda_{T}[f, g]\right| \leq C \operatorname{size}_{1}^{*}(P \cup T) \operatorname{size}_{2}(P \cup T)\left|I_{T}\right|,
$$

where size ${ }_{1}^{*}(P \cup T)$ is defined naturally as $\operatorname{size}_{1}^{*}(T)$.

Proof. We begin our proof by a definition and a lemma. Let

$$
d_{j}\left(S_{1}, S_{2}\right):=\left(1+2^{j+m} \operatorname{dist}\left(S_{1}, S_{2}\right)\right)^{-K} \quad \text { for any } S_{1}, S_{2} \subset S_{0},
$$

where $S_{1}$ and $S_{2}$ are defined as the union of all intervals $I_{n, j}$ with $(j, n) \in S_{1}$ and $(j, n) \in S_{2}$, respectively. As in [37, Lemma 10.1], if $T \cap P=\emptyset$, we have

$$
\left\{\begin{array}{l}
\sum_{j \in \mathbb{N}} \sum_{I:|I|=2^{-j}}|I| d_{j}\left(5 I, T_{j}\right) d_{j}\left(I, P_{j}\right) \lesssim\left|I_{T}\right| \\
\sum_{j \in \mathbb{N}} \sum_{I:|I|=2^{-j}}|I| d_{j}\left(5 I, P_{j}\right) d_{j}\left(I, T_{j}\right) \lesssim\left|I_{T}\right|
\end{array}\right.
$$

Since $T$ is a maximal tree in $P \cup T$, then the left hand side of (6.62) can be bounded by

$$
\begin{aligned}
\mathbb{A}+\mathbb{B}:= & \sum_{j \in \mathbb{N}} \sum_{k \in \mathbb{Z}} \phi\left(\frac{\gamma^{\prime}\left(2^{-j}\right)}{2^{m+j-k}}\right) \int_{-\infty}^{\infty} \int_{-\infty}^{\infty}\left|\left[\sum_{n \in P_{j}} f_{n, m, j}\left(x-\operatorname{tr}_{j}(t)\right)\right] \cdot\left[\sum_{n \in T_{j}} g_{n, m, j, k}(x)\right] \cdot \rho(t)\right| \mathrm{d} x \mathrm{~d} t \\
& +\sum_{j \in \mathbb{N}} \sum_{k \in \mathbb{Z}} \phi\left(\frac{\gamma^{\prime}\left(2^{-j}\right)}{2^{m+j-k}}\right) \int_{-\infty}^{\infty} \int_{-\infty}^{\infty}\left|\left[\sum_{n \in T_{j}} f_{n, m, j}\left(x-\operatorname{tr}_{j}(t)\right)\right] \cdot\left[\sum_{n \in P_{j}} g_{n, m, j, k}(x)\right] \cdot \rho(t)\right| \mathrm{d} x \mathrm{~d} t .
\end{aligned}
$$

We here give the estimate of $\mathbb{A} ; \mathbb{B}$ can be handled similarly. From the Hölder inequality, $\mathbb{A}$ is bounded by

$$
\sum_{j \in \mathbb{N}} \sum_{k \in \mathbb{Z}} \phi^{\frac{1}{2}}\left(\frac{\gamma^{\prime}\left(2^{-j}\right)}{2^{m+j-k}}\right) \sum_{I:|I|=2^{-j}}\left\|\int_{-\infty}^{\infty}\left|\sum_{n \in P_{j}} f_{n, m, j}\left(\cdot-\operatorname{tr}_{j}(t)\right) \cdot \rho(t)\right| \mathrm{d} t\right\|_{L^{q^{\prime}}(I)}\left\|\phi^{\frac{1}{2}}\left(\frac{\gamma^{\prime}\left(2^{-j}\right)}{2^{m+j-k}}\right) \cdot \sum_{n \in T_{j}} g_{n, m, j, k}\right\|_{L^{q}(I)} .
$$


For $x \in I$, note that $\operatorname{tr}_{j}(t) \lesssim 2^{-j}$; without loss of generality, we may write $\chi_{I_{n, j}^{*}}^{*}\left(x-\operatorname{tr}_{j}(t)\right) \lesssim$ $d_{j}\left(5 I, I_{n, j}\right) \cdot \chi_{I_{n, j}^{* *}}^{* *}\left(x-\operatorname{tr}_{j}(t)\right)$. From (6.60), $\left\|\int_{-\infty}^{\infty}\left|\sum_{n \in P_{j}} f_{n, m, j}\left(\cdot-\operatorname{tr}_{j}(t)\right) \cdot \rho(t)\right| \mathrm{d} t\right\|_{L^{q^{\prime}}(I)}$ is controlled by

$$
\begin{aligned}
& \sum_{n \in P_{j}} d_{j}\left(5 I, I_{n, j}\right)\left\|\int_{-\infty}^{\infty}\left|\left(\chi_{I_{n, j}}^{* *} \cdot \tilde{\psi}_{m+j} \cdot \check{\phi}_{m+j} * f\right)\left(\cdot-\operatorname{tr}_{j}(t)\right) \cdot \rho(t)\right| \mathrm{d} t\right\|_{L^{q^{\prime}(I)}} \\
\leq & \sum_{n \in P_{j}} d_{j}\left(5 I, I_{n, j}\right)\left\|M\left(\chi_{I_{n, j}^{* *}}^{* *} \tilde{\psi}_{m+j} \cdot \check{\phi}_{m+j} * f\right)\right\|_{L^{q^{\prime}(\mathbb{R})}} \leq d_{j}\left(5 I, P_{j}\right)|I|^{\frac{1}{q^{\prime}}} \operatorname{size}_{\mathbf{1}}^{*}(P \cup T) .
\end{aligned}
$$

It is easy to see that

$$
\left\|\phi^{\frac{1}{2}}\left(\frac{\gamma^{\prime}\left(2^{-j}\right)}{2^{m+j-k}}\right) \cdot \sum_{n \in T_{j}} g_{n, m, j, k}\right\|_{L^{q}(I)} \lesssim d_{j}\left(I, T_{j}\right)|I|^{\frac{1}{q}} \operatorname{size}_{2}(P \cup T) .
$$

As in (3.5), we have $\sum_{k \in \mathbb{Z}} \phi^{\frac{1}{2}}\left(\frac{\gamma^{\prime}\left(2^{-j}\right)}{2^{m+j-k}}\right) \lesssim 1$. This, combined with (6.64), (6.65) and (6.63), implies that Part $\mathbb{A}$ can be bounded by

$$
\sum_{j \in \mathbb{N}} \sum_{I:|I|=2^{-j}}|I| d_{j}\left(5 I, P_{j}\right) d_{j}\left(I, T_{j}\right) \operatorname{size}_{\mathbf{1}}^{*}(P \cup T) \operatorname{size}_{\mathbf{2}}(P \cup T) \lesssim \operatorname{size}_{\mathbf{1}}^{*}(P \cup T) \operatorname{size}_{\mathbf{2}}(P \cup T)\left|I_{T}\right| .
$$

Hence, we complete the proof of Lemma 6.12 .

For any $S \subset S_{0}, k \in\{1,2\}$, as in [37, Lemma 6.12], by (6.32) in Lemma 6.8, we can always split $S$ into $S_{1}$ and $S_{2}$ :

(i) $S_{1}:=\bigcup_{T \in \mathcal{F}} T$ with $\bigcup_{T \in \mathcal{F}}\left|I_{T}\right| \lesssim \frac{\left|F_{1}\right|}{\operatorname{size}_{1}(S)^{p}}$ and $\bigcup_{T \in \mathcal{F}}\left|I_{T}\right| \lesssim \frac{\left|F_{2}\right|}{\operatorname{size}_{2}(S)^{q}}$, where $T$ is maximal tree;

(ii) $S_{2}:=S \backslash S_{1}$ with $\operatorname{size}_{1}\left(S_{2}\right) \leq\left(\frac{1}{2}\right)^{\frac{1}{p}} \operatorname{size}_{1}(S)$ and $\operatorname{size}_{2}\left(S_{2}\right) \leq\left(\frac{1}{2}\right)^{\frac{1}{q}} \operatorname{size}_{2}(S)$,

which further implies that we can write $S_{0}$ as

$$
S_{0}=\bigcup_{\sigma \leq 1} S_{\sigma}
$$

where $\sigma$ ranges over positive dyadic numbers, and $S_{\sigma}$ is a union of maximal trees such that for each $T \in S_{\sigma}$, we have

$$
\operatorname{size}_{1}(T) \leq \sigma^{\frac{1}{p}}\left(\frac{\left|F_{1}\right|}{\left|F_{3}\right|}\right)^{\frac{1}{p}} \quad \text { and } \quad \operatorname{size}_{2}(T) \leq \sigma^{\frac{1}{q}}\left(\frac{\left|F_{2}\right|}{\left|F_{3}\right|}\right)^{\frac{1}{q}},
$$

and

$$
\operatorname{size}_{1}(T) \geq\left(\frac{\sigma}{2}\right)^{\frac{1}{p}}\left(\frac{\left|F_{1}\right|}{\left|F_{3}\right|}\right)^{\frac{1}{p}} \quad \text { or } \quad \operatorname{size}_{2}(T) \geq\left(\frac{\sigma}{2}\right)^{\frac{1}{q}}\left(\frac{\left|F_{2}\right|}{\left|F_{3}\right|}\right)^{\frac{1}{q}}
$$


We now turn to the proof of (6.26). Indeed, it is easy to see that

$$
\sum_{T \in S_{\sigma}}\left|I_{T}\right| \lesssim \frac{\left|F_{1}\right|}{\left(\left(\frac{\sigma}{2}\right)^{\frac{1}{p}}\left(\frac{\left|F_{1}\right|}{\left|F_{3}\right|}\right)^{\frac{1}{p}}\right)^{p}}+\frac{\left|F_{2}\right|}{\left(\left(\frac{\sigma}{2}\right)^{\frac{1}{q}}\left(\frac{\left|F_{2}\right|}{\left|F_{3}\right|}\right)^{\frac{1}{q}}\right)^{q}} \lesssim \frac{\left|F_{3}\right|}{\sigma} .
$$

Furthermore, the fact that $\Lambda_{T}[f, g]$ is bounded by 6.27), combined with the second part in 6.59] and (6.60), gives

$$
\Lambda_{T}[f, g] \lesssim\left|I_{T}\right| \operatorname{size}_{\mathbf{1}}^{*}(T) \operatorname{size}_{2}(T) .
$$

From Lemma 6.12, 6.66) and 6.70), we conclude

$$
\Lambda_{S_{0}}[f, g] \lesssim \sum_{\sigma \leq 1} \sum_{T \in S_{\sigma}}\left|I_{T}\right| \operatorname{size}_{\mathbf{1}}^{*}(T) \operatorname{size}_{2}(T)
$$

From the definition of $\operatorname{size}_{1}^{*}(T)$, 6.67) and (6.69), the above expression can be bounded by

$$
\left|F_{1}\right|^{\frac{1}{p}}\left|F_{2}\right|^{\frac{1}{q}}\left|F_{3}\right|^{\frac{1}{r^{\prime}}} \sum_{\sigma \leq 1} \sigma^{\frac{1}{q}-1} \min \left\{2^{m\left(1-\frac{p}{q^{\prime}}\right)} \sigma^{\frac{1}{p}}, \sigma^{\frac{1}{q^{\prime}}}\right\} \lesssim m\left|F_{1}\right|^{\frac{1}{p}}\left|F_{2}\right|^{\frac{1}{q}}\left|F_{3}\right|^{\frac{1}{r^{r}}}
$$

This is the desired estimate.

\section{The boundedness of $M_{\gamma}(f, g)$}

For $M_{\gamma}^{1}(f, g)$, as $H_{\gamma}^{1}(f, g)$, we write $\tilde{m}_{j}^{1}(\xi, \eta)$ as

$$
\sum_{m, n \in \mathbb{Z}_{-}} \sum_{k \in \mathbb{Z}} \sum_{u, v \in \mathbb{N}} \frac{(-i)^{u+v} 2^{m u} 2^{n v}}{u ! v !} \bar{\phi}_{u}\left(\frac{\xi}{2^{m+j}}\right) \bar{\phi}_{v}\left(\frac{\eta}{2^{k}}\right) \bar{\phi}_{v}\left(\frac{\gamma^{\prime}\left(2^{-j}\right)}{2^{n+j-k}}\right) \int_{-\infty}^{\infty} t^{u}\left(\frac{2^{j} \gamma\left(2^{-j} t\right)}{\gamma^{\prime}\left(2^{-j}\right)}\right)^{v}|\rho(t)| \mathrm{d} t .
$$

Furthermore, $M_{\gamma}^{1}(f, g)$ can be written as

$$
\begin{aligned}
M_{\gamma}^{1}(f, g)(x)=\sup _{j \in \mathbb{Z}} \mid \sum_{m, n \in \mathbb{Z}_{-}} \sum_{k \in \mathbb{Z}} \sum_{u, v \in \mathbb{N}} \frac{(-i)^{u+v} 2^{m u} 2^{n v}}{u ! v !} \bar{\phi}_{v}\left(\frac{\gamma^{\prime}\left(2^{-j}\right)}{2^{n+j-k}}\right) \\
\quad \times\left(\int_{-\infty}^{\infty} t^{u}\left(\frac{2^{j} \gamma\left(2^{-j} t\right)}{\gamma^{\prime}\left(2^{-j}\right)}\right)^{v}|\rho(t)| \mathrm{d} t\right) \check{\bar{\phi}}_{u, m+j} * f(x) \cdot \check{\bar{\phi}}_{v, k} * g(x) \mid .
\end{aligned}
$$

From [42, P. 24 Proposition], there exists a positive constant $C$ such that $\sup _{j \in \mathbb{Z}}\left|\check{\bar{\phi}}_{u, m+j} * f\right| \lesssim C^{u} M f$ and $\left|\check{\bar{\phi}}_{v, k} * g\right| \lesssim C^{v} M g$. As in (3.3) and (3.5), combining with the fact that $\sum_{m, n \in \mathbb{Z}_{-}} \sum_{u, v \in \mathbb{N}} \frac{C^{u} 2^{m u} C^{v} 2^{n v}}{u ! v !} \lesssim$ 1 , we conclude that

$$
M_{\gamma}^{1}(f, g)(x) \lesssim M f(x) M g(x) .
$$

For $r>\frac{1}{2}$, by the Hölder inequality, it leads to

$$
\left\|M_{\gamma}^{1}(f, g)\right\|_{L^{r}(\mathbb{R})} \lesssim\|f\|_{L^{p}(\mathbb{R})}\|g\|_{L^{q}(\mathbb{R})} .
$$


For $M_{\gamma}^{2}(f, g)$, as in $M_{\gamma}^{1}(f, g)$, we also have $M_{\gamma}^{2}(f, g)(x) \lesssim M f(x) M g(x)$. Therefore, for all $r>\frac{1}{2}$,

$$
\left\|M_{\gamma}^{2}(f, g)\right\|_{L^{r}(\mathbb{R})} \lesssim\|f\|_{L^{p}(\mathbb{R})}\|g\|_{L^{q}(\mathbb{R})} .
$$

For $M_{\gamma}^{3}(f, g)$, without loss of generality, we may assume that $m=n$. Therefore, we rewrite $M_{\gamma}^{3}(f, g)$ as

$$
M_{\gamma}^{3}(f, g)(x)=\sup _{j \in \mathbb{Z}}\left|\int_{-\infty}^{\infty} \int_{-\infty}^{\infty} \hat{f}(\xi) \hat{g}(\eta) e^{i \xi x} e^{i \eta x} \tilde{m}_{j}^{3}(\xi, \eta) \mathrm{d} \xi \mathrm{d} \eta\right|,
$$

where $\tilde{m}_{j}^{3}(\xi, \eta):=\sum_{m \in \mathbb{N}, k \in \mathbb{Z}} \tilde{m}_{j, m, m, k}(\xi, \eta)$. Then (1.3) can be bounded by

$$
\sum_{m \in \mathbb{N}} \sum_{j, k \in \mathbb{Z}}\left|\int_{-\infty}^{\infty} \int_{-\infty}^{\infty} \hat{f}(\xi) \hat{g}(\eta) e^{i \xi x} e^{i \eta x} \tilde{m}_{j, m, m, k}(\xi, \eta) \mathrm{d} \xi \mathrm{d} \eta\right| .
$$

As in (5.5) and (5.6), we have that there exists a positive constant $\varepsilon_{0}$ such that

$$
\left\|\sum_{j, k \in \mathbb{Z}} \mid \int_{-\infty}^{\infty} \int_{-\infty}^{\infty} \hat{f}(\xi) \hat{g}(\eta) e^{i \xi x} e^{i \eta x} \tilde{m}_{j, m, m, k}(\xi, \eta) \mathrm{d} \xi \mathrm{d} \eta\right\|\left\|_{L^{1}(\mathbb{R})} \lesssim 2^{-\varepsilon_{0} m}\right\| f\left\|_{L^{2}(\mathbb{R})}\right\| g \|_{L^{2}(\mathbb{R})}
$$

holds uniformly for $j, k \in \mathbb{Z}$. As in 6.3), we have

$$
\left\|\sum_{j, k \in \mathbb{Z}} \mid \int_{-\infty}^{\infty} \int_{-\infty}^{\infty} \hat{f}(\xi) \hat{g}(\eta) e^{i \xi x} e^{i \eta x} \tilde{m}_{j, m, m, k}(\xi, \eta) \mathrm{d} \xi \mathrm{d} \eta\right\|\left\|_{L^{r, \infty}(\mathbb{R})} \lesssim m\right\| f\left\|_{L^{p}(\mathbb{R})}\right\| g \|_{L^{q}(\mathbb{R})}
$$

holds uniformly for $j, k \in \mathbb{Z}$. By interpolating between (7.5) and (7.6), we conclude that

$$
\left\|M_{\gamma}^{3}(f, g)\right\|_{L^{r}(\mathbb{R})} \lesssim\|f\|_{L^{p}(\mathbb{R})}\|g\|_{L^{q}(\mathbb{R})}
$$

for $r>\frac{1}{2}$. From (7.1), (7.2) and (7.7), we obtain the $L^{p}(\mathbb{R}) \times L^{q}(\mathbb{R}) \rightarrow L^{r}(\mathbb{R})$ boundedness for the (sub)bilinear maximal function $M_{\gamma}(f, g)$ for $r>\frac{1}{2}$.

\section{References}

[1] P. Auscher, S. Hofmann, C. Muscalu, T. Tao and C. Thiele, Carleson measures, trees, extrapolation, and $T(b)$ theorems, Publ. Mat. 46 (2002), no. 2, 257-325.

[2] J. Bourgain, A nonlinear version of Roth's theorem for sets of positive density in the real line, J. Analyse Math. 50 (1988), 169-181.

[3] J. Bourgain, A Szemerédi type theorem for sets of positive density in $\mathbb{R}^{k}$, Israel J. Math. 54 (1986), no. 3, 307-316.

[4] A. -P. Calderón, Cauchy integrals on Lipschitz curves and ralated operators, Proc. Nat. Acad. Sci. U.S.A. 74 (1977), no. 4, 1324-1327.

[5] A. Carbery, M. Christ, J. Vance, S. Wainger and D. Watson, Operators associated to flat plane curves: $L^{p}$ estimates via dilation methods, Duke Math. J. 59 (1989), no. 3, 675-700. 
[6] A. Carbery, J. Vance, S. Wainger and D. Watson, The Hilbert transform and maximal function along flat curves, dilations, and differential equations, Amer. J. Math. 116 (1994), no. 5, 1203-1239.

[7] H. Carlsson, M. Christ, A. Cordoba, J. Duoandikoetxea, J. L. Rubio de Francia, J. Vance, S. Wainger and D. Weinberg, $L^{p}$ estimates for maximal functions and Hilbert transforms along flat convex curves in $\mathbb{R}^{2}$, Bull. Amer. Math. Soc. (N.S.) 14 (1986), no. 2, 263-267.

[8] M. Christ, X. Li, T. Tao and C. Thiele, On multilinear oscillatory integrals, nonsingular and singular, Duke Math. J. 130 (2005), no. 2, 321-351.

[9] M. Christ, A. Nagel, E. M. Stein and S. Wainger, Singular and maximal Radon transforms: analysis and geometry, Ann. of Math. 150 (1999), no. 2, 489-577.

[10] C. Demeter, Pointwise convergence of the ergodic bilinear Hilbert transform, Illinois J. Math. 51 (2007), no. 4, 1123-1158.

[11] C. Demeter and C. Thiele, On the two-dimensional bilinear Hilbert transform, Amer. J. Math. 132 (2010), no. 1, 201-256.

[12] D. Dong, Full range boundedness of bilinear Hilbert transform along certain polynomials, Math. Inequal. Appl. 22 (2019), no. 1, 151-156.

[13] D. Dong, Quasi pieces of the bilinear Hilbert transform incorporated into a paraproduct, J. Geom. Anal. 29 (2019), no. 1, 224-246.

[14] D. Dong and X. Li, On a hybrid of bilinear Hilbert transform and paraproduct, Acta Math. Sin. (Engl. Ser.) 34 (2018), no. 1, 29-41.

[15] D. Dong and X. Meng, Discrete bilinear Radon transforms along arithmetic functions with many common values, Bull. Lond. Math. Soc. 50 (2018), no. 1, 132-142.

[16] P. Durcik, S. Guo and J. Roos, A polynomial Roth theorem on the real line, Trans. Amer. Math. Soc. 371 (2019), no. 10, 6973-6993.

[17] E. B. Fabes and N. M. Rivière, Singular integrals with mixed homogeneity, Studia Math. 27 (1966), 19-38.

[18] D. Fan and X. Li, A bilinear oscillatory integral along parabolas, Positivity 13 (2009), no. 2, 339-366.

[19] H. Furstenberg, Nonconventional ergodic averages, In: The legacy of John von Neumann (Hempstead, NY, 1988), Proc. Sympos. Pure Math.,vol. 50, Amer. Math. Soc., Providence, RI, 1990.

[20] A. Gaitan and V. Lie, The boundedness of the (sub)bilinear maximal function along "nonflat" smooth curves, arXiv: 1903.11002.

[21] W. T. Gowers, A new proof of Szemerédis theorem for arithmetic progressions of length four, Geom. Funct. Anal. 8 (1998), no. 3, 529-551.

[22] L. Grafakos, Classical Fourier analysis, Third edition, Graduate Texts in Mathematics, 249. Springer, New York, 2014. xviii+638 pp. ISBN: 978-1-4939-1193-6; 978-1-4939-1194-3.

[23] L. Grafakos and X. Li, Uniform bounds for the bilinear Hilbert transforms. I, Ann. of Math. (2) 159 (2004), no. 3, 889-933.

[24] J. Guo and L. Xiao, Bilinear Hilbert transforms associated with plane curves, J. Geom. Anal. 26 (2016), no. 2, 967-995.

[25] S. Guo, L. B. Pierce, J. Roos and P. Yung, Polynomial Carleson operators along monomial curves in the plane, J. Geom. Anal. 27 (2017), no. 4, 2977-3012.

[26] L. Hörmander, Oscillatory integrals and multipliers on $F L^{p}$, Ark. Mat. 11 (1973), 1-11. 
[27] B. Host and B. Kra, Convergence of polynomial ergodic averages, Probability in mathematics, Israel J. Math. 149 (2005), 1-19.

[28] B. F. Jones, A class of singular integrals, Amer. J. Math. 86 (1964), 441-462.

[29] M. T. Lacey, The bilinear maximal functions map into $L^{p}$ for $\frac{2}{3}<p \leq 1$, Ann. of Math. (2) 151 (2000), no. 1, 35-57.

[30] M. T. Lacey and C. Thiele, $L^{p}$ estimates on the bilinear Hilbert transform for $2<p<\infty$, Ann. of Math. (2) 146 (1997), no. 3, 693-724.

[31] M. T. Lacey and C. Thiele, On Calderón's conjecture, Ann. of Math. (2) 149 (1999), no. 2, 475-496.

[32] J. Li and P. Liu, Bilinear fractional integral along homogeneous curves, J. Fourier Anal. Appl. 23 (2017), no. 6, 1465-1479.

[33] J. Li and $\mathrm{H}$. Yu, $L^{p}$ boundedness of Hilbert transforms associated with variable plane curves, arXiv: 1806.08589.

[34] X. Li, Bilinear Hilbert transforms along curves I: The monomial case, Anal. PDE 6 (2013), no. $1,197-220$.

[35] X. Li, Uniform bounds for the bilinear Hilbert transforms. II, Rev. Mat. Iberoam. 22 (2006), no. 3, 1069-1126.

[36] X. Li, Uniform estimates for some paraproducts, New York J. Math. 14 (2008), 145-192.

[37] X. Li and L. Xiao, Uniform estimates for bilinear Hilbert transforms and bilinear maximal functions associated to polynomials, Amer. J. Math. 138 (2016), no. 4, 907-962.

[38] V. Lie, A unified approach to three themes in harmonic analysis (1st part), arXiv: 1902.03807.

[39] V. Lie, On the boundedness of the bilinear Hilbert transform along "non-flat" smooth curves, Amer. J. Math. 137 (2015), no. 2, 313-363.

[40] V. Lie, On the boundedness of the bilinear Hilbert transform along "non-flat" smooth curves. The Banach triangle case $\left(L^{r}, 1 \leq r<\infty\right)$, Rev. Mat. Iberoam. 34 (2018), no. 1, 331-353.

[41] A. Nagel, J. Vance, S. Wainger and D. Weinberg, Hilbert transforms for convex curves, Duke Math. J. 50 (1983), no. 3, 735-744.

[42] E. M. Stein, Harmonic Analysis: Real-Variable Methods, Orthogonality, and Oscillatory Integrals, Princeton Mathematical Series 43, Monographs in Harmonic Analysis III, Princeton University Press, Princeton, NJ, 1993.

[43] J. Vance, S. Wainger and J. Wright, The Hilbert transform and maximal function along nonconvex curves in the plane, Rev. Mat. Iberoam. 10 (1994), no. 1, 93-121.

\section{Junfeng Li}

School of Mathematical Sciences, Dalian University of Technology, Dalian, 116024, People's Republic of China

E-mail: junfengli@dlut.edu.cn

Haixia Yu (Corresponding author)

Department of Mathematics, Sun Yat-sen University, Guangzhou, 510275, People's Republic of China

E-mail: yuhx26@mail.sysu.edu.cn 